\title{
Light groups of isometries and polyhedrality of Banach spaces
}

Leandro Antunes

THESIS PRESENTED

TO

Institute of Mathematics and Statistics

$\mathrm{OF}$

UNIVERSity OF SÃo PAUlO

IN

PARTIAL FULFILLMENT OF

THE

REQUIREMENTS FOR THE DEGREE

$\mathrm{OF}$

Doctor of ScIEnCES

Graduate Program in Mathematics

Advisor: Prof. Dr. Valentin Raphael Henri Ferenczi

This study was financed in part by Coordination for the Improvement of Higher Education Personnel (CAPES) - Finance Code 001 and by Federal University of Technology - Paraná (UTFPR) - Campus Toledo. 



\section{Light groups of isometries and polyhedrality of Banach spaces}

This version contains the corrections and changes suggested by the committee during the defense of the original version of the thesis, held in 17 June 2019. A copy of the original version is available at the Institute of Mathematics and Statistics of University of São Paulo.

Committee:

- Prof. Dr. Valentin Raphael Henri Ferenczi (advisor) - IME-USP

- Prof. Dra. Christina Brech - IME-USP

- Prof. Dr. Kevin Beanland - Washington and Lee University

- Prof. Dr. Leandro Candido Batista - UNIFESP - São José dos Campos

- Dr. Willian Hans Goes Corrêa - IME-USP 

To my family. 



\section{Acknowledgements}

I would like to express my gratitude to my advisor Valentin Ferenczi for the continuous support during my Ph.D. His guidance helped me in all this time and had a deep impact in the way I will continue my work from now on.

I also thank my advisor during my visit period in Washington and Lee University, Kevin Beanland, for presenting me many new research areas and for his many teachings. His way of doing research was very inspirational and I hope to take it as an example for myself.

I am deeply thankful to the other coauthors of the papers that generated this thesis, Sophie Grivaux, Christian Rosendal and Hùng Chu.

I thank the committee, for the corrections and suggestions for this thesis.

I would like to express my gratitude to the staff and faculty of USP, W\&L and UTFPR for all the help in the last four years.

I thank my family and friends for the patience, support and encouragement.

Finally, I thank UTFPR and CAPES for the financial support. 

Mathematics is the most beautiful and most powerful creation of the human spirit. (Stefan Banach) 



\section{Abstract}

Megrelishvili defines light groups of isomorphisms of a Banach space as the groups on which the weak and strong operator topologies coincide and proves that every bounded group of isomorphisms of Banach spaces with the point of continuity property (PCP) is light. We investigate this concept for isomorphism groups $G$ of classical Banach spaces $X$ without the PCP, especially isometry groups, and relate it to the existence of $G$-invariant LUR or strictly convex renormings of $X$. We give an example of a Banach space $X$ and an infinite countable group of isomorphisms $G \leqslant G L(X)$ which is SOT-discrete but such that $X$ does not admit a distinguished point for $G$, providing a negative answer to a question of Ferenczi and Rosendal. We also prove that every combinatorial Banach space is (V)polyhedral. In particular, the Schreier spaces of countable order provide new solutions to a problem proposed by Lindenstrauss concerning the existence of an infinite-dimensional Banach space whose unit ball is the closed convex hull of its extreme points.

Key-words: Light groups. LUR renormings. Distinguished points. Combinatorial spaces. Polyhedrality. 



\section{Resumo}

Megrelishvili define grupos leves de isomorfismos de um espaço de Banach como os grupos em que as topologias fraca e forte do operador coincidem e prova que todo grupo limitado de isomorfismos de espaços de Banach com a propriedade do ponto de continuidade (PCP) é leve. Investigamos esse conceito para grupos de isomorfismos de espaços de Banach clássicos sem PCP, especialmente grupos de isometrias, e o relacionamos com a existência de renormações $G$-invariantes LUR ou uniformemente convexas. Damos um exemplo de um espaço de Banach $X$ e um grupo enumerável infinito de isomorfismos $G \leqslant G L(X)$ que é SOT-discreto mas tal que $X$ não admite ponto distinto em relação a $G$, fornecendo uma resposta negativa a uma questão de Ferenczi e Rosendal. Também provamos que todos espaços de Banach combinatórios são (V)-poliedrais. Em particular, os espaços de Schreier de ordem enumerável fornecem novas soluções para um problema proposto por Lindenstrauss sobre a existência de um espaço de Banach de dimensão infinita cuja bola unitária seja igual a envoltória convexa fechada de seus pontos extremos.

Palavras-chave: Grupos leves. Renormações LUR. Pontos distintos. Espaços combinatórios. Poliedralidade. 



\section{List of Acronyms}

CPCP Convex point of continuity property.

CSRP Convex series representation property.

KKP Kadec-Klee Property.

LUC Locally uniformly convex.

LUR Locally uniformly rotund.

PCP Point of continuity property.

RNP Radon-Nikodym property.

SOT Strong operator topology.

WOT Weak operator topology. 



\section{List of Symbols}

$B(X) \quad$ Space of bounded linear operators $T: X \rightarrow X$, p. 25

$B(X, Y) \quad$ Space of bounded linear operators between normed spaces $X$ and $Y$, p. 25

$B_{X} \quad$ Closed unit ball of $X$, p. 25

$\operatorname{co}(F) \quad$ Convex hull of $F$, p. 71

$\overline{\mathrm{co}}(F) \quad$ Closed convex hull of $F$, p. 71

$\delta_{i, j} \quad 0$, if $i \neq j$ and 1 , if $i=j$, p. 45

$E<F \quad \max E<\min F$, p. 40

$\operatorname{ext}(C) \quad$ Set of extreme points of $C$, p. 42

$\mathcal{F} \quad$ Regular family, p. 40

$\mathcal{F}^{\text {max }} \quad$ Maximal sets of $\mathcal{F}$, p. 40

$G L(X) \quad$ Group of linear isomorphisms $T: X \rightarrow X$, p. 26

$H \quad$ Hilbert space $L_{2}[0,1]$, p. 66

$\operatorname{Isom}(X) \quad$ Group of surjective linear isometries of $X$, p. 29

$n<E \quad n<\min E$, p. 40

$\mathcal{S}_{1} \quad$ Schreier family, p. 39

$\mathcal{S}_{\alpha} \quad$ Schreier family of order $\alpha$, p. 39

$\operatorname{supp} x \quad$ Support of $x$, p. 42

$S_{X} \quad$ Unit sphere of $X$, p. 25

$W_{\mathcal{F}} \quad\left\{g=\sum_{k \in F} \pm e_{k}^{*} ; F \in \mathcal{F}\right\}$, p. 76

$W_{\mathcal{F} \max } \quad\left\{g=\sum_{k \in F} \pm e_{k}^{*} ; F \in \mathcal{F}^{\max }\right\}$, p. 80

$X_{\mathcal{F}} \quad$ Combinatorial Banach space defined by $\mathcal{F}$, p. 40

$X_{\mathcal{S}_{1}} \quad$ Schreier space, p. 39

$X_{\mathcal{S}_{\alpha}} \quad$ Schreier space of order $\alpha$, p. 40 
Extension relation, p. 77

$\stackrel{\max }{\sqsubseteq} \quad$ Maximal extension relation, p. 77 


\section{Contents}

Introduction. . . . . . . . . . . . . . . . . . . . . . . . 21

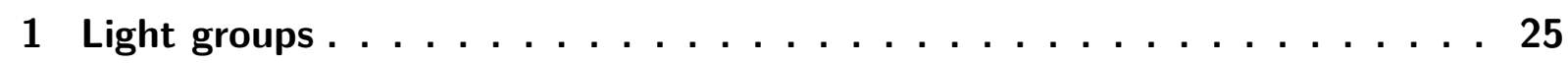

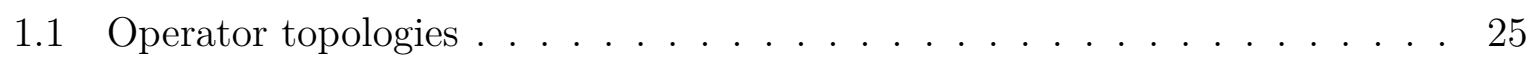

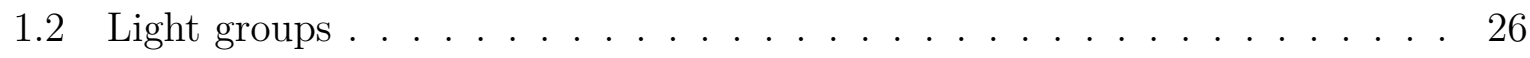

1.3 Banach spaces with PCP and RNP $\ldots \ldots \ldots \ldots \ldots \ldots \ldots \ldots$

1.4 Megrelishvili's results $\ldots \ldots \ldots \ldots \ldots$

1.5 Non-light classical Banach spaces $\ldots \ldots \ldots \ldots \ldots \ldots$

1.6 Light classical Banach spaces $\ldots \ldots \ldots \ldots . \ldots \ldots$

1.7 SOT-compact groups $\ldots \ldots \ldots \ldots \ldots \ldots \ldots \ldots \ldots$

Questions and comments . . . . . . . . . . . . . . . . 36

2 Isometries of Schreier spaces $\ldots \ldots \ldots$

2.1 Schreier spaces $\ldots \ldots \ldots \ldots \ldots$

2.2 Decompositions and spreads of Schreier sets $\ldots \ldots \ldots \ldots \ldots$

2.3 Isometry groups of Schreier spaces. . . . . . . . . . . . . . . . 42

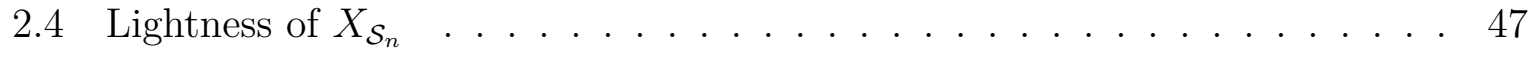

Questions and comments . . . . . . . . . . . . . . . . 47

3 Convexity of norms and light groups . . . . . . . . . . . 49

3.1 Convexity of norms in vector spaces $\ldots \ldots \ldots \ldots \ldots$

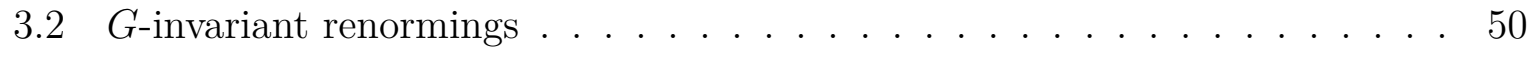

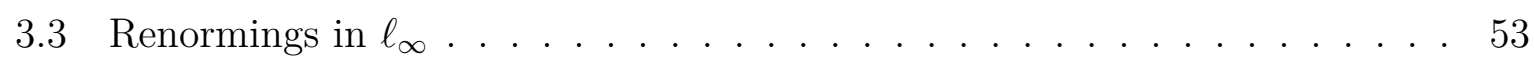

$3.4 \quad$ A light space without isometry invariant LUR renormings . . . . . . . . . 54

Questions and comments . . . . . . . . . . . . . . . 58

4 Distinguished points and light groups . . . . . . . . . . . . 59

4.1 Distinguished family for dual actions $\ldots \ldots \ldots \ldots \ldots \ldots$

4.2 Non-light renormings of $C(K) \ldots \ldots \ldots \ldots \ldots \ldots \ldots$

4.3 Example of an SOT-discrete group without distinguished points . . . . . 64

4.4 SOT-discrete groups with dense orbit $\ldots \ldots \ldots \ldots \ldots$

Questions and comments . . . . . . . . . . . . . . . 6 68

5 Polyhedrality of combinatorial spaces $\ldots \ldots \ldots \ldots \ldots \ldots \ldots$

$5.1 \quad \lambda$-property and CSRP $\ldots \ldots \ldots \ldots \ldots \ldots$

5.2 Characterization of the dual of combinatorial spaces . . . . . . . . 74

5.3 Polyhedrality of combinatorial spaces $\ldots \ldots \ldots \ldots \ldots \ldots$

Questions and comments . . . . . . . . . . . . . . . . . 83

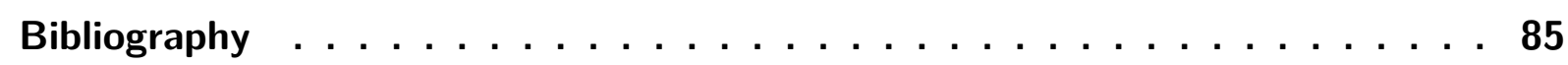

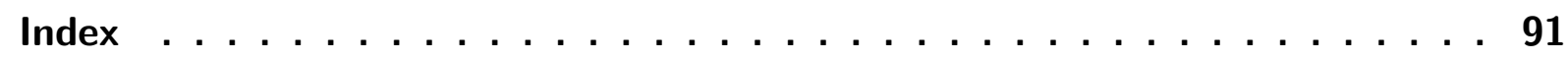





\section{Introduction}

The purpose of this thesis is to investigate geometric properties of Banach spaces, especially light groups of isomorphisms, convexity of norms, distinguished points for group actions and polyhedrality. Chapters 1,3 and 4 are part of a joint work with Valentin Ferenczi, Sophie Grivaux and Christian Rosendal [AFGR17]. The results of Chapters 2 and 5 are part of a joint work with Kevin Beanland and Hùng Chu [ABC19, obtained during the author's doctoral exchange program (Programa de Doutorado Sanduíche no Exterior - PDSE-CAPES) in Washington and Lee University, under the guidance of Prof. Kevin Beanland.

A frequent problem in functional analysis is to determine under which conditions weak convergence and norm convergence coincide. For example, it is well-known that conditions of convexity of the norm of a Banach space ensure that weak and strong convergence are equivalent on its unit sphere. The corresponding problem for isomorphisms of Banach spaces (or more generally of locally convex spaces) was studied by Megrelishvili in Meg01. He calls a bounded group of isomorphisms of a Banach space $G \leqslant \mathrm{GL}(X)$ light if the Weak Operator Topology (WOT) and the Strong Operator Topology (SOT) coincide on $G$.

Megrelishvili proves that if $X$ is a Banach space with the Point of Continuity Property (PCP) and if $G \leqslant G L(X)$ is bounded in norm, then $G$ is light. In particular, if $X$ is reflexive or if it is a separable dual space, then every bounded subgroup of $G L(X)$ is light. For example, the isometry groups of $\ell_{1}$ and $L_{p}[0,1]$, for $1<p<\infty$, are light.

A natural question that arises from Megrelishvili's result is in which respect it is optimal and whether "smallness" assumptions on $G$ or weaker assumptions than the PCP on $X$ could imply that $G$ is light. To investigate this question, we classify the isomorphism groups (especially the isometry groups) of classical Banach spaces in terms of being light. In Chapter 1 we classify the isometry groups of $\ell_{\infty}, L_{1}[0,1]$ and $C(K)$. We also prove that every SOT-compact group is light.

In Chapter 2 we classify the isometry group of Schreier spaces of finite order. This problem motivated the characterization of the isometry group of these spaces, that was obtained later by Kevin Beanland and Hùng Chu using properties of extreme points of Schreier spaces. We also reproduce their proof, with minor modifications.

We start to connect the concept of light groups with other geometric properties of Banach spaces in Chapter 3. We study the relation between light groups and the existence of locally uniformly rotund (LUR) renormings invariant under the action of the group. 
Kadec proved in Kad59 that every separable Banach space admits an LUR renorming. If, in addition, $X$ has the Radon-Nikodym property (RNP), Lancien showed in [Lan93] that this LUR renorming can be taken in such a manner that it preserves the original isometries of $X$. In this case, we say that $X$ admits an isometry invariant LUR renorming. In general, if $G \leqslant \mathrm{GL}(X,\|\cdot\|)$ is a bounded group of isomorphisms on $X$, the norm on $X$ defined by

$$
\|x\|=\sup _{g \in G}\|g x\|, x \in X,
$$

is a $G$-invariant renorming of $X$. In other words, $G \leqslant \operatorname{Isom}(X,\|\cdot\|)$. So a consequence of Lancien's Theorem is that whenever $X$ is a separable space with the RNP and $G$ is a bounded group of isomorphisms on $X$, there exists a $G$-invariant LUR renorming of $X$.

In Theorem 3.3 we prove that if a Banach space $X$ admits a $G$-invariant LUR renorming, then $G$ is light. In fact, this is true even if the norm is LUR only on a dense subset of $S_{X}$. We also show that the converse assertion is false: although the isometry group of $C[0,1]$ is light, $C[0,1]$ admits no strictly convex isometry invariant renorming (Corollary 3.11). In particular, Lancien's result cannot be generalized to every separable Banach space. Another consequence of Theorem 3.3 is that the isometry group of $c_{0}$ is light, due to the existence of an isometry invariant LUR renorming of $c_{0}$ provided by Day in Day55].

In Chapter 4 we will connect the concepts of light groups with the concept of distinguished point, defined by Ferenczi and Rosendal in [FR11]. Ferenczi and Galego investigated in [FG10] groups that may be seen as the group of isometries of a Banach space under some renorming. Among other results, they prove that if $X$ is a separable Banach space with LUR norm $\|\cdot\|$ and if $G$ is an infinite countable isometry group of $X$ such that $-\operatorname{Id} \in G$ and such that $G$ admits a point $x \in X$ with $\inf _{g \neq \mathrm{Id}}\|g x-x\|>0$, then $G=\operatorname{Isom}(X,\|\cdot\|)$ for some equivalent norm $\|\cdot\|$ on $X$. A point $x$ satisfying the condition

$$
\inf _{g \neq \mathrm{Id}}\|g x-x\|>0
$$

is called in [FR11] a distinguished point of $X$ for the group $G$. In Theorem 4.4 we prove that if $G \leqslant G L(X)$ is such that $G$ has a distinguished point for $X$, but $G$ does not act as an SOT-discrete group on $X^{*}$, then $G$ is not light. In particular, the isometry group of $c$ is not light (Corollary 4.7). A consequence of this fact is that every infinite dimensional separable $C(K)$ admits a non light renorming (Corollary 4.9), i.e., a renorming such that the corresponding isometry group is not light.

It is clear that if $G$ is an isometry group with a distinguished point then $G$ is SOT-discrete. Ferenczi and Rosendal proposed the following question in [FR11]: if $G$ is an isomorphism group of $X$ which is SOT-discrete, should $X$ have a distinguished point for $G$ ? We give in Proposition 4.11 an example of a group $G \leqslant G L\left(c_{0}\right)$ that provide a negative answer to this question. In addition, this group is not light. 
In Chapter 5 we return to the study of Schreier spaces, initiated in Chapter 2. We provide a characterization for the extreme points of the dual of Schreier spaces of countable order (Proposition 1.10). In fact, this result holds for any combinatorial space, i.e., for any sequence space in which the norm is defined by a compact, hereditary and spreading family of finite sets of $\mathbb{N}$.

We will also prove that the Schreier spaces of countable order provide new solutions for a problem of Lindenstrauss [Lin66] concerning polyhedrality. A Banach space $X$ is called polyhedral if the unit ball of every finite dimensional subspace of $X$ is a polytope (i.e. has finitely many extreme points). Some examples of polyhedral spaces are $c_{0}$ and $C(K)$ spaces for $K$ a countable, compact, Hausdorff space.

Lindenstrauss proved in [Lin66] that every infinite-dimensional space has a twodimensional quotient space whose unit ball is not a polygon. In particular, no infinitedimensional dual space (including reflexive spaces) is polyhedral. A consequence of KreinMilman Theorem is that if $X$ is reflexive, then

$$
B_{X}=\overline{\mathrm{co}}\left(\operatorname{ext} B_{X}\right)
$$

This led Lindenstrauss to ask whether there exist a polyhedral infinite-dimensional Banach space whose unit ball is the closed convex hull of its extreme points. The first solution to this problem was provided by De Bernardi in [DB17], using a renorming of $c_{0}$. In fact, De Bernardi proves that $c_{0}$ with this renorming satisfies a stronger condition, called (V)-polyhedrality.

The unit ball of Schreier spaces of countable order are the closed convex hull of their sets of extreme points, since they have the convex series representation property (CSRP). In Theorem 5.12 we will prove that these spaces are also (V)-polyhedral and hence provide new answers to Lindenstrauss's problem. We also prove (Corollary 5.10) that the dual space of every combinatorial space has the CSRP, i.e., every point of the unit ball of $X_{\mathcal{F}}^{*}$ can be expressed as an infinite convex combination of extreme points of $X_{\mathcal{F}}^{*}$.

We summarize the classification of groups in terms of being light cited in this thesis in page 69 .

In this thesis all Banach spaces are assumed to be real spaces and all isometries are assumed to be linear and surjective. 



\section{Light groups}

A frequent problem in functional analysis is to determine when a weak convergence implies a strong convergence. Megrelishvili studied this problem in Meg01 focusing in the continuity of group representations in locally convex spaces, especially in Fréchet spaces and Banach spaces. For this reason, he considered bounded groups of isomorphisms $T: X \rightarrow X$ in which the weak operator topology and the strong operator topologies coincide. These groups are called light.

\subsection{Operator topologies}

Let $X$ and $Y$ be normed spaces and let $B(X, Y)$ be the space of bounded linear operators $T: X \rightarrow Y$. When $X=Y$ we will denote $B(X, X)$ simply by $B(X)$. The closed unit ball of $X,\{x \in X:\|x\| \leqslant 1\}$ will be denoted by $B_{X}$ and the unit sphere of $X$, $\{x \in X:\|x\|=1\}$ will be denoted by $S_{X}$.

Recall that

$$
\|T\|=\sup _{x \in B_{X}}\|T x\|
$$

defines a norm in $B(X, Y)$ and this norm is complete if $Y$ is a Banach space. Thus, we can consider the norm topology in $B(X, Y)$ defined by this norm. However, we will be interested in this chapter in weaker topologies in $B(X, Y)$.

The strong operator topology (SOT) in $B(X, Y)$ is the topology generated by the family of seminorms

$$
\begin{aligned}
f_{x}: B(X, Y) & \rightarrow Y \\
T & \mapsto T x,
\end{aligned}
$$

with $x \in X$. In other words, SOT is the topology of pointwise convergence. The weak operator topology (WOT) in $B(X, Y)$ is the topology generated by the family of seminorms

$$
\begin{aligned}
f_{x, y^{*}}: B(X, Y) & \rightarrow \mathbb{K} \\
T & \mapsto y^{*} T x,
\end{aligned}
$$

with $x \in X$ and $y^{*} \in Y^{*}$. In other words, the WOT is the topology of pointwise convergence in $B(X, Y)$, when $Y$ is equipped with the weak topology.

A useful way to characterize these topologies is using nets (for definitions and properties of nets, see Meg98, Chapter 2]). A net $\left(T_{\alpha}\right)_{\alpha \in I} \subset B(X, Y)$ converges to $T$ in SOT if, and only if, for every $\varepsilon>0$ and every $x \in X$ there exists $\alpha_{0} \in I$ such that

$$
\alpha \succcurlyeq \alpha_{0} \Longrightarrow\left\|T_{\alpha} x-T x\right\|<\varepsilon .
$$


In the same way, a net $\left(T_{\alpha}\right)_{\alpha \in I} \subset B(X, Y)$ converges to $T$ in WOT if, and only if, for every $\varepsilon>0$, every $x \in X$ and every $y^{*} \in Y^{*}$ there exists $\alpha_{0} \in I$ such that

$$
\alpha \succcurlyeq \alpha_{0} \Longrightarrow\left|y^{*} T_{\alpha} x-y^{*} T x\right|<\varepsilon \text {. }
$$

Since the inequality

$$
\left|y^{*} T x-y^{*} S x\right| \leqslant\left\|y^{*}\right\| \cdot\|T x-S x\| \leqslant\left\|y^{*}\right\| \cdot\|T-S\| \cdot\|x\|,
$$

holds for every $x \in X, y^{*} \in Y^{*}$ and $S, T \in B(X, Y)$, we can conclude that WOT is indeed a weaker topology than SOT and that SOT is weaker than the norm topology.

\subsection{Light groups}

Let $X$ be a locally convex space. Megrelishvili calls a group of isomorphisms $G \leqslant$ $G L(X)$ light in Meg01 if the weak and the strong operator topologies coincide on $G$ (a set $G \subset G L(X)$ with this property is called a Kadec subset by Glasner and Megrelishvili in GM14]).

We will be interested in bounded groups of isomorphisms, i.e., groups $G \leqslant G L(X)$ such that

$$
\sup _{T \in G}\|T\|<\infty
$$

Example 1.1. Let $H$ be a Hilbert space. We say that an operator $T: H \rightarrow H$ is unitary if

$$
T^{*} T=\mathrm{Id}=T T^{*}
$$

where $T^{*}$ is the adjoint operator of $T$. Notice that every unitary operator $T$ is an isometry in $H$, since for every $x \in H$ we have

$$
\|T x\|^{2}=\langle T x, T x\rangle=\left\langle x, T^{*} T x\right\rangle=\langle x, x\rangle=\|x\|^{2} .
$$

The set of unitary operators on $H$ defines a group, called unitary group of $H$ and denoted by $U(H)$.

We claim that $U(H)$ is light. Notice that it suffices to show that for every net $\left(T_{\alpha}\right)_{\alpha \in I}$ in $U(H)$ such that $T_{\alpha} \stackrel{\text { WOT }}{\longrightarrow}$ Id we have $T_{\alpha} \stackrel{\text { SOT }}{\longrightarrow}$ Id.

Let $\left(T_{\alpha}\right)_{\alpha \in I}$ in $U(H)$ such that $T_{\alpha} \stackrel{\text { WOT }}{\longrightarrow}$ Id. For every $x$ and $y \in H$ we have

$$
\left\langle T_{\alpha} x, y\right\rangle \rightarrow\langle x, y\rangle \quad \text { and } \quad\left\langle y, T_{\alpha} x\right\rangle \rightarrow\langle y, x\rangle .
$$

Hence, for every $x \in H$ we have:

$$
\left\|T_{\alpha} x-x\right\|^{2}=\left\langle T_{\alpha} x-x, T_{\alpha} x-x\right\rangle=\left\langle T_{\alpha} x, T_{\alpha} x\right\rangle-\left\langle x, T_{\alpha} x\right\rangle-\left\langle T_{\alpha} x, x\right\rangle+\langle x, x\rangle \rightarrow 0,
$$

i.e., $T_{\alpha} \stackrel{\text { SOT }}{\longrightarrow} \mathrm{Id}$. 
Example 1.2. A locally compact group $G$ is called amenable if it admits a left invariant mean on $L^{\infty}(G)$, i.e., there exists a Borel measure $\mu$ with $\mu(G)=1$ and $\mu(g A)=\mu(A)$ for every $g \in G$ and $A \subseteq G$ measurable. It is a well-know fact that every uniformly bounded continuous representation of an amenable group on a Hilbert $H$ space is equivalent to a continuous unitary representation (see [Pie84, Corollary 17.6]). Hence, every bounded representation of an amenable group on $H$ has light image.

In fact, Megrelishvili proves that every bounded group (amenable or not) $G \leqslant$ $G L(X)$ of a Banach space with the Point of Continuity Property is light.

\subsection{Banach spaces with PCP and RNP}

Let $C$ be a norm-compact subset of a Banach space $X$. It is easy to see that the weak topology $\tau_{w}$ and the norm topology $\tau_{\|\cdot\|}$ coincide on $C$. However, if $C$ is only weakly compact, the identity map $\left(C, \tau_{w}\right) \rightarrow\left(C, \tau_{\|\cdot\|}\right)$ is not continuous, in general. Namioka studied in [Nam67] how the points of continuity for this map were distributed in $C$, in particular if the extreme points of $C$ are points of continuity.

In [Bou80] Bourgain says that a Banach space has the property (*) if for each nonempty, bounded, closed and convex subset $C \subset X$, the identity map Id $:\left(C, \tau_{w}\right) \rightarrow\left(C, \tau_{\|\cdot\|}\right)$ has a point of continuity. This property is currently called convex point of continuity property $(C P C P)$. If Id $:\left(C, \tau_{w}\right) \rightarrow\left(C, \tau_{\|\cdot\|}\right)$ has a point of continuity for every non-empty, bounded and closed subset $C \subset X$, we say that $X$ has the point of continuity property $(P C P)$.

Remark 1.3. Sometimes in literature the condition "closed" in the definition of PCP is replaced by "weakly-closed" (cf. [Pie07, p. 229] and [FLP01, p. 636]). However, both definitions are equivalent. Indeed, let's denote by PCP' the Banach spaces that satisfy this second definition. It is immediate that PCP $\Longrightarrow \mathrm{PCP}^{\prime}$, since every weakly closed subset of $X$ is norm closed. On the other hand, suppose that $X$ has the PCP' and let $F$ be a norm closed, bounded and non-empty subset of $X$. Let $G$ be the closure of $F$ in the weak topology. Then, there exists a point of continuity $x_{0} \in G$ for the identity map Id $:\left(G, \tau_{w}\right) \rightarrow\left(G, \tau_{\|\cdot\|}\right)$. Since $x_{0}$ is a limit point of $F$ in the weak topology, there exists a net $\left(x_{\alpha}\right)_{\alpha \in I}$ in $F$ such that

$$
x_{\alpha} \stackrel{\tau_{w}}{\longrightarrow} x_{0} .
$$

By the weak-to-norm continuity of Id in $x_{0}$,

$$
x_{\alpha} \stackrel{\tau_{\|\cdot\|}}{\longrightarrow} x_{0}
$$

and, since $F$ is norm closed we have $x_{0} \in F$. Therefore $x_{0}$ is a point of continuity of Id $:\left(F, \tau_{w}\right) \rightarrow\left(F, \tau_{\|\cdot\|}\right)$ and $X$ has the PCP. 
Remark 1.4. The PCP is a hereditary property, i.e., if $X$ is a Banach space with the PCP and $Y$ is a closed subspace of $X$, then $Y$ has the PCP. Indeed, if $C$ is a non-empty, bounded and closed subset of $Y$, then $C$ is a non-empty, bounded and closed subset of $X$. Since the weak topology of $X, \tau_{w_{X}}$, restricted to $Y$ coincides with the weak topology of $Y, \tau_{w_{Y}}$, and since the norm topology of $X, \tau_{\|\cdot\|_{X}}$, restricted to $Y$ coincides with the norm topology of $Y, \tau_{\|\cdot\|_{Y}}$, then the existence of a point of continuity for

$$
\text { Id }:\left(C, \tau_{w_{X}}\right) \rightarrow\left(C, \tau_{\|\cdot\|_{X}}\right)
$$

is equivalent to the existence of a point of continuity for

$$
\mathrm{Id}:\left(C, \tau_{w_{Y}}\right) \rightarrow\left(C, \tau_{\|\cdot\|_{Y}}\right) \text {. }
$$

Examples of Banach spaces without PCP are $c_{0}$ and $L_{1}[0,1]$ (see [EW84, Table 1]). By Remark 1.4, any space that contains a copy of $c_{0}$ also fails the PCP, for example $C(K)$ for any infinite, compact and metrizable set $K$ (see [ $\mathrm{FHH}^{+} 11$, p. 273, Exercise 5.26]).

Bourgain proves in Bou80 that every space with the Radon-Nikodym property $(R N P)$ has the PCP. A Banach space has the Radon-Nikodym property if a version of the Radon-Nikodym Theorem holds for $X$. More precisely, a Banach space $X$ has the RNP with respect to a space of finite measure $(\Omega, \Sigma, \mu)$ if for every vector measure continuous with respect to $\mu$ with bounded variation $G: \Sigma \rightarrow X$ there exists $g \in L_{1}(X, \mu)$ such that

$$
G(E)=\int_{E} g d \mu
$$

for every $E \in \Sigma$. If $X$ has the RNP with respect to every finite measure space, we simply say that $X$ has the RNP. This definition was given by Chatterji in [Cha68]. See [DUJ77, p. 218] for other equivalent definitions of RNP.

Diestel [DUJ77, p. 218] gives as examples of Banach spaces with the RNP:

1. Reflexive spaces;

2. Separable dual spaces;

3. Weakly compactly generated (WCG) spaces;

4. Duals of WCG spaces;

5. Locally Uniformly Convex (LUC) spaces;

6. Dual spaces with differentiable Fréchet norm;

7. Spaces with a boundedly complete basis;

8. $\ell_{1}(\Gamma)$, for every set $\Gamma$. 


\subsection{Megrelishvili's results}

The main result of [Meg01 is the following:

Theorem 1.5 (Megrelishvili). If $X$ is a Banach space with the $P C P$ and $G \leqslant G L(X)$ is bounded, then $G$ is light.

The proof of this theorem involves the concept of fragmentability. We say that a subset $A$ of a Banach space $X$ is fragmented if for every $B \subset A$ and every $\varepsilon>0$ there exists a weakly open set $W \subset X$ such that $B \cap W \neq \emptyset$ and $\operatorname{diam}(B \cap W)<\varepsilon$. We say that $X$ is bound-fragmented if every bounded subset of $X$ is fragmented.

Megrelishvili proves that if $G \leqslant G L(X), \pi: G \times X \rightarrow X$ is a group action and $x \in X$ is such that:

1. For every $T \in G$ there exists a neighborhood $U$ of $T$ such that $U^{-1}$ is uniformly equicontinuous;

2. There exists a neighborhood $V$ of Id such that $V x$ is a fragmented subset of $X$;

3. The orbit of $x$ under the action of $G$

$$
\begin{aligned}
\tilde{x}: G & \rightarrow X \\
g & \mapsto g x
\end{aligned}
$$

is continuous, if $G$ is equipped with the WOT;

then $\tilde{x}$ is continuous with $G$ equipped with the SOT. The result then follows from the fact that a Banach space $X$ has the PCP if, and only if, it is bound-fragmented (see [JR85]).

Notice that, by Theorem 1.5, every bounded group of isomorphisms of reflexive spaces (including Hilbert spaces) or separable dual spaces is light. The objective of this chapter is to investigate the following question:

Question 1.6. Can Theorem 1.5 be improved? In other words, can we replace the condition of $X$ has PCP by a weaker one?

For this purpose, we will classify in terms of being light several bounded groups of linear isomorphisms of Banach spaces without the PCP, focusing on isometry groups of classical sequence spaces and function spaces. Since we are primarily interested in surjective linear isometry groups, we define:

Definition 1.7. A Banach space $X$ is light if its isometry group

$$
\operatorname{Isom}(X)=\{T \in G L(X):\|T x\|=\|x\|, \forall x \in X\}
$$

is light. 
Recall that if $K$ is a non-empty compact Hausdorff space, $C(K)$ denotes the space of every continuous function $f: K \rightarrow \mathbb{K}$ and $C(K)$ is a Banach space with the norm

$$
\|f\|_{\infty}=\max \{|f(x)| ; x \in K\}
$$

Megrelishvili gives in Meg01 as an example of a non-light bounded group the following:

Proposition 1.8 (Megrelishvili). The group $\operatorname{Isom}\left(C[-1,1]^{2}\right)$ is not light.

Megrelishvili uses in the proof of this proposition a Helmer construction [Hel80] of a group action on $[-1,1]^{2}$ that is separately continuous, but not jointly continuous and the equivalence between pointwise compacity and weak compacity on bounded subsets of $C(K)$. Using the same construction, we can prove that $C(K)$ is non-light, for every infinite, compact and connected subset $K \subset \mathbb{R}^{n}$ that contains a $k$-dimensional region, with $k \geqslant 2$. This raises the new question:

Question 1.9. For every compact set $K$ (Hausdorff, separable), is $C(K)$ non-light? Is $C[0,1]$ non-light?

We will give the answer of this question in Proposition 1.15.

\subsection{Non-light classical Banach spaces}

In this section we will give some examples of classical Banach spaces that are not light, namely, $\ell_{\infty}, L_{1}[0,1]$ and $C\left(\{0,1\}^{\mathbb{N}}\right)$. The proofs consist in concrete examples of sequences of isometries of these spaces that converge in WOT but not in SOT. Thus, our examples are simpler than the one provided by Megrelishvili in Meg01.

Proposition 1.10. The space $\ell_{\infty}$ is not light.

Proof. Consider the sequence $\left(T_{n}\right)$ in $\operatorname{Isom}\left(\ell_{\infty}\right)$ defined by

$$
T_{n}\left(x_{1}, \ldots, x_{n-1}, x_{n}, x_{n+1}, \ldots\right)=\left(x_{1}, \ldots, x_{n-1},-x_{n}, x_{n+1}, \ldots\right) \text {. }
$$

Obviously $T_{n} \stackrel{\text { SOT }}{\longrightarrow} \mathrm{Id}$, since

$$
\left\|T_{n}(1,1, \ldots)-(1,1, \ldots)\right\|_{\infty}=2
$$

for every $n$. On the other hand, $T_{n} \stackrel{\text { WOT }}{\longrightarrow}$ Id. Indeed, if this was not the case, there would exist $\Phi \in \ell_{\infty}^{*}, x \in \ell_{\infty}, \varepsilon>0$ and infinite indices $n_{1}, n_{2}, \ldots, n_{k}, \ldots$ such that

$$
\left|\Phi\left(T_{n_{k}}(x)\right)-\Phi(x)\right|>\varepsilon
$$


for every $k$. For each $k \in \mathbb{N}$ define $\lambda_{k}$ by

$$
\lambda_{k}=\frac{\left|\Phi\left(T_{n_{k}}(x)-x\right)\right|}{2\|x\|_{\infty} \Phi\left(T_{n_{k}}(x)-x\right)},
$$

and for each $N \in \mathbb{N}$ define $x_{N}$ by

$$
x_{N}=\sum_{k=1}^{N} \lambda_{k}\left(T_{n_{k}}(x)-x\right) .
$$

Notice that for every $N$ we have $\left\|x_{N}\right\|_{\infty} \leqslant 1$. Indeed, the coordinates of $x_{N}=$ $\left(x_{N}(1), x_{N}(2), \ldots\right)$ are given by

$$
x_{N}(k)=\left\{\begin{array}{ll}
0, & \text { if } k \neq n_{1}, n_{2}, \ldots \\
-2 \lambda_{i} x_{i}, & \text { if } k=n_{i}
\end{array} .\right.
$$

Hence,

$$
\|x\|_{\infty} \leqslant \max _{1 \leqslant i \leqslant N} 2\left|\lambda_{i} x_{i}\right|=\frac{\left|x_{i}\right|}{\|x\|_{\infty}} \leqslant 1
$$

On the other hand,

$$
\Phi\left(x_{N}\right)=\sum_{k=1}^{N} \frac{\left|\Phi\left(T_{n_{k}}(x)-x\right)\right|}{2\|x\|_{\infty} \Phi\left(T_{n_{k}}(x)-x\right)} \Phi\left(T_{n_{k}}(x)-x\right)>\frac{N \varepsilon}{2\|x\|_{\infty}},
$$

for every $N \in \mathbb{N}$, which contradicts $\Phi \in \ell_{\infty}^{*}$. Therefore, $T_{n} \stackrel{\text { WOT }}{\longrightarrow} \operatorname{Id}$ and $\operatorname{Isom}\left(\ell_{\infty}\right)$ is not light.

A similar proof of Proposition 1.10 can be used to show that the space $C\left(\{0,1\}^{\mathbb{N}}\right)$ is not light:

Proposition 1.11. The space $C\left(\{0,1\}^{\mathbb{N}}\right)$ is not light.

Proof. For each integer $n \geqslant 1$, let $N_{n}$ be the basic open set of $\{0,1\}^{\mathbb{N}}$ defined by

$$
N_{n}=\left\{\left(x_{1}, x_{2}, \ldots\right) \in\{0,1\}^{\mathbb{N}} ; \quad x_{1}=\cdots=x_{n-1}=1, \quad x_{n}=0\right\} .
$$

Define the sequence $\left(T_{n}\right)$ in $\operatorname{Isom}\left(C\left(\{0,1\}^{\mathbb{N}}\right)\right)$ by

$$
T_{n}(f)(x)=\left\{\begin{array}{l}
-f(x), \text { if } x \in N_{n}, \\
f(x), \text { otherwise. }
\end{array}\right.
$$

Then, $T_{n} \stackrel{\text { SOT }}{\longrightarrow}$ Id, since taking $f \equiv 1$ we have $\left\|T_{n}(f)-f\right\|_{\infty}=2$ for every $n$. On the other hand, the same proof of Proposition 1.10 shows that $T_{n} \stackrel{\text { WOT }}{\longrightarrow}$ Id.

It is worth noticing that the spaces $\ell_{p}$, with $1 \leqslant p<\infty$, are light by Theorem 1.5 . since they are separable dual spaces and hence have the RNP. Although $\ell_{1}$ is light, the same does not occur to $L_{1}[0,1]$. 
Remark 1.12. $L_{1}[0,1]$ is not isometrically isomorphic to any dual space, since it has no extreme points Meg98, Example 2.10.11]. Moreover, $L_{1}[0,1]$ does not have the PCP (see [EW84]).

Proposition 1.13. The space $L_{1}[0,1]$ is not light.

Proof. For every $n \in \mathbb{N}, n \geqslant 1$ define $\varphi_{n}:[0,1] \rightarrow[0,1]$ by

$$
\varphi_{n}(x)=x+\frac{1-\cos \left(2^{n} \pi x\right)}{2^{n} \pi} .
$$

Notice that $\varphi_{n}$ is a differentiable bijection, with $\varphi_{n}^{\prime} \geqslant 0$. Hence, the linear operator $T_{n}: L_{1}[0,1] \rightarrow L_{1}[0,1]$ defined by

$$
T_{n}(f)(x)=\varphi_{n}^{\prime}(x) f\left(\varphi_{n}(x)\right)
$$

is an isometry, since

$$
\left\|T_{n}(f)\right\|_{1}=\int_{0}^{1}\left|\varphi_{n}^{\prime}(x) f\left(\varphi_{n}(x)\right)\right| d x=\int_{0}^{1}|f(x)| d x=\|f\|_{1},
$$

for every $f \in L_{1}[0,1]$ and it is onto. Moreover, $T_{n} \stackrel{\text { SOT }}{\rightarrow}$ Id because taking $f \equiv 1$ we have

$$
\left\|T_{n}(1)-1\right\|_{1}=\left\|\sin \left(2^{n} \pi x\right)\right\|_{1}=\frac{2}{\pi}>0
$$

for every $n$.

We claim that

$$
\int_{0}^{1} T_{n}(f)(x) g(x) d x \rightarrow \int_{0}^{1} f(x) g(x) d x
$$

for every $f \in L_{1}[0,1]$ and $g \in L_{\infty}[0,1]$ (i.e., $T_{n} \stackrel{\text { WOT }}{\longrightarrow}$ Id). Indeed, it is well-known that the sequence $\sin (\pi n x)$ converges weakly to zero in $L_{1}[0,1]$ (Riemann-Lebesgue Lemma - see [Rud87, p. 103]). Hence, the claim holds for constant functions $f \equiv c$, since

$$
\int_{0}^{1} c\left(1+\sin \left(2^{n} \pi x\right)\right) g(x) d x \rightarrow \int_{0}^{1} c g(x) d x
$$

for every $g \in L_{\infty}[0,1]$. Consider now $f$ as the characteristic function

$$
\chi_{\left[\frac{2 k}{2^{m}}, \frac{2(k+1)}{2^{m}}\right]} \text {, with } m \geqslant 1 \text { and } 0 \leqslant k \leqslant 2^{m-1}-1 .
$$

Since $\varphi_{m}:\left[\frac{2 k}{2^{m}}, \frac{2(k+1)}{2^{m}}\right] \rightarrow\left[\frac{2 k}{2^{m}}, \frac{2(k+1)}{2^{m}}\right]$ is a bijection, then

$$
\chi_{\left[\frac{2 k}{2^{m}}, \frac{2(k+1)}{2^{m}}\right]}\left(\varphi_{m}(x)\right)=\chi_{\left[\frac{2 k}{2^{m}}, \frac{2(k+1)}{2^{m}}\right]}(x) .
$$


Therefore,

$$
\begin{aligned}
\int_{0}^{1} T_{n}\left(\chi_{\left[\frac{2 k}{2^{m}}, \frac{2(k+1)}{2^{m}}\right]}\right)(x) g(x) d x & =\int_{0}^{1} \varphi_{n}^{\prime}(x) \chi_{\left[\frac{2 k}{2^{m}}, \frac{2(k+1)}{2^{m}}\right]}\left(\varphi_{n}(x)\right) g(x) d x \\
& =\int_{0}^{1} \varphi_{n}^{\prime}(x) \chi_{\left[\frac{2 k}{2^{m}}, \frac{2(k+1)}{2^{m}}\right]}(x) g(x) d x \\
& =\int_{0}^{1} \chi_{\left[\frac{2 k}{2^{m}}, \frac{2(k+1)}{2^{m}}\right]}(x) g(x) d x+ \\
& +\int_{0}^{1} \sin \left(2^{n} \pi x\right) \chi_{\left[\frac{2 k}{2^{m}}, \frac{2(k+1)}{2^{m}}\right]}(x) g(x) d x .
\end{aligned}
$$

and by the weak convergence of $\sin \left(2^{n} \pi x\right)$ to 0 in $L_{1}[0,1]$, it follows that

$$
\int_{0}^{1} T_{n}\left(\chi_{\left[\frac{2 k}{2^{m}}, \frac{2(k+1)}{2^{m}}\right]}\right)(x) g(x) d x \rightarrow \int_{0}^{1} \chi_{\left[\frac{2 k}{2^{m}}, \frac{2(k+1)}{2^{m}}\right]}(x) g(x) d x .
$$

The general case follows from the linearity of $T_{n}$ and by density of staircase functions in $L_{1}[0,1]$.

\subsection{Light classical Banach spaces}

Recall that Megrelishvili proved that $C[-1,1]^{2}$ is not a light space. In fact, his proof can be generalized to show that $C(K)$ is not light, if $K$ is a connected compact subset of $\mathbb{R}^{n}$, with $n \geqslant 2$. However, as we will see in next proposition, this is not the case for $n=1$.

In the examples that we provided in last section, it was enough to find a sequence of isometries that converge in WOT but not in SOT to prove that those spaces were not light. However, to show that a space is light we need to verify that every net $\left(T_{\alpha}\right)$ that converges (without loss of generality, to Id) in WOT converges also in SOT. Hence, we need a characterization of the isometries of these spaces.

Fortunately, the characterizations of isometries of many classical Banach spaces can be found already in Banach's book [Ban32], although some proofs are not given completely and the theorems are stated in less than full generality. For example, the isometries of $C(K)$ were characterized by Banach for $K$ compact metric space. Stone generalized Banach's proof for compact Hausdorff spaces. Other generalizations can be found in [FJ03].

Theorem 1.14 (Banach-Stone). If $K$ and $Q$ are compact Hausdorff spaces and $T$ is an isometric isomorphism of $C(Q, \mathbb{R})$ onto $C(K, \mathbb{R})$, then there is a homeomorphism $\varphi$ from $K$ onto $Q$ and a continuous function $h: K \rightarrow \mathbb{R}$, with $|h(x)|=1$ for every $x \in K$ and such that for each $f \in C(Q)$,

$$
T f(t)=h(t) f(\varphi(t)), \text { for } t \in K \text {. }
$$


Proof. See [FJ03], Theorem 2.1.1.

Using Banach-Stone Theorem we can now prove that $C[0,1]$ is light.

Proposition 1.15. The space $C[0,1]$ is light.

Proof. Let $\left(T_{\alpha}\right)_{\alpha \in I}$ be a net in $\operatorname{Isom}(C[0,1])$ such that $T_{\alpha} \stackrel{\text { WOT }}{\longrightarrow}$ Id. By Banach-Stone Theorem, for every $\alpha \in I$ there exists a homeomorphism $\varphi_{\alpha}:[0,1] \rightarrow[0,1]$ and $h_{\alpha} \in C[0,1]$ with $\left|h_{\alpha}(x)\right|=1$, for every $x \in[0,1]$, such that

$$
T_{\alpha}(f)(x)=h_{\alpha}(x) f\left(\varphi_{\alpha}(x)\right)
$$

for every $f \in C[0,1]$ and $x \in[0,1]$. Since $T_{\alpha} \stackrel{\text { WOT }}{\longrightarrow}$ Id, taking $\Phi_{x} \in C[0,1]^{*}$ given by $\Phi_{x}(f)=f(x)$, it follows that $T_{\alpha}(f)(x) \rightarrow f(x)$ for every $x \in[0,1]$. In particular, taking $f=\mathrm{Id}$, we see that

$$
T_{\alpha}(\mathrm{Id})(x)=h_{\alpha}(x) \varphi_{\alpha}(x) \rightarrow x,
$$

for every $x \in[0,1]$. Without loss of generality, we may assume that $\varphi_{\alpha}$ is an increasing homeomorphism and $h_{\alpha} \equiv 1$, for every $\alpha$. We claim that for every $\delta>0$ there exists $\alpha_{\delta} \in I$ such that

$$
\alpha \succcurlyeq \alpha_{\delta} \Longrightarrow\left|\varphi_{\alpha}(x)-x\right|<\delta
$$

for every $x \in[0,1]$. Indeed, there exists $n \in \mathbb{N}, n \geqslant 1$ such that $\frac{1}{2^{n-1}}<\delta$ and there exists $\alpha_{\delta} \in I$ such that $\left|\varphi_{\alpha}\left(\frac{k}{2^{n}}\right)-\frac{k}{2^{n}}\right|<\frac{1}{2^{n+1}}$, for $k=0,1, \ldots, 2^{n}-1$ and $\alpha \succcurlyeq \alpha_{\delta}$. Since $\varphi_{\alpha}$ is an increasing function and $\frac{k}{2^{n}} \leqslant x \leqslant \frac{k+1}{2^{n}}$ for some $k$, it follows that

$$
\begin{aligned}
\left|\varphi_{\alpha}(x)-x\right| & \leqslant\left|\varphi_{\alpha}\left(\frac{k+1}{2^{n}}\right)-\frac{k}{2^{n}}\right| \\
& \leqslant\left|\varphi_{\alpha}\left(\frac{k}{2^{n}}\right)-\frac{k}{2^{n}}\right|+\left|\varphi_{\alpha}\left(\frac{k+1}{2^{n}}\right)-\frac{k+1}{2^{n}}\right|+\frac{1}{2^{n}} \\
& \leqslant \frac{1}{2^{n-1}} \\
& <\delta .
\end{aligned}
$$

Finally, by uniform continuity of $f \in C[0,1]$ we have

$$
\left\|T_{\alpha}(f)-f\right\|_{\infty}=\max _{0 \leqslant x \leqslant 1}\left|f\left(\varphi_{\alpha}(x)\right)-f(x)\right| \rightarrow 0
$$

i. e., $T_{\alpha} \stackrel{\text { SOT }}{\longrightarrow} \mathrm{Id}$.

It is interesting to notice that actually $C([0,1] \times F)$ is light for every finite set $F$, while $C\left([-1,1]^{2}\right)$ is not light.

Proposition 1.16. If $F$ is a finite set, then $C([0,1] \times F)$ is light. 
Proof. Let $F=\left\{y_{1}, \ldots, y_{n}\right\}$ be a finite set equipped with the discrete topology and let $\left(T_{\alpha}\right)_{\alpha \in I}$ be a net in $\operatorname{Isom}\left(C([0,1] \times F)\right.$ such that $T_{\alpha} \stackrel{\text { wOT }}{\longrightarrow}$ Id. By the Banach-Stone Theorem, for each $\alpha \in I$ there exists a homeomorphism $\varphi_{\alpha}:[0,1] \times F \rightarrow[0,1] \times F$ and $h_{\alpha} \in C([0,1] \times F)$ with $\left|h_{\alpha}\left(x, y_{i}\right)\right|=1$ for every $\left(x, y_{i}\right) \in[0,1] \times F$ such that

$$
T_{\alpha}(f)\left(x, y_{i}\right)=h_{\alpha}\left(x, y_{i}\right) f\left(\varphi_{\alpha}\left(x, y_{i}\right)\right)
$$

for every $f \in C([0,1] \times F)$ and every $\left(x, y_{i}\right) \in[0,1] \times F$. Since $T_{\alpha} \stackrel{\text { WOT }}{\longrightarrow}$ Id, we have $T_{\alpha}(f)\left(x, y_{i}\right) \rightarrow f\left(x, y_{i}\right)$ for every $\left(x, y_{i}\right) \in[0,1] \times F$. In particular, considering $g \in$ $C([0,1] \times F)$ defined by $g\left(x, y_{i}\right)=x+i$ we see that

$$
T_{\alpha}(g)\left(x, y_{i}\right)=h_{\alpha}\left(x, y_{i}\right) g\left(\varphi_{\alpha}\left(x, y_{i}\right)\right) \rightarrow x+i,
$$

for every $\left(x, y_{i}\right) \in[0,1] \times F$. Therefore, we may assume that for every $\alpha$ and $i$ we have $h_{\alpha} \equiv 1$ and

$$
\varphi_{\alpha}\left(x, y_{i}\right)=\left(\varphi_{\alpha, i}(x), y_{i}\right)
$$

where $\varphi_{\alpha, i}:[0,1] \rightarrow[0,1]$ is an increasing homeomorphism. We conclude that $T_{\alpha} \stackrel{\text { SOT }}{\longrightarrow}$ Id proceeding as in the proof of Proposition 1.15

A similar proof works also for the space $C_{0}(\mathbb{R})$. Recall that if $K$ is a locally compact Hausdorff space, then $C_{0}(K)$ denotes the space of functions on $K$ that vanish at infinity, i.e., the space

$$
C_{0}(K)=\{f: K \rightarrow \mathbb{R} ; \forall \varepsilon>0, \exists Q \subset K \text { compact } ; x \notin Q \Longrightarrow|f(x)|<\varepsilon\} .
$$

$C_{0}(K)$ is a Banach space with the norm $\|f\|=\sup _{x \in K}|f(x)|$.

The version of Banach-Stone Theorem for $C_{0}(K)$ spaces was given by Novinger:

Theorem 1.17 (Novinger). Let $K$ and $Q$ be locally compact Hausdorff spaces. If $T$ is a linear isometry from $C_{0}(Q)$ onto $C_{0}(K)$, then $Q$ and $K$ are homeomorphic. Furthermore

$$
T f(t)=h(t) f(\varphi(t))
$$

for all $t \in K$, where $h$ is continuous on $K$ such that $|h(t)|=1$ for all $t \in K$ and $\varphi$ is a homeomorphism of $K$ onto $Q$.

Proof. See [FJ03, Corollary 2.3.12]

Proposition 1.18. The space $C_{0}(\mathbb{R})$ is light.

Proof. Let $\left(T_{\alpha}\right)_{\alpha \in I}$ be a net in $\operatorname{Isom}\left(C_{0}(\mathbb{R})\right)$ such that $T_{\alpha} \stackrel{\text { WOT }}{\longrightarrow}$ Id. By Novinger's Theorem for each $\alpha \in I$ there exists a homeomorphism $\varphi_{\alpha}: \mathbb{R} \rightarrow \mathbb{R}$ and a continuous function $h_{\alpha}: \mathbb{R} \rightarrow \mathbb{R}$ with $\left|h_{\alpha}(x)\right|=1$ for every $x \in \mathbb{R}$ such that

$$
T_{\alpha}(f)(x)=h_{\alpha}(x) f\left(\varphi_{\alpha}(x)\right)
$$


for every $f \in C_{0}(\mathbb{R})$ and $x \in \mathbb{R}$. Let $f \in C_{0}(\mathbb{R})$ and let $\varepsilon>0$. There exists $K>0$ such that $|f(x)|<\frac{\varepsilon}{3}$ whenever $|x|>K$. Let $g \in C_{0}(\mathbb{R})$ be such that:

a) $g(x)=f(x)$, if $|x| \leqslant K$;

b) $g(x)=0$, if $|x|>K+1$ and

c) $\|f-g\|_{\infty}<\frac{\varepsilon}{3}$.

Proceeding as in the proof of Proposition 1.15, we see that $\left\|T_{\alpha}(g)-g\right\|_{\infty} \rightarrow 0$, i.e., there exists $\alpha_{0} \in I$ such that $\left\|T_{\alpha}(g)-g\right\|_{\infty}<\frac{\varepsilon}{3}$ for every $\alpha \succcurlyeq \alpha_{0}$. Therefore,

$$
\left\|T_{\alpha}(f)-f\right\|_{\infty} \leqslant\left\|T_{\alpha}(f)-T_{\alpha}(g)\right\|_{\infty}+\left\|T_{\alpha}(g)-g\right\|_{\infty}+\|f-g\|_{\infty}<\varepsilon,
$$

i.e., $T_{\alpha} \stackrel{\text { SOT }}{\longrightarrow} \mathrm{Id}$.

\subsection{SOT-compact groups}

We finish this chapter presenting a condition on groups $G \leqslant G L(X)$ that implies the lightness of $G$ :

Proposition 1.19. Let $X$ be a Banach space and let $G \leqslant G L(X)$. If $G$ is SOT-compact, then $G$ is light.

Proof. Let $\left(T_{\alpha}\right)_{\alpha \in I}$ be a net in $G$ such that $T_{\alpha} \stackrel{\text { WOT }}{\longrightarrow}$ Id and suppose, by contradiction, that $T_{\alpha} \stackrel{\text { SOT }}{\longrightarrow}$ Id. Then, there exists an SOT-open neighborhood $U$ of Id such that $T_{\alpha} \notin U$ for infinite indices $\alpha \in I$. Let $J=\left\{\alpha \in I ; T_{\alpha} \notin U\right\}$. Notice that $J$ is a directed set and hence $\left(T_{\alpha}\right)_{\alpha \in J}$ is a subnet of $\left(T_{\alpha}\right)_{\alpha \in I}$. Since $G \backslash U$ is SOT-compact, then $\left(T_{\alpha}\right)_{\alpha \in J}$ has an accumulation point $T \in G \backslash U$. Therefore, $T$ is also an accumulation point of $\left(T_{\alpha}\right)_{\alpha \in I}$ in WOT, which is a Hausdorff topology. Hence, $T=\mathrm{Id}$, which is a contradiction, since $T \notin U$ and $\operatorname{Id} \in U$.

\section{Questions and comments}

Bellenot proved in [Bel86] that any separable real Banach space $(X,\|\cdot\|)$ admits an equivalent renorming $\|\cdot\|$ such that $\operatorname{Isom}(X,\|\cdot\|)=\{ \pm \mathrm{Id}\}$. Jarosz generalized Bellenot's result in [Jar88, proving that any Banach space $(X,\|\cdot\|)$ (separable or not, real or complex) admits an equivalent renorming $\|\cdot\|$ such that

$$
\operatorname{Isom}(X,\|\cdot\|)=\{\lambda \operatorname{Id}: \lambda \in \mathbb{K},|\lambda|=1\},
$$

where $\mathbb{K}=\mathbb{R}$ in the real case and $\mathbb{K}=\mathbb{C}$ in the complex case. Obviously the group $\{\lambda \operatorname{Id}: \lambda \in \mathbb{K},|\lambda|=1\}$ is light. Therefore, even if $(X,\|\cdot\|)$ does not have the PCP, $X$ 
admits an equivalent renorming $\|\cdot\|$ such that $\operatorname{Isom}(X,\|\cdot\|)$ is light (and still fails the PCP).

Although Megrelishvili has defined the concept of light groups only for locally convex spaces, we can expand his definition to quasi-normed spaces, even if they are not locally convex. One could ask whether the isometry groups of non-convex quasi-normed spaces are light/non-light in general, but the spaces $\ell_{p}$ and $L_{p}[0,1], 0<p<1$ show that we do not have such general result. The space $L_{p}[0,1]$ is not light, since $\left(L_{p}[0,1]\right)^{*}=\{0\}$ for $0<p<1$ (see [KPR84, p. 18]). On the other hand, by Banach-Lamperti Theorem, each $T \in \operatorname{Isom}\left(\ell_{p}\right), 0<p<1$, has the form $T\left(x_{n}\right)=\left(\epsilon_{n} x_{\sigma(n)}\right)$, where $\varepsilon_{n} \in\{ \pm 1\}$ and $\sigma: \mathbb{N} \rightarrow \mathbb{N}$ is a bijection. Using the fact that the dual space of $\ell_{p}$ can be isometrically identified with $\ell_{\infty}$, where $\Phi=\left(y_{0}, y_{1}, \ldots\right) \in \ell_{\infty}$ acts in an element $x=\left(x_{0}, x_{1}, \ldots\right) \in \ell_{p}$ by the formula $\Phi(x)=\sum_{i=0}^{\infty} y_{i} x_{i}$ (see [KPR84, p. 21]) we can verify that $\operatorname{Isom}\left(\ell_{p}\right)$ is light.

Proposition 1.16 was generalized by Grivaux in [AFGR17], proving that if $K$ is an infinite compact connected space, then $C(K)$ is light if $K$ is homeomorphic to a finite union of segments of $\mathbb{R}$, and $C(K)$ is not light if $K$ contains an $n$-dimensional region for some $n \geqslant 2, C(K)$.

In the next chapters we provide more examples of light/non-light groups of isometries, but using different techniques. Namely, we will show that $c$ is not light, while $c_{0}$ is light, as well as the Schreier spaces of finite order.

We were able to classify the isometry groups of $\ell_{p}$, with $0<p \leqslant \infty$ and of $L_{p}[0,1]$, with $0<p<\infty$ in this chapter. However, it remains to verify whether $L_{\infty}[0,1]$ is light or not.

Question 1.20. Is $L_{\infty}[0,1]$ light?

Another interesting space which could be investigated in this context is the Gurarij space, whose isometry group possesses a very rich structure. For example, the isometry group of the Gurarij space is a universal Polish group, i.e, every Polish group embeds there homeomorphically.

Question 1.21. Is the isometry group of the Gurarij space light?

The Holmes space is another interesting space to be investigated (see [Hol92]). It is the Banach space spanned by the universal Urysohn metric space. Urysohn space is universal for the separable metric spaces and has the property that any isometry between finite subsets can be extended to an isometry for the whole space.

Question 1.22. Is the isometry group of the Holmes space light? 
The main difficulties to answer these questions are to get characterizations of the isometry groups of these spaces, as well as a nice characterization of their duals. 


\section{Isometries of Schreier spaces}

In Chapter 1 we classified several classical Banach spaces in terms of being light, i.e., sequence spaces or spaces of continuous functions which norms are defined by an explicit formula. The purpose of this chapter is to classify Banach spaces in which norms are defined by a family of finite sets of natural numbers which are compact, hereditary and spreading. These spaces are called combinatorial spaces. More specifically, we are interested in classifying the Schreier spaces in terms of being light.

\subsection{Schreier spaces}

Banach and Saks proved in [BS30] that every bounded sequence in $L^{p}$, with $p>1$, has a subsequence such that its arithmetic means converge in norm. This property is currently called Banach-Saks property. They also asked whether this property is also valid for the space of continuous functions. This question was answered negatively by Schreier in [Sch30], constructing a sequence of functions $\left(f_{i}\right)$ in $C[0,1]$ that converges weakly to 0 but has no subsequence whose arithmetic means converge in norm. In his construction, Schreier considered finite sets of natural numbers $F$ such that card $F \leqslant \min F$. The family

$$
\mathcal{S}_{1}=\left\{F \in[\mathbb{N}]^{<\infty}: \operatorname{card} F \leqslant \min F\right\} \cup\{\emptyset\}
$$

is now called Schreier family, and its elements are called Schreier sets.

Nishiura and Waterman showed in [NW63] that every space with the Banach-Saks property is reflexive. On the other hand, Baernstein [Bae72] gave an example of a reflexive space that does not have the Banach-Saks property. In [Bea79] Beauzamy used a variation of Baernstein's construction to define the space $X_{\mathcal{S}_{1}}$ as the completion of $c_{00}$ in respect to the norm

$$
\|x\|_{X_{\mathcal{S}_{1}}}=\sup _{F \in \mathcal{S}_{1}} \sum_{k \in F}\left|x_{k}\right|, \quad x=\left(x_{1}, \ldots, x_{k}, \ldots\right) \in c_{00} .
$$

This space was called Schreier space, in [BL84]. Beauzamy proved that the interpolation space $\left(\ell_{1}, X_{\mathcal{S}_{1}}\right)_{\theta, p}$, for $0<\theta<1$ and $1<p<\infty$ is a reflexive space that does not have the Banach-Saks property, providing simpler examples than the one obtained by Baernstein.

Alspach and Argyros defined in [AA92] the higher order Schreier spaces as follows. Let $\mathcal{A}_{n}$ denote the set of finite subsets of $\mathbb{N}$ with cardinality less than $n$. Letting $\mathcal{S}_{0}=\mathcal{A}_{1}$ and supposing that $\mathcal{S}_{\alpha}$ has been defined for some countable ordinal $\alpha$, we define

$$
\mathcal{S}_{\alpha+1}=\left\{\bigcup_{i=1}^{n} E_{i}: n \leqslant E_{1}<E_{2}<\cdots<E_{n} \text { and } E_{i} \in \mathcal{S}_{\alpha}\right\} \cup\{\emptyset\}
$$


where $n \leqslant E_{1}$ denotes $n \leqslant \min E_{1}$ and $E_{i}<E_{i+1}$ denotes $\max E_{i}<\min E_{i+1}$. If $\alpha$ is a limit ordinal then we fix $\alpha_{n} \nearrow \alpha$ and define $\mathcal{S}_{\alpha}=\{\emptyset\} \cup\{F \subset \mathbb{N}$ : for some $n \geqslant 1, F \in$ $\mathcal{S}_{\alpha_{n}}$ and $\left.n \leqslant F\right\}$. We may assume (see for example [Cau17]), that for each $n \in \mathbb{N}$ we have $\mathcal{S}_{\alpha_{n}} \subset \mathcal{S}_{\alpha_{n+1}}$. We say that $\mathcal{S}_{\alpha}$ is the Schreier family of order $\alpha$. The Schreier space of order $\alpha$, denoted by $X_{\mathcal{S}_{\alpha}}$, is defined as the completion of $c_{00}$ with respect to the norm

$$
\|x\|_{X_{\mathcal{S}_{\alpha}}}=\sup _{F \in \mathcal{S}_{\alpha}} \sum_{k \in F}\left|x_{k}\right|, \quad x=\left(x_{1}, \ldots, x_{k}, \ldots\right) \in c_{00}
$$

We can verify by transfinite induction that each family $\mathcal{S}_{\alpha}$ is:

1. hereditary $\left(F \in \mathcal{S}_{\alpha}\right.$ and $\left.G \subset F \Longrightarrow G \in \mathcal{S}_{\alpha}\right)$;

2. spreading $\left(\left\{l_{1}, l_{2}, \ldots, l_{n}\right\} \in \mathcal{S}_{\alpha}\right.$ and $\left.l_{i} \leqslant k_{i} \Longrightarrow\left\{k_{1}, k_{2}, \ldots, k_{n}\right\} \in \mathcal{S}_{\alpha}\right)$;

3. compact, seen as a subset of $\{0,1\}^{\mathbb{N}}$, with the natural identification of $\mathcal{P}(\mathbb{N})$ with $\{0,1\}^{\mathbb{N}}$.

A collection $\mathcal{F}$ of finite subsets of $\mathbb{N}$ satisfying these three properties is called a regular family in BDHQ18. Given a regular family $\mathcal{F}$, the combinatorial Banach space $X_{\mathcal{F}}$ is defined to be the completion of $c_{00}$ with respect to the norm

$$
\|x\|_{X_{\mathcal{F}}}=\sup _{F \in \mathcal{F}} \sum_{k \in F}\left|x_{k}\right|, \quad x=\left(x_{1}, x_{2}, \ldots\right) \in c_{00} .
$$

Combinatorial Banach spaces were first defined by Gowers in his blog [Gow09], for a system of natural numbers $\mathcal{A}$ that contains all the singletons and is hereditary. A more restrictive definition was given by Beanland, Duncan, Holt and Quigley in [BDHQ18, using regular families, with the objective of studying problems related to the cardinalities of the sets of extreme points of Banach spaces, especially Schreier spaces.

\subsection{Decompositions and spreads of Schreier sets}

In this section we will recall some useful properties of Schreier sets of finite order. We say that a set $F$ of a regular family $\mathcal{F}$ is maximal if there is no set $G \in \mathcal{F}$ that contains $F$ properly. We denote by $\mathcal{F}^{\text {max }}$ the collection of maximal sets of $\mathcal{F}$.

Proposition 2.1. Let $n \in \mathbb{N} \cup\{0\}$ and let $E \in \mathcal{S}_{n}^{\max }$. Then, for each $0 \leqslant m \leqslant n$ there exists a unique finite sequence $E_{1}<E_{2}<\cdots<E_{d}$ in $\mathcal{S}_{m}^{\max }$ such that $E=\bigcup_{i=1}^{d} E_{i}$. Moreover, $\left\{\min E_{i}\right\}_{i=1}^{d} \in \mathcal{S}_{n-m}^{\max }$.

Proof. The proof of this proposition is by induction over $n$. It is clear that it holds for $n=0$. Suppose that it holds for $0,1, \ldots n \in \mathbb{N} \cup\{0\}$ and let $E \in \mathcal{S}_{n+1}^{\max }$. By the 
definition of maximal Schreier sets, we can write $E$ in a unique way as $\bigcup_{i=1}^{d} E_{i}$, with $E_{1}<E_{2}<\cdots<E_{d} \in \mathcal{S}_{n}^{\max }$ and $\left\{\min E_{i}\right\}_{i=1}^{d} \in \mathcal{S}_{1}^{\max }$. Let $0 \leqslant m \leqslant n+1$. If $m=n+1$, the result is immediate. If $0 \leqslant m \leqslant n$, by induction hypothesis each $E_{i}$ can be written as $E_{i}=\bigcup_{j=1}^{a_{i}} E_{i, j}$, with $E_{i, 1}<E_{i, 2}<\cdots<E_{i, a_{i}} \in \mathcal{S}_{m}^{\max }$ and $\left\{\min E_{i, j}\right\}_{j=1}^{a_{i}} \in \mathcal{S}_{n-m}^{\max }$. Hence, $E$ can be written as the union of

$$
E_{1,1}<\cdots<E_{1, a_{1}}<E_{2,1}<\cdots<E_{2, a_{2}}<\cdots<E_{d, 1}<\cdots<E_{d, a_{d}} .
$$

Moreover, by the maximality of these sets, this decomposition is unique. Also, since $\left\{\min \left\{\min E_{i, j}\right\}_{j=1}^{a_{i}}\right\}_{i=1}^{d}=\left\{\min E_{i}\right\}_{i=1}^{d} \in \mathcal{S}_{1}^{\max }$, it follows that

$$
\left\{\min E_{i, j}: 1 \leqslant i \leqslant d, 1 \leqslant j \leqslant a_{i}\right\}=\bigcup_{i=1}^{d}\left\{\min E_{i, j}\right\}_{j=1}^{a_{i}} \in \mathcal{S}_{n+1-m}^{\max } .
$$

The converse of Proposition 2.1 also holds:

Proposition 2.2. Let $E=\bigcup_{i=1}^{d} E_{i}$, such that $E_{1}<E_{2}<\cdots<E_{d}$ is a finite sequence in $\mathcal{S}_{m}^{\max }$ and $\left\{\min E_{i}\right\}_{i=1}^{d} \in \mathcal{S}_{k}^{\max }$, with $m, k \geqslant 0$. Then, $E \in \mathcal{S}_{m+k}^{\max }$.

Proof. We will proceed the proof by induction over $k$. For $k=0$ it is immediate. Suppose that the result holds for some $k \in \mathbb{N} \cup\{0\}$ and for every $m \in \mathbb{N} \cup\{0\}$ and let $E_{1}<\cdots<$ $E_{d} \in \mathcal{S}_{m}^{\max }$ be such that $\left\{\min E_{i}\right\}_{i=1}^{d} \in \mathcal{S}_{k+1}^{\max }$. If $m=0$, the result is immediate. If $m \geqslant 1$, by Proposition 2.1 we can write $\left\{\min E_{i}\right\}_{i=1}^{d}=\bigcup_{j=1}^{r} F_{j}$, with $F_{1}<\cdots<F_{r} \in \mathcal{S}_{1}^{\max }$ and $\left\{\min F_{j}\right\}_{j=1}^{r} \in \mathcal{S}_{k}^{\max }$. For each $1 \leqslant j \leqslant r$, define

$$
G_{j}=\bigcup_{\min E_{i} \in F_{j}} E_{i} .
$$

Since $E_{i} \in \mathcal{S}_{m}^{\max }$ and $\left\{\min E_{i}: \min E_{i} \in F_{j}\right\}=F_{j} \in \mathcal{S}_{1}^{\max }$, it follows that $G_{j} \in \mathcal{S}_{m+1}^{\max }$. Moreover, since $E=\bigcup_{j=1}^{r} G_{j}$ and $\left\{\min G_{j}\right\}_{j=1}^{r}=\left\{\min F_{j}\right\}_{j=1}^{r} \in \mathcal{S}_{k}^{\max }$, by the induction hypothesis it follows that $E \in \mathcal{S}_{m+k+1}^{\max }$.

We say that $G=\left\{l_{1}, \ldots, l_{n}\right\} \subset \mathbb{N}$ is a spread of $F=\left\{k_{1}, \ldots, k_{m}\right\} \subset \mathbb{N}$ (written in increasing order) if $m=n$ and $k_{i} \leqslant l_{i}$ for each $1 \leqslant i \leqslant n$. Recall that the Schreier families are spreading, i.e., if $F \in \mathcal{S}_{n}$ and if $G$ is a spread of $F$, then $G \in \mathcal{S}_{n}$. In fact, we have a more general result:

Proposition 2.3. Let $n \in \mathbb{N}, E \in \mathcal{S}_{n}^{\max }$ and $F \subset \mathbb{N}$ such that $F$ is a spread of $E$, $\min E<\min F$ and card $E=\operatorname{card} F$. If $j>\min E$, then $\{j\} \cup F \in \mathcal{S}_{n}$.

Proof. By Proposition 2.1 we can write $E=\bigcup_{i=1}^{d} E_{i}$, with $E_{1}<\cdots<E_{d} \in \mathcal{S}_{n-1}^{\max }$ and $\left\{\min E_{i}\right\}_{i=1}^{d} \in \mathcal{S}_{1}^{\max }$. Hence, $\min E=\min E_{1}=d$. Since card $E=\operatorname{card} F$ and $F$ is a spread of $E$, we can write $F=\bigcup_{i=1}^{d} F_{i}$ such that for each $i$ the set $F_{i}$ is a spread of $E_{i}$. Therefore $\{j\}, F_{1}, \ldots, F_{d} \in \mathcal{S}_{n-1}$ and $\min \{j\} \cup F_{1} \cup \cdots \cup F_{d} \geqslant d+1$. Hence, $F \cup\{j\} \in \mathcal{S}_{n}$. 


\subsection{Isometry groups of Schreier spaces}

In Chapter 1 we classified several Banach spaces in terms of being light. However, all the isometry groups of those spaces were well known and could be found already in Banach's book Ban32. On the other hand, the isometry groups of Schreier spaces were not known until very recently. During the author's Doctoral Exchange Program (Programa de Doutorado Sanduíche no Exterior - PDSE-CAPES) in Washington and Lee University, we were trying to classify the Schreier spaces in terms of being light, but we needed the characterization of their isometry groups. Kevin Beanland and Hùng Việt $\mathrm{Chu}$, using properties of extreme points of $X_{\mathcal{S}_{\alpha}}$, managed to prove that for every $n \in \mathbb{N}$, the isometries of $X_{\mathcal{S}_{n}}$ consist only in change of signs of the coordinates.

For the sake of completeness, we will present their proof in Theorem 2.6. We will need the following properties of Schreier sets and extreme points of $X_{\mathcal{S}_{\alpha}}$. Recall that an extreme point of a convex set $C$ in a real vector space is a point in $C$ which does not lie in any open line segment joining two points of $C$, i.e., $x$ is an extreme point of $C$ if for any $y, z \in C$ such that

$$
x=\lambda y+(1-\lambda) z
$$

for some $0<\lambda<1$ we have $x=y=z$. The set of extreme points of $C$ is denoted by $\operatorname{ext}(C)$.

Theorem 2.4 (Beanland, Chu). Let $0<\alpha<\omega_{1}$ and let $x \in S_{X_{\mathcal{S}_{\alpha}}}$. Then $x \in \operatorname{ext}\left(B_{X_{\mathcal{S}_{\alpha}}}\right)$ if and only if the following conditions are satisfied:

a) $x \in c_{00}$;

b) there exists a non-maximal set $F \in \mathcal{S}_{\alpha}$ such that $\sum_{k \in F}\left|x_{k}\right|=1$;

c) for all $i \leqslant \max \operatorname{supp} x$ there exists $F \in \mathcal{S}_{\alpha}$ such that $i \in F$ and $\sum_{k \in F}\left|x_{k}\right|=1$, where supp $x$ denotes the support of $x$,

$$
\operatorname{supp} x=\left\{i \in \mathbb{N}: x_{i} \neq 0\right\} \text {. }
$$

Proof. See [ABC19, Theorem 2.6].

In the proof of Theorem 2.6 we will also need the following technical lemma, that is a consequence of results in Section 2.2 .

Lemma 2.5. Let $\left(k_{i}\right)_{i=1}^{\infty}$ be an increasing sequence in $\mathbb{N}$. For each $n \in \mathbb{N} \cup\{0\}$, let $\varphi_{n} \in \mathbb{N}$ such that $\left\{k_{i}\right\}_{i=1}^{\varphi_{n}} \in \mathcal{S}_{n}^{\max }$. 
1. If $F_{1}<F_{2}<\ldots$ is a sequence of finite subsets of $\mathbb{N}$ such that card $F_{i}=k_{i}$ and $k_{i}<F_{i}$ for every $i \geqslant 1$, then for every $j>k_{1}$ we have $\{j\} \cup \bigcup_{i=1}^{\varphi_{n-1}} F_{i} \in \mathcal{S}_{n}$;

2. If $G \in \mathcal{S}_{n}^{\max }$ can be written as $G=\bigcup_{i=1}^{m} G_{i}$, with $G_{1}<\cdots<G_{m} \in \mathcal{S}_{1}^{\max }$ and $\min G_{i} \leqslant k_{i}$ for every $1 \leqslant i \leqslant m$, then $m \leqslant \varphi_{n-1}$.

Proof.

1. For each $i \geqslant 1$ consider the sets $E_{i}=\left\{k_{i}\right\} \cup F_{i} \backslash\left\{\max F_{i}\right\}$. Notice that $E_{1}<E_{2}<$ $\cdots<E_{\varphi_{n-1}} \in \mathcal{S}_{1}^{\max }$ and that $\left\{\min E_{i}\right\}_{i=1}^{\varphi_{n-1}}=\left\{k_{i}\right\}_{i=1}^{\varphi_{n-1}} \in \mathcal{S}_{n-1}^{\max }$. By Proposition 2.2 it follows that $E=\bigcup_{i=1}^{\varphi_{n-1}} E_{i} \in \mathcal{S}_{n}^{\mathrm{MAX}}$. Moreover, since $F_{i}$ is a spread of $E_{i}$ and $\operatorname{card} E_{i}=\operatorname{card} F_{i}$ for each $i$, then $F=\bigcup_{i=1}^{\varphi_{n-1}} F_{i} \in \mathcal{S}_{n}$ is a spread of $E$ and card $E=\operatorname{card} F$. Since $\min E=k_{1}<\min F$, by Proposition 2.3 , it follows that for every $j>\min E=k_{1}$ we have $\{j\} \cup \bigcup_{i=1}^{\varphi_{n-1}} F_{i} \in \mathcal{S}_{n}$.

2. By Proposition 2.1, we have $\left\{\min G_{i}\right\}_{i=1}^{m} \in \mathcal{S}_{n-1}^{\max }$. Suppose by contradiction that $m>\varphi_{n-1}$. Since $\left\{k_{i}\right\}_{i=1}^{\varphi_{n-1}} \in \mathcal{S}_{n-1}^{\max }$ it follows that $\left\{k_{i}\right\}_{i=1}^{m} \notin \mathcal{S}_{n-1}$. However, since Schreier sets are spreading and $\min G_{i} \leqslant k_{i}$, we should have $\left\{\min G_{i}\right\}_{i=1}^{m} \notin \mathcal{S}_{n-1}$, which is a contradiction.

Using these properties, we can proceed to the main result:

Theorem 2.6. Let $n \in \mathbb{N}$ and $T \in \operatorname{Isom}\left(X_{\mathcal{S}_{n}}\right)$. Then, there exists a sequence $\left(\varepsilon_{i}\right)$ in $\{-1,1\}$ such that

$$
T(x)=\left(\varepsilon_{i} x_{i}\right)_{i=1}^{\infty},
$$

for every $x=\left(x_{i}\right)_{i=1}^{\infty} \in X_{\mathcal{S}_{n}}$.

Proof. Let $n \in \mathbb{N}$ and $T \in \operatorname{Isom}\left(X_{\mathcal{S}_{n}}\right)$. For each $i \in \mathbb{N}$, let

$$
T\left(e_{i}\right)=f_{i}=\left(f_{i, 1}, f_{i, 2}, f_{i, 3}, \ldots\right)
$$

and

$$
T^{-1}\left(e_{i}\right)=d_{i}=\left(d_{i, 1}, d_{i, 2}, d_{i, 3}, \ldots\right) .
$$

We will divide the proof of this theorem in eight steps:

Step 1: $f_{1}= \pm e_{1}$.

Suppose, by contradiction, that $f_{1, j} \neq 0$, for some $j \geqslant 2$. Let

$$
Y=\left\{x=\left(x_{1}, x_{2}, \ldots\right) \in X_{\mathcal{S}_{n}}: x_{1}=0\right\}
$$


Notice that, for every $x \in B_{Y}$ we have

$$
\left\|e_{1}+x\right\|=1=\left\|f_{1}+T x\right\| .
$$

Since $T(Y)$ is a subspace of codimension 1 of $X_{\mathcal{S}_{n}}$, there exists $k>j$ such that $e_{k} \in T(Y)$. On the other hand, since $\{j, k\} \in \mathcal{S}_{n}$, by (2.1) we have

$$
1=\left\|f_{1}+e_{k}\right\| \geqslant\left|f_{1, j}\right|+\left|1+f_{1, k}\right| \text {. }
$$

Moreover, there exist infinite $k>j$ such that $e_{k} \in T(Y)$ and $f_{1, k} \rightarrow 0$. Therefore,

$$
1=\left\|f_{1}+e_{k}\right\| \geqslant\left|f_{1, j}\right|+1
$$

which is a contradiction. Hence, $\operatorname{supp}\left(T\left(e_{1}\right)\right)=\{1\}$ and since $\left\|f_{1}\right\|=1$ we must have $f_{1}= \pm e_{1}$.

Step 2: $d_{k, 1}$ and $f_{k, 1}=0$, for every $k \geqslant 2$

Let $k \geqslant 2$. By Step 1 , there exists $\varepsilon_{1} \in\{-1,1\}$ such that $T\left(e_{1}\right)=f_{1}=\varepsilon_{1} e_{1}$. Thus, for every $k \geqslant 2$ we have

$$
\left\|e_{1}+e_{k}\right\|=\left\|\varepsilon_{1} e_{1}+f_{k}\right\|=1 \geqslant\left|\varepsilon_{1}+f_{k, 1}\right|
$$

and

$$
\left\|e_{1}-e_{k}\right\|=\left\|\varepsilon_{1} e_{1}-f_{k}\right\|=1 \geqslant\left|\varepsilon_{1}-f_{k, 1}\right| .
$$

These two inequalities imply that $f_{k, 1}=0$. In the same way we prove that $d_{k, 1}=0$.

Step 3: For every $i \in \mathbb{N}, d_{i}, f_{i} \in c_{00}$ and there exists a non-maximal set $F_{i} \in \mathcal{S}_{n}$ such that $\sum_{k \in F_{i}}\left|f_{i, k}\right|=1$.

It follows from the fact that isometries preserve extreme points, $e_{1}+e_{j} \in \operatorname{ext}\left(B_{X_{\mathcal{S}_{n}}}\right)$ for every $j \geqslant 2$ and from Theorem 2.4 .

$$
\text { Step 4: Let } m \in \mathbb{N} \text {. If } j>\max _{1 \leqslant i \leqslant m}\left\{\max \operatorname{supp} f_{i}\right\} \text {, then } \operatorname{supp} d_{j}>m \text {. }
$$

The proof of this step is by induction over $m$. For $m=1$, it follows from Step 2. Assume now that the conclusion holds for every $m^{\prime}<m$, for some $m \geqslant 2$. Fix $j>\max _{1 \leqslant i \leqslant m}\left\{\max \operatorname{supp} f_{i}\right\}$. By the induction hypothesis it follows that supp $d_{j}>m-1$, so it remains to prove that $d_{j, m}=0$. By Step 3, there exists a non-maximal set $F \in \mathcal{S}_{n}$ such that $\sum_{k \in F}\left|f_{m, k}\right|=1$. Thus $F \cup\{j\} \in \mathcal{S}_{n}$ and

$$
\left\|f_{m} \pm e_{j}\right\|=\sum_{k \in F \cup\{j\}}\left|f_{m, k}\right|+\delta_{j, k}=2=\left\|e_{m} \pm d_{j}\right\|
$$

where

$$
\delta_{j, k}=\left\{\begin{array}{l}
0, \text { if } j \neq k \\
1, \text { if } j=k
\end{array}\right.
$$


Suppose, by contradiction, that $m \in \operatorname{supp} d_{j}$. Since $\left\|e_{m} \pm d_{j}\right\|=2$, there exist sets $G$ and $H$ in $\mathcal{S}_{n}$ such that

$$
2-\left|d_{j, m}\right|<\sum_{i \in G}\left|\delta_{m, i}+d_{j, i}\right| \leqslant 2
$$

and

$$
2-\left|d_{j, m}\right|<\sum_{i \in H}\left|\delta_{m, i}-d_{j, i}\right| \leqslant 2 .
$$

Notice that if $m \notin G$, we would have

$$
1 \leqslant 2-\left|d_{j, m}\right|<\sum_{i \in G}\left|d_{j, i}\right| \leqslant 1
$$

which is a contradiction. For the same reason, we conclude that $m \in H$ and hence $m \in G \cap H$. If $d_{j, m}<0$ by 2.2 we would have

$$
2-\left|d_{j, m}\right|=2+d_{j, m}<1+d_{j, m}+\sum_{\substack{i \in G \\ i \neq m}}\left|d_{j, i}\right| \Longrightarrow 1<\sum_{\substack{i \in G \\ i \neq m}}\left|d_{j, i}\right|,
$$

which contradicts the fact that $\left\|d_{j}\right\|=1$. On the other hand, if $d_{j, m}>0$, by (2.3) we would have

$$
2-\left|d_{j, m}\right|=2-d_{j, m}<1-d_{j, m}+\sum_{\substack{i \in H \\ i \neq m}}\left|d_{j, i}\right| \Longrightarrow 1<\sum_{\substack{i \in H \\ i \neq m}}\left|d_{j, i}\right|,
$$

that also contradicts $\left\|d_{j}\right\|=1$. Therefore we must have $d_{j, m}=0$, i.e., $m \notin \operatorname{supp} d_{j}$, as desired.

Step 5: for every $r \in \mathbb{N}$, there exist $s, t \in \mathbb{N}$ such that

$$
r<\operatorname{supp} d_{s}<\operatorname{supp} d_{t}
$$

It follows from Step 4.

Step 6: for every $k \geqslant 2$, supp $f_{k} \leqslant k$.

By Step 5, we can find $F_{1} \subset \mathbb{N}$ with $k=k_{1}<\min F_{1}$, card $F_{1}=k_{1}, k_{1}<\operatorname{supp} \sum_{j \in F_{1}} d_{j}$ and such that $\left(d_{j}\right)_{j \in F_{1}}$ satisfies

$$
r, s \in F_{1}, r<s \Longrightarrow \operatorname{supp} d_{r}<\operatorname{supp} d_{s}
$$

Let

$$
k_{2}=\max \operatorname{supp} \sum_{j \in F_{1}} d_{j}=\max \operatorname{supp} d_{\max F_{1}}
$$

Continuing in this way, we obtain an increasing sequence $\left(k_{i}\right)_{i=1}^{\infty}$ in $\mathbb{N}$ and a sequence $\left(F_{i}\right)_{i=1}^{\infty}$ of finite subsets of $\mathbb{N}$ such that for every $i \geqslant 1$ :

a) $k_{i}<F_{i}$ 
b) $\operatorname{card} F_{i}=k_{i}$;

c) $k_{i}<\operatorname{supp} \sum_{j \in F_{i}} d_{j}$;

d) If $r, s \in F_{i}$ with $r<s$, then $\operatorname{supp} d_{r}<\operatorname{supp} d_{s}$.

For each $n \in \mathbb{N} \cup\{0\}$, let $\varphi_{n} \in \mathbb{N}$ such that $\left\{k_{i}\right\}_{i=1}^{\varphi_{n}} \in \mathcal{S}_{n}^{\max }$.

Claim: For every $G \in \mathcal{S}_{n}$ we have $k_{1} \notin G$ or $G \cap \operatorname{supp} d_{j}=\emptyset$ for some $j \in \bigcup_{i=1}^{\varphi_{n-1}} F_{i}$.

Indeed, suppose by contradiction that $k_{1} \in G$ and $G \cap \operatorname{supp} d_{j} \neq \emptyset$ for every $j \in \bigcup_{i=1}^{\varphi_{n-1}} F_{i}$. Without loss of generality, we may assume that $G$ is maximal in $\mathcal{S}_{n}$. By Proposition 2.1 we can write $G=\bigcup_{i=1}^{m} G_{i}$ with $G_{1}<G_{2}<\cdots<G_{m} \in \mathcal{S}_{1}^{\max }$ and $\left\{\min G_{i}\right\}_{i=1}^{m} \in \mathcal{S}_{n-1}^{\max }$. Since $k_{1} \in G$, it follows that $\min G_{1} \leqslant k_{1}$ and hence card $G_{1} \leqslant k_{1}$. The conditions $\operatorname{card} G_{1} \leqslant k_{1}=\operatorname{card} F_{1} ; k_{1}<F_{1} ; \operatorname{supp} d_{r}<\operatorname{supp} d_{s}$, if $r<s ; G \bigcap \operatorname{supp} d_{j} \neq \emptyset$; and $G_{1}<G_{2}<\cdots<G_{m}$, implie that

$$
G_{1} \bigcap \operatorname{supp} d_{\max F_{1}}=\emptyset .
$$

Therefore, we must have $G_{i} \cap \operatorname{supp} d_{\max F_{1}} \neq \emptyset$, for some $i>1$. Hence, $\min G_{i} \leqslant$ $\max \operatorname{supp} d_{\max F 1}$ which implies that $\min G_{2} \leqslant \max \operatorname{supp} d_{\max F 1}=k_{2}$.

Continuing in this manner we see that

$$
G_{i} \cap \operatorname{supp} d_{\max F_{i}}=\emptyset \quad \text { and } \quad \min G_{i} \leqslant k_{i}
$$

for each $1 \leqslant i \leqslant m$. By Lemma 2.5 item 2 , it follows that $m \leqslant \varphi_{n-1}$. However, $G_{m} \cap$ supp $d_{\max F_{m}}=\emptyset$ implies that $G \cap \operatorname{supp} d_{\max F_{m}}=\emptyset$ which contradicts our assumption and concludes the proof of the claim.

Since $\left\|d_{j}\right\|=1$ for every $j$, it follows from the claim that for every $G \in \mathcal{S}_{n}$ we have

$$
\sum_{r \in G}\left|\delta_{k, r}+\sum_{i=1}^{\varphi_{n-1}} \sum_{j \in F_{i}} d_{j, r}\right|<\sum_{i=1}^{\varphi_{n-1}} \operatorname{card} F_{i}
$$

Therefore,

$$
\left\|e_{k}+\sum_{i=1}^{\varphi_{n-1}} \sum_{j \in F_{i}} d_{j}\right\|=\sup _{G \in \mathcal{S}_{n}}\left\{\sum_{r \in G}\left|\delta_{k, r}+\sum_{i=1}^{\varphi_{n-1}} \sum_{j \in F_{i}} d_{j, r}\right|\right\} \leqslant \sum_{i=1}^{\varphi_{n-1}} \operatorname{card} F_{i} .
$$

Suppose by contradiction that $\max \operatorname{supp} f_{k}>k$. By Lemma 2.5 item 1, for every $j \in$ $\operatorname{supp} f_{k}$, with $j>k$, we have $\{j\} \cup \bigcup_{i=1}^{\varphi_{n-1}} F_{i} \in \mathcal{S}_{n}$. Then,

$$
\left\|e_{k}+\sum_{i=1}^{\varphi_{n-1}} \sum_{l \in F_{i}} d_{l}\right\|=\left\|f_{k}+\sum_{i=1}^{\varphi_{n-1}} \sum_{l \in F_{i}} e_{l}\right\| \geqslant\left|f_{k, j}\right|+\sum_{i=1}^{\varphi_{n-1}} \operatorname{card} F_{i}>\sum_{i=1}^{\varphi_{n-1}} \operatorname{card} F_{i},
$$

which contradicts 2.5. Hence, we must have max supp $f_{k} \leqslant k$. 
Step \%: $f_{2}= \pm e_{2}$.

It follows from Steps 2 and 6.

Step 8: $f_{k}= \pm e_{k}$, for every $k \geqslant 3$.

This final step is proved by induction. We proved in Steps 1 and 7 that $f_{1}= \pm e_{1}$ and $f_{2}= \pm e_{2}$. Let $k \geqslant 3$ and suppose that $f_{k^{\prime}}= \pm e_{k^{\prime}}$ for every $k^{\prime}<k$. If $j>k$, then $f_{k, j}=0$ by Step 6 . On the other hand, if $1 \leqslant j<k$, arguing as in Step 2 we conclude that $f_{k, j}=0$. Since $\left\|f_{k}\right\|=1$ we must have $\left|f_{k, k}\right|=1$, as desired.

\subsection{Lightness of $X_{\mathcal{S}_{n}}$}

The characterization of $\operatorname{Isom}\left(X_{\mathcal{S}_{n}}\right)$ given by Theorem 2.6 finally allows us to classify the Schreier spaces of finite order in terms of being light.

Proposition 2.7. Let $n \in \mathbb{N}$. The isometry group $\operatorname{Isom}\left(X_{\mathcal{S}_{n}}\right)$ is light.

Proof. Let $\left(T_{\alpha}\right)_{\alpha \in I}$ be a net in $\operatorname{Isom}\left(X_{\mathcal{S}_{n}}\right)$ such that $T_{\alpha} \stackrel{\text { WOT }}{\longrightarrow}$ Id and suppose, by contradiction, that $T_{\alpha} \stackrel{\text { SOT }}{\rightarrow}$ Id. Then, there exist $x=\left(x_{1}, x_{2}, \ldots\right) \in X_{\mathcal{S}_{n}}, \delta>0$ and indices $\alpha_{1}, \alpha_{2}, \cdots \in I$ such that $\left\|T_{\alpha_{\ell}} x-x\right\|>\delta$, for every $\ell \in \mathbb{N}$. By Theorem 2.6, for each $\ell \in \mathbb{N}$ there exists a sequence $\left(\varepsilon_{1}^{\alpha_{\ell}}, \varepsilon_{2}^{\alpha_{\ell}}, \ldots\right)$ in $\{-1,1\}$ such that $T_{\alpha_{\ell}} e_{i}=\varepsilon_{i}^{\alpha_{\ell}} e_{i}$ for each $i \in \mathbb{N}$. Since $T_{\alpha} \stackrel{\text { WOT }}{\longrightarrow} \mathrm{Id}$, for every $m \in \mathbb{N}, e_{m}^{*}\left(T_{\alpha_{\ell}} x\right) \stackrel{\ell \rightarrow \infty}{\longrightarrow} x_{m}$. Hence, for every $m \in \mathbb{N}$, there exists $N \in \mathbb{N}$ such that $l \geqslant N$ implies $\left(T_{\alpha_{\ell}} x\right)_{k}=x_{k}$, for every $1 \leqslant k \leqslant m$. On the other hand, since $\left\|T_{\alpha_{\ell}} x-x\right\|>\delta$, for every $m \in \mathbb{N}$ there exists $F_{m} \in \mathcal{S}_{n}$ with $\operatorname{supp}\left(F_{m}\right)>m$ such that

$$
\sum_{k \in F_{m}}\left|\left(T_{\alpha_{\ell}} x\right)_{k}-x_{k}\right|=\sum_{k \in F_{m}} 2\left|x_{k}\right|>\frac{\delta}{2} .
$$

Hence, $x$ cannot be approximated by elements of $c_{00}$ with respect to the norm of $X_{\mathcal{S}_{n}}$, which is a contradiction.

\section{Questions and comments}

It is worth mentioning that by Remark 1.4 the Schreier spaces do not have PCP, since they have an isometric copy of $c_{0}$ (see [CS89, Proposition 0.13]). Hence, we cannot apply Theorem 1.5 to conclude that $\operatorname{Isom}\left(X_{\mathcal{S}_{n}}\right)$ is light.

Although Theorem 2.6 was proved only for Schreier spaces of finite order, we believe that it is valid for every $X_{\mathcal{S}_{\alpha}}$, for $\alpha<\omega_{1}$. Almost every step in the proof can be reproduced in the countable case, except for Step 6, in which Lemma 2.5 is used. For this reason, we ask:

Question 2.8. Is Lemma 2.5 valid for $X_{\mathcal{S}_{\alpha}}, \alpha<\omega_{1}$ ? What are the correspondent versions of results of Section 2.2 for infinite countable ordinals? 
Another natural question is whether Theorem 2.7 is true for every combinatorial space.

Question 2.9. Does there exist a combinatorial space that is not light?

It is worth mentioning that Tsirelson's space is light, since it is reflexive. However, Tsirelson's space is not a combinatorial space, according to the definition that we adopted here. 


\title{
3 Convexity of norms and light groups
}

The purpose of this chapter is to connect the concept of light group of isomorphisms with the existence of smooth renormings. According to Deville, Godefroy and Zizler [DGZ93],

\begin{abstract}
Questions concerning the supply of smooth functions on Banach spaces are of crucial importance in differential calculus. Smooth functions are usually obtained from smooth norms and smooth norms are in turn often constructed from dual rotund norms. The existence of equivalent smooth or rotund norms or nontrivial smooth functions on a particular Banach space depends on its structure and has in turn a profound impact on its geometry.
\end{abstract}

We will show in Theorem 3.3 that if $G \leqslant G L(X)$ and $X$ admits a $G$-invariant LUR renorming in $X$ (or just LUR on a dense subset of $S_{X}$ ), then $G$ is light. Corollary 3.11 shows that the converse does not hold.

\subsection{Convexity of norms in vector spaces}

Let $(X,\|\cdot\|)$ be a Banach space. We say that a norm $\|\cdot\|$ in $X$ is rotund or strictly convex if for every $x, y \in X$ such that

$$
\|x\|=\|y\|=1 \quad \text { and } \quad\|x+y\|=2
$$

we have $x=y$. The following conditions are equivalent(see [DGZ93, Chapter 2, Proposition 1.3]):

1. The norm $\|\cdot\|$ is strictly convex;

2. If $x$ and $y \in X$ are such that $2\|x\|^{2}+2\|y\|^{2}-\|x+y\|^{2}=0$, then $x=y$;

3. If $x$ and $y \in X$ are such that $\|x+y\|=\|x\|+\|y\|$ and $x, y \neq 0$, then $x=\lambda y$ for some $\lambda>0$.

Geometrically, $\|\cdot\|$ is strictly convex if, and only if, the unit sphere $S_{X}$ does not contain any non-degenerated line segment.

The next definition is stronger than the previous one. We say that the norm $\|\cdot\|$ in $X$ is locally uniformly convex (LUC) or locally uniformly rotund $(L U R)$ in $x_{0} \in X$ if for every sequence $\left\{x_{n}\right\} \subset X$ such that

$$
\lim \left\|x_{n}\right\|=\left\|x_{0}\right\| \quad \text { and } \quad \lim \left\|x_{0}+x_{n}\right\|=2\left\|x_{0}\right\|
$$


we have $\lim \left\|x_{n}-x_{0}\right\|=0$. If $\|\cdot\|$ is LUR in every $x_{0} \in X$, we simply say that the norm is LUR in $X$.

If $x_{0} \in S_{X}$, we have the following equivalent definitions (see [DGZ93, Chapter 2, Proposition 1.2].):

1. The norm $\|\cdot\|$ is LUR in $x_{0}$;

2. If $\left(x_{n}\right)$ is a sequence in $S_{X}$ and $\lim \left\|x_{n}+x_{0}\right\|=2$, then $\lim \left\|x_{n}-x_{0}\right\|=0$;

3. If $\left(x_{n}\right)$ is a sequence in $X$ such that $\lim 2\left(\left\|x_{0}\right\|^{2}+\left\|x_{n}\right\|^{2}\right)-\left\|x_{0}+x_{n}\right\|^{2}=0$, then $\lim \left\|x_{0}-x_{n}\right\|=0$.

Geometrically, the norm $\|\cdot\|$ is LUR in $x_{0} \in S_{X}$ if taking a variable chord in $S_{X}$ with a fixed endpoint in $x_{0}$, if the midpoint of the chord gets close to the sphere then the length of the chord goes to zero.

An example of Banach space with LUR is $\ell_{2}$, since for every $x, y \in \ell_{2}$ we have the parallelogram identity

$$
2\left(\|x\|^{2}+\|y\|^{2}\right)=\|x+y\|^{2}+\|x-y\|^{2} .
$$

On the other hand, $\ell_{1}$ is not even strictly convex.

The convexity of a norm is deeply connected with the differentiability of the norm: if the dual norm $\|\cdot\|^{*}$ is strictly convex in $X^{*}$, then $\|\cdot\|$ is Gateaux differentiable in $X$; moreover, if $\|\cdot\|$ is LUR in $X^{*}$, then $\|\cdot\|$ is Fréchet differentiable in $X$ (Propositions 1.5 and 1.6 in [DGZ93]). It also has connections with the coincidence of weak and norm topologies on $S_{X}$.

A norm $\|\cdot\|$ on a Banach space $X$ is said to have the Kadec-Klee property KKP if the relative norm and weak topologies on the unit ball $B_{X}$ coincide in the unit sphere $S_{X}$. If $\|\cdot\|$ is an LUR norm on $X$, then $(X,\|\cdot\|)$ has the KKP (see [DGZ93], Proposition $1.4)$.

\section{2 $G$-invariant renormings}

A common problem in geometry of Banach spaces consist in investigating conditions in which a norm can be replaced by an equivalent one, but with better geometric properties. Recall that two norms $\|\cdot\|$ and $\|\cdot\|$ in a vector space $X$ are equivalent if there exist constants $C_{1}$ and $C_{2}>0$ such that

$$
C_{1}\|x\| \leqslant\|x\| \leqslant C_{2}\|x\|
$$


for every $x \in X$. In particular, they induce the same topology on $X$. We say that $\|\cdot\|$ is a renorming of $(X,\|\cdot\|)$ if $\|\cdot\|$ and $\|\cdot\|$ are equivalent.

A fundamental result in renorming theory was proved by Kadec in [Kad59]:

Theorem 3.1 (Kadec). Every separable Banach space admits an LUR renorming.

The proof of Theorem 3.1 consists of the following steps:

- If $Y$ admits an LUR dual norm and $T \in B(Y, X)$ is $w^{*}-w$ continuous with dense image in $X$, then $X$ admits an LUR renorming;

- If $X$ is separable, there exists $T \in B\left(X^{*}, \ell_{2}\right) w^{*}-w$ continuous such that $T^{*}: \ell_{2} \rightarrow X$ is $w^{*}-w$ continuous and $T^{*}\left(\ell_{2}\right)$ is dense in $X$;

- The usual norm of $\ell_{2}$ is LUR.

For the full proof, see DGZ93, Chapter 2, Theorem 2.6].

In Lan93] Lancien proves that if, in addition of being separable the space has the $\mathrm{RNP}$, then this renorming could be taken preserving the original isometries of the space.

We say that the norm $\|\cdot\|$ on $X$ is $G$-invariant, where $G \leqslant G L(X)$, if

$$
\|T x\|=\|x\|,
$$

for every $T \in G$ and every $x \in X$. In other words, $\|\cdot\|$ is $G$-invariant if $G$ is a subgroup of $\operatorname{Isom}(X,\|\cdot\|)$. If $\|\cdot\|$ is an equivalent norm of a Banach space $(X,\|\cdot\|)$ such that

$$
\operatorname{Isom}(X,\|\cdot\|) \subseteq \operatorname{Isom}(X,\|\cdot\|)
$$

we say that $\|\cdot\|$ is an isometry invariant renorming of $X$.

Lancien proves the following:

Theorem 3.2 (Lancien). If $X$ is a separable Banach space with RNP, then $X$ admits an isometry invariant LUR renorming.

The proof of this theorem is based on the concept of dentability index $\delta(X)$ of a Banach space. If $C$ is a closed and bounded subset of a Banach space $X$, consider the sections

$$
S(y, a)=\{x \in C ; y(x)>a\},
$$

where $y \in X^{*}$ and $a \in \mathbb{R}$. For $\varepsilon>0$, define

$$
C_{\varepsilon}^{\prime}=\{x \in C \text {; every section of } C \text { containing } x \text { has diameter }>\varepsilon\}
$$


For $\alpha$ ordinal, we define inductively $F_{\varepsilon}^{\alpha}$ in the following way:

$$
\begin{gathered}
F_{\varepsilon}^{0}=F=B_{X} \\
F_{\varepsilon}^{\alpha+1}=\left(F_{\varepsilon}^{\alpha}\right)_{\varepsilon}^{\prime} \\
F_{\varepsilon}^{\alpha}=\bigcap_{\beta<\alpha} F_{\varepsilon}^{\beta} \text {, if } \alpha \text { is a limit ordinal. }
\end{gathered}
$$

Set

$$
\delta(X, \varepsilon)=\left\{\begin{array}{l}
\left.\inf \alpha<\omega_{1} ; F_{\varepsilon}^{\alpha}=\varnothing\right\}, \text { if there exists; } \\
\omega_{1}, \text { otherwise. }
\end{array}\right.
$$

Finally, the dentability index of $X$ is defined as

$$
\delta(X)=\sup _{\varepsilon>0} \delta(X, \varepsilon)
$$

Lancien proves that if $X$ is separable and has the RNP, then $\delta(X)<\omega_{1}$. Moreover, if $\delta(X)<\omega_{1}$ then $X$ admits an LUR renorming. In addition, this renorming preserves isometries.

In [FR11] Ferenczi and Rosendal proved that $L_{1}[0,1]$ does not admit any isometry invariant LUR renorming, using the fact that the norm of $L_{1}[0,1]$ is almost transitive, which implies that any isometry invariant renorming must be a multiple of the original norm. On the other hand, the norm of $L_{1}[0,1]$ is nowhere LUR. Hence, Lancien's result does not generalize to every separable Banach space.

We will give another example of a separable space that does not admit any isometry invariant LUR renorming in Proposition 3.10, namely, the space $C[0,1]$. The proof of this theorem is based on the fact that $\operatorname{Isom}(C[0,1])$ is light (Proposition 1.15) and in next theorem:

Theorem 3.3. If a Banach space $X$ admits a G-invariant renorming $\|\cdot\|$ which is LUR in a dense subset of $S_{X}$, then $G$ is light.

Proof. Let $\left(T_{\alpha}\right)_{\alpha \in I}$ be a net in $G$ such that $T_{\alpha} \stackrel{\text { WOT }}{\longrightarrow}$ Id and suppose, by contradiction, that $T_{\alpha} \stackrel{\text { SOT }}{\rightarrow}$ Id. Then, there exists $x \in S_{X}$ such that $T_{\alpha} x \not \rightarrow x$. Without loss of generality, we may assume that there exists $\delta>0$ such that $\left\|T_{\alpha} x-x\right\| \geqslant \delta>0$ for every $\alpha$ and $\|\cdot\|$ is LUR in $x$. By the LUR property of $\|\cdot\|$ in $x$, we may also assume that there exists $\varepsilon>0$ such that, for every $\alpha \in I$ we have

$$
\left\|T_{\alpha} x+x\right\| \leqslant 2-\varepsilon
$$

Let $\phi \in X^{*}$ such that $\|\phi\|=1$ and $\phi(x)=1$. By the WOT convergence of $T_{\alpha}$ to Id, we have

$$
\phi\left(T_{\alpha} x\right) \rightarrow 1
$$


On the other hand,

$$
\left|\phi\left(T_{\alpha} x\right)+1\right|=\max _{\substack{\psi \in X^{*} \\\|\psi\|=1}}\left|\psi\left(T_{\alpha} x+x\right)\right|=\left\|T_{\alpha} x+x\right\| \leqslant 2-\varepsilon,
$$

which contradicts 3.1 .

Remark 3.4. In fact, the proof of Theorem 3.3 gives us a formally stronger result: if $X$ admits a $G$-invariant renorming which is LUR on a dense subset of $S_{X}$ then $G$ is orbit-wise light. Megrelishvili defines in Meg03 a group $G \leqslant G L(X)$ as orbit-wise light (or orbit-wise Kadec) if for every $x \in X$ the orbit $\mathcal{O}(x)=\{T x ; T \in G\}$ is a set on which the weak and the strong topologies coincide. It is readily seen that if $G$ is orbit-wise light, then it is light, but whether the converse holds is still an open question.

An example of application of Theorem 3.3 is to prove that $c_{0}$ is light. Indeed, Day defined in Day55 the following renorming of $c_{0}$ :

$$
\|x\|_{D}=\sup \left\{\left(\sum_{k=1}^{n} \frac{x_{\sigma(k)}^{2}}{4^{k}}\right)^{\frac{1}{2}}\right\},
$$

where the supremum is taken over all permutations $\sigma: \mathbb{N} \rightarrow \mathbb{N}$. By Novinger's Theorem, every isometry $\operatorname{Isom}\left(c_{0}\right)$ can be written as

$$
T\left(x_{n}\right)=\left(\varepsilon_{n} x_{\sigma(n)}\right)
$$

where $\sigma: \mathbb{N} \rightarrow \mathbb{N}$ is a permutation and $\left(\varepsilon_{n}\right)$ is a sequence such that $\left|\varepsilon_{n}\right|=1$ for every $n$. Therefore, Day's renorming is isometry invariant. Moreover, in [Rai69] Rainwater proved that this norm is LUR. Hence,

Corollary 3.5. The space $\left(c_{0},\|\cdot\|_{\infty}\right)$ is light.

\subsection{Renormings in $\ell_{\infty}$}

Day's norm can be defined in $\ell_{\infty}(\Gamma)$ in a similar way that it was defined for $c_{0}$, where $\Gamma$ is an arbitrary set. It also can be shown that it is LUR in $c_{0}(\Gamma)$, for every $\Gamma$ (see [DGZ93, p. 69-71]).

Although this norm is LUR in $c_{0}$, it is interesting to note that the same does not occur in $\ell_{\infty}$. In fact, it is not even strictly convex in $c$. For example, it is not strictly convex in $y=(1,1, \ldots)$ since for every $x=\left(x_{0}, x_{1}, \ldots\right) \in c$ such that $\|x\|_{\infty}=1$ and $\left|x_{i}\right|=1$ for infinite indices $i$, we have

$$
\|x\|_{D}=\|y\|_{D}=\left\|\frac{x+y}{2}\right\|_{D} .
$$


Proposition 1.10 and Theorem 3.3 imply that $\ell_{\infty}$ does not admit any isometry invariant LUR renorming. In fact, a stronger result is already known: the space $\ell_{\infty}$ does not admit any renorming with Kadec-Klee Property (see [DGZ93, Theorem 7.10, Chapter II]).

Since $\ell_{\infty}$ does not admit any renorming with KKP, it also does not admit any LUR renorming. On the other hand, $\ell_{\infty}$ admits strictly convex renormings (see [Die75, p. $120])$.

Question 3.6. Does $\ell_{\infty}$ admit a strictly convex isometry invariant renorming?

Next proposition gives a negative answer to this question.

Proposition 3.7. The space $\ell_{\infty}$ does not admit any strictly convex isometry invariant renorming.

Proof. Consider the points $x=(1,1,0,1,0,1,0, \ldots)$ and $y=(-1,1,0,1,0,1,0, \ldots) \in \ell_{\infty}$. Then,

$$
z=\frac{x+y}{2}=(0,1,0,1,0,1, \ldots) .
$$

Every $T \in \operatorname{Isom}\left(\ell_{\infty}\right)$ can be written as

$$
T\left(\left(x_{n}\right)_{n \in \mathbb{N}}\right)=\left(\varepsilon_{n} x_{\sigma(n)}\right)_{n \in \mathbb{N}}
$$

where $\sigma: \mathbb{N} \rightarrow \mathbb{N}$ is a permutation and $\left(\varepsilon_{n}\right)_{n \in \mathbb{N}}$ is a sequence in $\mathbb{K}$ such that $\left|\varepsilon_{n}\right|=1$,for every $n \in \mathbb{N}$ (see [Ban32, p. 178]). Therefore, there exist $T$ and $S \in \operatorname{Isom}\left(\ell_{\infty}\right)$ such that $T x=y$ and $S x=z$. Hence, if $\|\cdot\|$ is an isometry invariant renorming of $\ell_{\infty}$

$$
\|x\|=\|T x\|=\|y\|=\|S x\|=\|z\|,
$$

which implies that $\|\cdot\|$ cannot be strictly convex.

\subsection{A light space without isometry invariant LUR renormings}

In view of Theorem 3.3 , we may ask:

Question 3.8. Does there exist a Banach space $X$ and a bounded light group $G \leqslant G L(X)$ such that $X$ does not admit any LUR $G$-invariant renorming?

By Theorem 3.2 if such space exists it cannot have the RNP. Therefore, we need to look at the examples that we found previously of light groups of spaces without the RNP. Although $c_{0}$ is light, the group $\operatorname{Isom}\left(c_{0}\right)$ is not an answer to Question 3.8, since Day's renorming is LUR and isometry invariant. Another possibility is $\operatorname{Isom}(C[0,1])$, by Proposition 1.15. Indeed, in the same way we proved for $\ell_{\infty}$, we will show that $C[0,1]$ 
does not admit isometry invariant strictly convex renormings. We will need the following Lemma:

Lemma 3.9. Let $f \in C[0,1]$ be such that $f$ is strictly monotone in some interval $[a, b] \subset$ $[0,1]$. Then, there exists $g \in C[0,1]$ such that

$$
\begin{aligned}
& \text { 1. }\|f\|_{\infty}=\|g\|_{\infty}=\left\|\frac{f+g}{2}\right\|_{\infty} ; \\
& \text { 2. }\|f-g\|_{\infty}>0 ; \\
& \text { 3. } g=f \circ \varphi \text { and } \frac{f+g}{2}=f \circ \psi \text {, where } \varphi, \psi:[0,1] \rightarrow[0,1] \text { are homeomorphisms. }
\end{aligned}
$$

Proof. Let $0 \leqslant a<b \leqslant 1$ such that $\left.f\right|_{[a, b]}$ is strictly monotone. Without loss of generality, suppose that $f$ is strictly increasing in $[a, b]$. Let $\xi:[a, b] \rightarrow[f(a), f(b)]$ be an increasing homeomorphism such that $\left.\xi \not \equiv f\right|_{[a, b]}$. Define $g \in C[0,1]$ and the homeomorphism $\varphi$ : $[0,1] \rightarrow[0,1]$ by

$$
g(x)=\left\{\begin{array}{ll}
\xi(x), & \text { if } x \in[a, b] ; \\
f(x), & \text { otherwise. }
\end{array} \quad \varphi(x)= \begin{cases}f^{-1}(\xi(x)), & \text { if } x \in[a, b] \\
x, & \text { otherwise }\end{cases}\right.
$$

Then, $g=f \circ \varphi,\|g\|_{\infty}=\|f\|_{\infty}=\left\|\frac{f+g}{2}\right\|_{\infty}$ and $\|f-g\|_{\infty}>0$. Moreover, $f \circ \psi=\frac{f+g}{2}$, where $\psi:[0,1] \rightarrow[0,1]$ is the homeomorphism defined by

$$
\psi(x)= \begin{cases}f^{-1}\left(\frac{\xi(x)+f(x)}{2}\right), & \text { if } x \in[a, b] \\ x, & \text { otherwise }\end{cases}
$$

Proposition 3.10. Let $\|\cdot\|$ be an isometry invariant renorming of $C[0,1]$. Then, there exists a dense subset of $C[0,1]$ where $\|\cdot\| \|$ is not strictly convex.

Proof. Let $f \in C[0,1]$ be an affine non-constant function and let $g, \varphi$ and $\psi$ be as in Lemma 3.9. Since $f \mapsto f \circ \varphi$ and $f \mapsto f \circ \psi$ define surjective linear isometries on $C[0,1]$, then

$$
\|g\|=\|f \circ \varphi\|=\|f\|=\|f \circ \psi\|=\left\|\frac{f+g}{2}\right\|
$$

Hence, $\|\cdot\|$ is not strictly convex in $f$. The result follows from the fact that the set of piecewise linear continuous functions is dense in $C[0,1]$.

Corollary 3.11. The space $C[0,1]$ does not admit isometry invariant strictly convex renormings. 
Corollary 3.11 gives us a positive answer to Question 3.8. On the other hand, Remark 3.4 suggests the following new question:

Question 3.12. Does there exist a Banach space $X$ and an orbit-wise light group $G \leqslant$ $G L(X)$ such that $X$ admits no $G$-invariant LUR renorming?

The next proposition shows that the isometry group of $C[0,1]$ also gives a positive answer to Question 3.12 .

Proposition 3.13. The group $\operatorname{Isom}(C[0,1])$ is orbit-wise light.

Proof. Let $f \in C[0,1]$ and let $\left(g_{\alpha}\right)_{\alpha \in I}$ be a net in the orbit $\mathcal{O}(f)$ of $f$ under the action of the group $\operatorname{Isom}(C[0,1])$ such that $g_{\alpha}$ converges weakly to $g \in \mathcal{O}(f)$. By Banach-Stone Theorem, there exist homeomorphisms $\varphi, \varphi_{\alpha} \in \operatorname{Hom}([0,1])$ and $\varepsilon, \varepsilon_{\alpha} \in\{-1,1\}$ such that $g=\varepsilon \cdot f \circ \varphi$ and $g_{\alpha}=\varepsilon_{\alpha} \cdot f \circ \varphi_{\alpha}$. Since $g_{\alpha}$ converges weakly to $g$ (hence, pointwise), without loss of generality we can assume that the $\varphi_{\alpha}$ are increasing homeomorphisms, $\varepsilon=\varepsilon_{\alpha}=1$ for every $\alpha \in I$ and $g=f$.

Suppose by contradiction that $f \circ \varphi_{\alpha}$ does not converge uniformly to $f$. Then we can assume that there exists $\varepsilon>0$ and for every $\alpha \in I$ there exists $x_{\alpha} \in[0,1]$ such that $\left|f\left(\varphi_{\alpha}\left(x_{\alpha}\right)\right)-f\left(x_{\alpha}\right)\right|>2 \varepsilon$. We also can assume that $x_{\alpha} \rightarrow x \in[0,1]$ and $x_{\alpha} \leqslant x$ for every $\alpha$. Then by the continuity of $f$ at the point $x$,

$$
\left|f\left(\varphi_{\alpha}\left(x_{\alpha}\right)\right)-f(x)\right|>\varepsilon .
$$

Let $\delta>0$ be such that $|x-y|<\delta \Longrightarrow|f(x)-f(y)|<\frac{\varepsilon}{8}$. Then $\varphi_{\alpha}\left(x_{\alpha}\right) \notin(x-\delta, x+\delta)$ for every $\alpha$ and $\varphi_{\alpha}\left(x_{\alpha}\right)<x-\delta$ for infinitely many indices $\alpha \in I$, or $\varphi_{\alpha}\left(x_{\alpha}\right)>x+\delta$ for infinitely many indices $\alpha \in I$. Without loss of generality, we may assume that

$$
\varphi_{\alpha}\left(x_{\alpha}\right)<x-\delta, \quad \text { for every } \alpha \in I \text {. }
$$

We also may assume that

$$
x-\delta<x_{\alpha} \leqslant x, \quad \text { for every } \alpha \in I
$$

(the cases $\varphi_{\alpha}\left(x_{\alpha}\right)>x+\delta$ and/or $x<x_{\alpha}<x+\delta$ for every $\alpha \in I$ are similar).

Let $\alpha_{1} \in I$ and let

$$
y_{1,1}=\varphi_{\alpha_{1}}\left(x_{\alpha_{1}}\right)
$$

We claim that for every $n \geqslant 2$, there exists a finite sequence in $[0,1]$,

$$
y_{n, 1}<y_{n, 2}<\cdots<y_{n, 2 n-1}<x-\delta
$$

such that 
a) $\left|f\left(y_{n, 2 k+1}\right)-f(x)\right|>\varepsilon-\frac{\varepsilon}{8}-\frac{\varepsilon}{2^{k+4}}\left(\sum_{j=0}^{n-k-2} \frac{1}{2^{j}}\right)>\frac{3 \varepsilon}{4} \quad$ for $k=0,1, \ldots, n-1$ and

b) $\left|f\left(y_{n, 2 k}\right)-f(x)\right|<\frac{\varepsilon}{8}+\frac{\varepsilon}{2^{k+3}}\left(\sum_{j=0}^{n-k-1} \frac{1}{2^{j}}\right)<\frac{\varepsilon}{4} \quad$ for $k=1,2, \ldots, n-1$.

Notice that the existence of such a sequence for every $n \geqslant 2$ contradicts the uniform continuity of $f$ on $[0,1]$. Hence it suffices to prove the claim in order to complete the proof of Proposition 3.13 .

We proceed to the proof of the claim by induction. Since $f \circ \varphi_{\alpha}$ converges pointwise to $f$ and $x_{\alpha} \rightarrow x$, we can take $\alpha_{2} \succcurlyeq \alpha_{1}$ such that $x_{\alpha_{1}}<x_{\alpha_{2}}<x,\left|f\left(\varphi_{\beta}\left(y_{1,1}\right)\right)-f\left(y_{1,1}\right)\right|<\frac{\varepsilon}{16}$ and $\left|f\left(\varphi_{\beta}\left(x_{\alpha_{1}}\right)\right)-f\left(x_{\alpha_{1}}\right)\right|<\frac{\varepsilon}{16}$ for every $\beta \succcurlyeq \alpha_{2}$. Let

$$
y_{2,1}=\varphi_{\alpha_{2}}\left(y_{1,1}\right), \quad y_{2,2}=\varphi_{\alpha_{2}}\left(x_{\alpha_{1}}\right) \quad \text { and } \quad y_{2,3}=\varphi_{\alpha_{2}}\left(x_{\alpha_{2}}\right) .
$$

Since $\varphi_{\alpha_{2}}$ is an increasing homeomorphism and $y_{1,1}<x-\delta<x_{\alpha_{1}}<x_{\alpha_{2}}$, we have $y_{2,1}<y_{2,2}<y_{2,3}$ and $y_{2,3}=\varphi_{\alpha_{2}}\left(x_{\alpha_{2}}\right)<x-\delta$. Moreover,

$$
\left|f\left(y_{2,1}\right)-f(x)\right|>\varepsilon-\frac{\varepsilon}{8}-\frac{\varepsilon}{16}, \quad\left|f\left(y_{2,2}\right)-f(x)\right|<\frac{\varepsilon}{8}+\frac{\varepsilon}{16} \text { and }\left|f\left(y_{2,3}\right)-f(x)\right|>\varepsilon-\frac{\varepsilon}{8},
$$

which proves the inequalities for $n=2$.

Suppose now that the inequalities hold for $n$. Let $\alpha_{n+1} \succcurlyeq \alpha_{n}$ such that $x_{\alpha_{n}}<$ $x_{\alpha_{n+1}}<x,\left|f\left(\varphi_{\beta}\left(y_{n, r}\right)\right)-f\left(y_{n, r}\right)\right|<\frac{\varepsilon}{2^{n+3}}$ and $\left|f\left(\varphi_{\beta}\left(x_{\alpha_{n}}\right)\right)-f\left(x_{\alpha_{n}}\right)\right|<\frac{\varepsilon}{2^{n+3}}$ for every $r=1,2, \ldots, 2 n-1$ and every $\beta \succcurlyeq \alpha_{2}$. Let

$$
\begin{gathered}
y_{n+1, r}=\varphi_{\alpha_{n+1}}\left(y_{n, r}\right) \quad \text { for } r=1, \ldots, 2 n-1, \\
y_{n+1,2 n}=\varphi_{\alpha_{n+1}}\left(x_{\alpha_{n}}\right) \quad \text { and } \quad y_{n+1,2 n+1}=\varphi_{\alpha_{n+1}}\left(x_{\alpha_{n+1}}\right) .
\end{gathered}
$$

It follows that

$$
\begin{aligned}
\left|f\left(y_{n+1,2 k+1}\right)-f(x)\right| & >\varepsilon-\frac{\varepsilon}{8}-\frac{\varepsilon}{2^{k+4}}\left(\sum_{j=0}^{n-k-1} \frac{1}{2^{j}}\right) \text { for } k=0,1, \ldots, n \text { and } \\
\left|f\left(y_{n+1,2 k}\right)-f(x)\right| & <\frac{\varepsilon}{8}+\frac{\varepsilon}{2^{k+3}}\left(\sum_{j=0}^{n-k} \frac{1}{2^{j}}\right) \text { for } k=1,2, \ldots, n .
\end{aligned}
$$

Since $\varphi_{\alpha_{n+1}}$ is an increasing homeomorphism and $y_{n, 1}<y_{n, 2}<\cdots<y_{n, 2 n-1}<x-\delta<$ $x_{\alpha_{n}}<x_{\alpha_{n+1}}$, we have $y_{n+1,1}<y_{n+1,2}<\cdots<y_{n+1,2 n+1}=\varphi_{\alpha_{n+1}}\left(x_{\alpha_{n+1}}\right)<x-\delta$, which proves the claim. 


\section{Questions and comments}

The original proof of Theorem 3.3 was obtained by Ferenczi, Grivaux and Rosendal. We modified the proof here to include the complex case.

Megrelishvili proved that if $X$ has the PCP, then every bounded group of isomorphisms $G \leqslant G L(X)$ is light. We presented here two other conditions that imply the lightness of a bounded group: SOT-compactness (Proposition 1.19) and the existence of $G$-invariant LUR renormings (Theorem 3.3).

Question 3.14. What other conditions over $X$ and/or $G$ imply that a bounded group $G \geqslant G L(X)$ is light?

We were not able to find yet an orbit-wise light group that is not light. For this reason, we ask:

Question 3.15. Is every light group orbit-wise light? 


\section{Distinguished points and light groups}

Bellenot proved in [Bel86] that any separable real Banach space $(X,\|\cdot\|)$ admits an equivalent renorming $\|\cdot\|$ such that $\operatorname{Isom}(X,\|\cdot\|)=\{ \pm \mathrm{Id}\}$. Jarosz generalized Bellenot's result in [Jar88, proving that any Banach space $(X,\|\cdot\|$ ) (separable or not, real or complex) admits an equivalent renorming $\|\cdot\|$ such that

$$
\operatorname{Isom}(X,\|\cdot\|)=\{\lambda \operatorname{Id}: \lambda \in \mathbb{K},|\lambda|=1\}
$$

where $\mathbb{K}=\mathbb{R}$ in the real case and $\mathbb{K}=\mathbb{C}$ in the complex case. Ferenczi and Galego investigated in [FG10] what other groups may be seen as the group of isometries of a Banach space under some renorming. Among other results, they prove that if $X$ is a separable Banach space and $G$ is a finite group of isomorphisms of $X$ with $-\operatorname{Id} \in G$, then $X$ admits an equivalent norm $\|\cdot\|$ such that

$$
G=\operatorname{Isom}(X,\|\cdot\|)
$$

They also prove that if $X$ is a separable Banach space with LUR norm $\|\cdot\|$ and if $G$ is an infinite countable bounded isometry group of $X$ such that - Id $\in G$ and such that $G$ admits a point $x \in X$ with $\inf _{g \neq \mathrm{Id}}\|g x-x\|>0$, then $G=\operatorname{Isom}(X,\|\cdot\|)$ for some equivalent norm $\|\cdot\|$ on $X$. A point $x$ satisfying the condition

$$
\inf _{g \neq I d}\|g x-x\|>0
$$

is called in [FR11] a distinguished point of $X$ for the group $G$.

Ferenczi and Rosendal generalized results of [FG10] in [FR11] to certain uncountable Polish groups and also defined the concept of distinguished family for $X$ in relation to $G$ as a finite subset $F=\left\{x_{1}, \ldots, x_{n}\right\}$ of $X$ such that

$$
\inf _{T \neq \text { Id }}\left\{\max _{1 \leqslant i \leqslant n}\left\|T x_{i}-x_{i}\right\|\right\}>0 \text {. }
$$

In this chapter we will investigate the relations between the concepts of distinguished families and light groups. In Theorem 4.4 we will prove that if $G \leqslant G L(X)$ is such that $G$ has a distinguished point for $X$, but $G$ does not act as an SOT-discrete group on $X^{*}$, then $G$ is not light. In particular, the group $\operatorname{Isom}(c)$ is not light, which implies that every separable infinite dimensional $C(K)$ admits a non-light renorming (Corollary 4.9).

It is clear that if $G$ is an isometry group with a distinguished point, $G$ is discrete in the strong operator topology. Ferenczi and Rosendal ask in [FR11 if the converse of 
this fact holds, i.e., if every SOT-discrete group has a distinguished point. In Proposition 4.11 we will see that the answer to this question is negative. We will give an example of an infinite countable group of isomorphisms $G$ of $c_{0}$ which is SOT-discrete but does not admit a distinguished point for $G$. In addition, this group is also not light.

\subsection{Distinguished family for dual actions}

If $G \leqslant G L(X)$, besides the action of $G$ on $X$ we also may consider the action induced by $G$ on $X^{*}$, identifying $G$ with $\psi(G) \leqslant G L\left(X^{*}\right)$, where $\psi: G \rightarrow G L\left(X^{*}\right)$ is given by

$$
\psi(T)\left(x^{*}\right)=x^{*} \circ T^{-1}
$$

We will show in Proposition 4.3 that the existence of a distinguished family of cardinality $n$ in $X$ for $G$ implies the existence of a distinguished family of cardinality $n$ in $X^{*}$ for $G$. We will need the following lemmas:

Lemma 4.1. Suppose that $G \leqslant G L(X)$ is light. If $G$ acts like an SOT-discrete group on $X$, then $G$ acts like an SOT-discrete group on $X^{*}$.

Proof. Let $\psi: G \rightarrow G L\left(X^{*}\right)$ be defined by

$$
\psi(T)\left(x^{*}\right)=x^{*} \circ T^{-1}
$$

for every $T \in G$ and $x^{*} \in X^{*}$. We want to show that $\psi(G)$ is an SOT-discrete subgroup of $G L\left(X^{*}\right)$. Since $G$ is light, $G$ is WOT-discrete. Hence, for every $T \in G$ there exist $x_{1}, \ldots, x_{m} \in S_{X}, x_{1}^{*}, \ldots, x_{n}^{*} \in S_{X^{*}}$ and $\varepsilon>0$ such that

$$
G \cap\left\{S^{-1} \in G L(X) ;\left|x_{i}^{*}\left(S^{-1}\left(x_{j}\right)\right)-x_{i}^{*}\left(T^{-1}\left(x_{j}\right)\right)\right|<\varepsilon, 1 \leqslant i \leqslant n, 1 \leqslant j \leqslant m\right\}=\left\{T^{-1}\right\} .
$$

Notice that

$$
A=\left\{\Phi \in G L\left(X^{*}\right) ;\left\|\Phi\left(x_{i}^{*}\right)-\psi(T)\left(x_{i}^{*}\right)\right\|<\varepsilon, \quad 1 \leqslant i \leqslant n\right\}
$$

is an SOT-open subset of $G L\left(X^{*}\right)$. Moreover, if $\psi(S) \in \psi(G) \cap A$ then

$$
\left\|\psi(S)\left(x_{i}^{*}\right)-\psi(T)\left(x_{i}^{*}\right)\right\|<\varepsilon, \text { for every } 1 \leqslant i \leqslant n .
$$

Therefore,

$$
\left|\psi(S)\left(x_{i}^{*}\right)\left(x_{j}\right)-\psi(T)\left(x_{i}^{*}\right)\left(x_{j}\right)\right|<\varepsilon, \text { for every } 1 \leqslant i \leqslant n \text { and } 1 \leqslant j \leqslant m .
$$

Thus, $\mid x_{i}^{*}\left(S^{-1}\right)\left(x_{j}\right)-x_{i}^{*}\left(T^{-1}\left(x_{j}\right) \mid<\varepsilon\right.$ and by 4.1 , we conclude that $S=T$ and

$$
\psi(G) \cap A=\{\psi(T)\} .
$$

Hence, $\psi(G)$ is SOT-discrete in $G L\left(X^{*}\right)$. 
Lemma 4.2. Let $X$ be a Banach space, $G$ a bounded subgroup of $G L(X)$ and $\left\{x_{1}, \ldots, x_{n}\right\}$ a distinguished family of $X$ for the action of $G$. Let $\|\cdot\|$ be a $G$-invariant renorming of $X$ which is LUR in $x_{1}, \ldots, x_{n}$. Then, for any $\phi_{i} \in S_{X^{*},\|\cdot\|^{*}}$ with $\phi_{i} x_{i}=1, i=1, \ldots, n$, the family $\left\{\phi_{1}, \ldots, \phi_{n}\right\}$ is distinguished for the dual action of $G$ in $X^{*}$.

Proof. Without loss of generality, suppose that $\left\|x_{i}\right\|=1$ for every $i$. Let

$$
\gamma=\inf _{g \neq \mathrm{Id}} \max _{i}\left\|g x_{i}-x_{i}\right\|>0
$$

If $g \neq \mathrm{Id}$, let $i$ be such that $\left\|g x_{i}-x_{i}\right\| \geqslant \gamma$. Since $\|\cdot\|$ is LUR in $x_{1}, \ldots, x_{n}$, there exists $\varepsilon>0$, which depends of $\gamma$ but not of $i$, such that

$$
\left\|g x_{i}+x_{i}\right\| \leqslant 2-\varepsilon
$$

Hence,

$$
\begin{aligned}
\left|\phi_{i} g x_{i}+\phi_{i} x_{i}\right| & \leqslant 2-\varepsilon \\
\left|\phi_{i} g x_{i}+1\right| & \leqslant 2-\varepsilon \\
\phi_{i}\left(g x_{i}\right) & \leqslant 1-\varepsilon \\
g^{*}\left(\phi_{i}\right)\left(x_{i}\right)-\phi_{i}\left(x_{i}\right) & \leqslant-\varepsilon \\
\varepsilon & \leqslant\left\|g^{*}\left(\phi_{i}\right)-\phi_{i}\right\| .
\end{aligned}
$$

Lemmas 4.1 and 4.2 and Theorem 3.3 imply the following:

Proposition 4.3. Let $X$ be a Banach space, $G$ a bounded SOT-discrete subgroup of $G L(X)$ and suppose that $X$ admits a $G$-invariant renorming LUR in a dense subset of $S_{X}$. If there exists a distinguished family of cardinality $n$ for the action of $G$ on $X$, then there exists a distinguished family of cardinality $n$ for the dual action of $G$ on $X^{*}$.

The next theorem gives us a criterion to determine that a group is not light. We will use this result in Proposition 4.5 to prove that $c$ is not light.

Theorem 4.4. Let $G \leqslant G L(X)$ be such that $G$ has a distinguished point for $X$, but $G$ does not act as an SOT-discrete group on $X^{*}$. Then, $G$ is not light.

Proof. Let $x_{0}$ be a distinguished point of $X$ the action of $G$. Since

$$
\inf _{\substack{T \in G \\ T \neq \mathrm{Id}}}\left\|T x_{0}-x_{0}\right\|>0
$$

we have $T_{n} \stackrel{\text { SOT }}{\longrightarrow}$ Id for every sequence $\left(T_{n}\right)$ in $G$ such that $T_{n} \neq \operatorname{Id}$ for every $n$. On the other hand, since $G$ does not act as an SOT-discrete group on $X^{*}$, there exists a sequence 
$T_{n}^{*}$ on the dual representation $\psi(G)$ of $G$ on $G L\left(X^{*}\right)$ such that $T_{n}^{*} \neq \operatorname{Id}$ for every $n$ and $T_{n}^{*} \stackrel{\text { SOT }}{\longrightarrow} \mathrm{Id}$ in $\psi(G)$. Therefore, for every $\varphi \in X^{*}$,

$$
\begin{aligned}
& T_{n}^{*} \varphi \stackrel{\|\cdot\|_{X *}^{*}}{\longrightarrow} \varphi \\
& T_{n}^{*} \varphi(x) \rightarrow \varphi(x) \text {, for every } x \in B_{X} \\
& \varphi T_{n}^{-1}(x) \rightarrow \varphi(x) \text {, for every } x \in B_{X} \\
& T_{n}^{-1} \stackrel{\text { WOT }}{\longrightarrow} \mathrm{Id} \text {. }
\end{aligned}
$$

Proposition 4.5. There exists a subgroup $G$ of $\operatorname{Isom}(c)$ that has a distinguished point, but whose dual action on $\ell_{1}$ is not SOT-discrete.

Proof. Define $G$ as the subgroup of isometries $T$ of $c$ of the form

$$
T\left(\left(x_{k}\right)_{k \in \mathbb{N}}\right)=\left(\varepsilon_{k} x_{k}\right)_{k \in \mathbb{N}}, \quad\left(x_{k}\right)_{k \in \mathbb{N}} \in c,
$$

where the sequence $\left(\varepsilon_{k}\right)_{k} \in\{-1,1\}^{\mathbb{N}}$ is eventually constant. Notice that $(1,1, \ldots)$ is a distinguished point for $G$, since

$$
\inf _{\substack{T \in G \\ T \neq \operatorname{Id}}}\|T(1,1, \ldots)-(1,1, \ldots)\|=2 .
$$

On the other hand, the dual space of $c$ identifies isomorphically with $\ell_{1}$, where $\varphi=$ $\left(y_{k}\right)_{k \in \mathbb{N}} \in \ell_{1}$ acts on an element $x=\left(x_{k}\right)_{k \in \mathbb{N}} \in c$ by the formula

$$
\varphi(x)=y_{1} \lim _{k \rightarrow \infty} x_{k}+\sum_{k=2}^{\infty} y_{k} x_{k-1} .
$$

For every $n \in \mathbb{N}$, define the operator $T_{n} \in G$ by setting, for every $\left(x_{k}\right)_{k \in \mathbb{N}} \in c$,

$$
T_{n}\left(x_{1}, x_{2}, \ldots, x_{n-1}, x_{n}, x_{n+1}, \ldots\right)=\left(x_{1}, x_{2}, \ldots, x_{n-1},-x_{n}, x_{n+1}, \ldots\right) .
$$

For every $x \in c$ we have

$$
\varphi\left(T_{n}(x)\right)=y_{1} \lim _{k \rightarrow \infty}\left(T_{n}(x)\right)_{k}+\sum_{k=2}^{\infty} y_{k}\left(T_{n}(x)\right)_{k-1}=\left(y_{1} \lim _{k \rightarrow \infty} x_{k}+\sum_{k=2}^{\infty} y_{k} x_{k-1}\right)-2 y_{n} x_{n}
$$

which tends to $\varphi(x)$ as $n$ tends to infinity. Thus, the inequality

$$
\left\|T_{n}^{*} \varphi-\varphi\right\|_{X^{*}} \leqslant\left|\left(T_{n}^{*} \varphi-\varphi\right)(x)\right|=2\left|y_{n} x_{n}\right| \leqslant 2\left|y_{n}\right|\|x\|,
$$

$x \in c, \varphi \in \ell_{1}$, implies that $T_{n}^{*}$ tends SOT to Id, so the dual action of $G$ on $\ell_{1}$ is not SOT-discrete.

Remark 4.6. Since the dual action of the group $G$ of Proposition 4.5 is not SOT-discrete, by Lemma 4.1 its action on $c$ is also not SOT-discrete.

By Theorem 4.4 we also have the classification of $\operatorname{Isom}(c)$ in terms of being light:

Corollary 4.7. The space $c$ is not light. 


\subsection{Non-light renormings of $C(K)$}

We proved in Corollary 4.7 that $\operatorname{Isom}(c)$ is not light. In fact, we can use this result to prove that every infinite dimensional separable space $C(K)$ admits a renorming $\|\cdot\|$ such that $\operatorname{Isom}(C(K),\|\cdot\|)$ is not light. We will need the following result:

Proposition 4.8. Suppose that $Y$ is a complemented subspace of $X$. If every bounded group of isomorphisms of $X$ is light, then every bounded group of isomorphisms of $Y$ is light.

Proof. Let $Z$ be a closed subspace of $X$ such that $X \simeq Y \oplus Z$. Let $G \leqslant G L(Y)$ be a bounded subgroup and for each $T \in G$ define $\widetilde{T} \in G L(X)$ by $\widetilde{T}(x)=(T(y), z)$, where $x=(y, z) \in X \simeq Y \oplus Z$. Notice that $\widetilde{G}=\{\widetilde{T} ; T \in G\}$ is a bounded subgroup of $G L(X)$ and hence, light.

Let $\left(T_{\alpha}\right)_{\alpha \in I}$ be a net in $G$ such that $T_{\alpha} \stackrel{\text { WOT }}{\longrightarrow} \operatorname{Id}_{Y}$. We claim that $\widetilde{T}_{\alpha} \stackrel{\text { WOT }}{\longrightarrow} \operatorname{Id}_{X}$. Indeed, for every $\Phi=(\xi, \psi) \in X^{*} \simeq Y^{*} \oplus Z^{*}$ and every $x=(y, z) \in X$ we have

$$
\left|\Phi\left(\widetilde{T}_{\alpha}(x)\right)-\Phi(x)\right|=\left|\xi\left(T_{\alpha}(y)\right)-\xi(y)\right| \rightarrow 0 .
$$

Since $\widetilde{G}$ is light, then $\widetilde{T}_{\alpha} \stackrel{\text { SOT }}{\longrightarrow} \operatorname{Id}_{X}$. Moreover, for every $y \in Y$ we have

$$
\left\|T_{\alpha}(y)-y\right\|_{Y}=\left\|\widetilde{T}_{\alpha}(y, 0)-(y, 0)\right\|_{X} \rightarrow 0
$$

i.e., $T_{\alpha} \stackrel{\text { SOT }}{\longrightarrow} \operatorname{Id}_{Y}$.

Since $c$ is isomorphic to $c_{0}$ (see, [FHH ${ }^{+} 11$, p. 271, Exercise 5.16]) and $c_{0}$ is isomorphic to a complemented subspace of $C(K)$ for every infinite, compact and metrizable $K$ (see $\left[\mathrm{FHH}^{+} 11\right.$, p. 273, Exercise 5.26]), and since $C(K)$ is separable if and only if $K$ is metrizable (see [FHH ${ }^{+} 11$, p. 128, Lemma 3.102]), it follows:

Corollary 4.9. Every infinite dimensional separable $C(K)$ admits a non-light renorming.

Proof. Let $Z$ be a closed subspace of $C(K)$ such that $C(K) \simeq \operatorname{Isom}(c) \oplus Z$. Let

$$
G=\left\{g \oplus \operatorname{Id}_{Z} ; g \in \operatorname{Isom}(c)\right\}
$$

By Propositions 4.5 and 4.8 $G$ is a bounded non-light group of isomorphisms of $C(K)$. Moreover, $C(K)$ admits a LUR renorming $\|\cdot\|$, since it is separable (Theorem 3.1). Also,

$$
\|x\|=\sup _{g \in G}\|g x\|
$$

is a $G$-invariant LUR renorming of $C(K)$. Since $G \leqslant \operatorname{Isom}(X,\|\cdot\|)$, it follows that $\operatorname{Isom}(X,\|\cdot\|)$ is also non-light. 


\subsection{Example of an SOT-discrete group without distinguished points}

If $x_{0}$ is a distinguished point of $X$ for $G \leqslant G L(X)$, there exists $\varepsilon>0$ such that

$$
\inf _{\substack{T \in G \\ T \neq \mathrm{Id}}}\left\|T x_{0}-x_{0}\right\|>\varepsilon
$$

Hence, $A=\left\{T \in G:\left\|T x_{0}-x_{0}\right\|<\varepsilon\right\}$ is an SOT-open subset of $G$ that contains only the identity and therefore $G$ is SOT-discrete.

On the other hand, since the sets

$$
V_{x_{1}, \ldots, x_{n}, T, \varepsilon}=\left\{S:\left\|S x_{i}-T x_{i}\right\|<\varepsilon, \forall 1 \leqslant i \leqslant n\right\}
$$

form a basis for SOT, if $G \leqslant G L(X)$ is SOT discrete there exist $\varepsilon>0$ and $x_{1}, \ldots, x_{n} \in X$ such that

$$
G \cap V_{x_{1}, \ldots, x_{n}, \mathrm{Id}, \varepsilon}=\{\mathrm{Id}\} .
$$

Thus, $\left\{x_{1}, \ldots, x_{n}\right\}$ is a distinguished family of $X$ for the action of $G$. In [FR11] Ferenczi and Rosendal ask if we also can get a distinguished point for $G$ in this condition.

Question 4.10. If $G$ is an isomorphism group of $X$ which is SOT-discrete, should $X$ have a distinguished point for $G$ ?

Next proposition gives a negative answer to this question.

Proposition 4.11. For every $r \geqslant 2$, there exists a bounded infinite SOT-discrete isomorphism group which admits a distinguished family of cardinality $r$, but does not admit any distinguished family of cardinality $r-1$.

Proof. Let $\left(\lambda_{k}\right)_{k}, \lambda_{k}=\left(\lambda_{k}^{(1)}, \ldots, \lambda_{k}^{(r)}\right)$, be a dense sequence in the unit sphere $S^{r-1}$ of $\mathbb{R}^{r}$ for the $\ell_{1}$ norm. Consider the vectors of the canonical basis of $c_{00}\left(e_{n}\right)_{n \geqslant 1}$ and for each $k>r$ define the functionals

$$
x_{k}^{*}=\lambda_{k}^{(1)} e_{1}+\lambda_{k}^{(2)} e_{2}+\cdots+\lambda_{k}^{(r)} e_{r}+e_{k},
$$

which seen as an element of $\ell_{1}$ has norm at most $r+1$. For every $k>r$ define the operators $R_{k}: c_{0} \rightarrow c_{0}$ by

$$
R_{k}(x)=-2 x_{k}^{*}(x) e_{k}
$$

Notice that

$$
R_{k}^{2}=-2 R_{k} \quad \text { and } \quad R_{n} R_{k}=0, \quad \text { if } k \neq n .
$$

Thus

$$
\left(\mathrm{Id}+R_{k}\right)^{2}=\mathrm{Id}
$$


and if $n \neq k$ we have

$$
\left(\mathrm{Id}+R_{k}\right)\left(\mathrm{Id}+R_{n}\right)=\mathrm{Id}+R_{k}+R_{n}=\left(\mathrm{Id}+R_{n}\right)\left(\mathrm{Id}+R_{k}\right) .
$$

Therefore, the operators $T_{n}=\mathrm{Id}+R_{n}$ generate a countable infinite abelian subgroup $G \leqslant G L\left(c_{0}\right)$. Moreover, any element of $G$ can be written as

$$
T=\mathrm{Id}+\sum_{k \in F} R_{k}
$$

for some finite subset $F \subset \mathbb{N} \cap[r,+\infty)$. Hence, for every $x=\left(x_{n}\right)_{n} \in B_{c_{0}}$ we have

$$
\begin{aligned}
\|T x\|=\left\|x-2 \sum_{k \in F} x_{k}^{*}(x) e_{k}\right\| & \leqslant \max \left\{\|x\|, \max _{k \in F}\left|x_{k}-2 x_{k}^{*}(x)\right|\right\} \\
& \leqslant \max \left\{1, \max _{k \in F}\left|-x_{k}-2 \sum_{j \geqslant r} \lambda_{k}^{(j)} x_{j}\right|\right\} \\
& \leqslant 3 .
\end{aligned}
$$

It follows that $\|T\| \leqslant 3$ for every $T \in G$, i.e., $G$ is bounded. We will now prove that no family $\left\{x_{1}, \ldots, x_{r-1}\right\}$ of $c_{0}$ is distinguished. Notice that for every $k>r$ and $1 \leqslant s \leqslant r-1$ we have

$$
\begin{aligned}
\left\|\left(\operatorname{Id}+R_{k}\right)\left(x_{s}\right)-x_{s}\right\| & =2|| x_{k}^{*}\left(x_{s}\right) e_{k} \| \\
& =2\left|\lambda_{k}^{(1)} x_{s}^{(1)}+\cdots+\lambda_{k}^{(r)} x_{s}^{(r)}+x_{s}^{(k)}\right| \\
& \leqslant 2\left|\lambda_{k}^{(1)} x_{s}^{(1)}+\cdots+\lambda_{k}^{(r)} x_{s}^{(r)}\right|+2\left|x_{s}^{(k)}\right| .
\end{aligned}
$$

Since $\operatorname{dim} \operatorname{span}\left\{x_{1}, \ldots, x_{r-1}\right\} \leqslant r-1$ there exists $y=\left(y^{(1)}, \ldots, y^{(r)}\right) \in S^{r-1}$ such that $y \perp x_{s}$ for every $1 \leqslant s \leqslant r-1$. By the density of $\left(\lambda_{k}\right)_{k}$ in $S^{r-1}$, it follows that

$$
\liminf _{k}\left\|\left(\operatorname{Id}+R_{k}\right) x_{s}-x_{s}\right\|=0
$$

for every $1 \leqslant s \leqslant r-1$ and hence the family $\left\{x_{1}, \ldots, x_{r-1}\right\}$ is not distinguished. On the other hand, the family

$$
\left\{e_{1}, \ldots, e_{r}\right\}
$$

is distinguished. Indeed, notice that for every $T=\mathrm{Id}+\sum_{k \in F} R_{k}, F \neq \emptyset$, and $1 \leqslant s \leqslant r$ we have

$$
\left\|T e_{s}-e_{s}\right\|=2 \max _{k \in F}\left|\lambda_{k}^{(s)}\right|
$$

Since

$$
\left|\lambda_{k}^{(1)}\right|+\cdots+\left|\lambda_{k}^{(r)}\right|=1
$$

there exists $i \in\{1, \ldots, r\}$ such that $\left|\lambda_{k}^{(i)}\right| \geqslant \frac{1}{r}$. It follows that

$$
\inf _{\substack{T \in G \\ T \neq \mathrm{Id}}}\left\{\max _{1 \leqslant s \leqslant r}\left\|T e_{s}-e_{s}\right\|\right\} \geqslant \frac{2}{r}>0
$$

and $\left\{e_{1}, \ldots, e_{r}\right\}$ is distinguished for $G$. In particular, $G$ is SOT-discrete. 
Taking $r=2$ in Proposition 4.11, we have a negative solution to Question 4.10. Moreover, we have:

Proposition 4.12. The groups of Proposition 4.11 are not light.

Proof. For every $x \in c_{0}$,

$$
R_{n}(x)=-2\left(\lambda_{n}^{(1)} x^{(1)}+\lambda_{n}^{(2)} x^{(2)}+\cdots+\lambda_{n}^{(r)} x^{(r)}+x^{(n)}\right) e_{n}
$$

converges weakly to 0 , i.e., $T_{n} \stackrel{\text { WOT }}{\longrightarrow}$ Id. On the other hand, taking

$$
x=e_{1}+e_{2}+\cdots+e_{r}
$$

we have

$$
\left\|T_{n}(x)-x\right\|=\left\|R_{n}(x)\right\|=2\left|\lambda_{n}^{(1)}+\lambda_{n}^{(2)}+\cdots+\lambda_{n}^{(r)}\right| .
$$

By the density of sequence $\left(\lambda_{n}\right)_{n}$ in $S^{r-1}$ we cannot have

$$
\lim _{n \rightarrow \infty}\left|\lambda_{n}^{(1)}+\lambda_{n}^{(2)}+\cdots+\lambda_{n}^{(r)}\right|=0 .
$$

Therefore, $R_{n}(x)$ does not converge strongly to 0 and $T_{n} \stackrel{\text { SOT }}{\nrightarrow}$ Id.

\subsection{SOT-discrete groups with dense orbit}

A famous and still unsolved problem in geometry of Banach spaces is Mazur's rotation problem. This problem is motivated by the observation that the Hilbert space $H=L_{2}[0,1]$ has the following property: for every pair $f, g \in S_{H}$ there exists $T \in \operatorname{Isom}(H)$ such that $T(f)=g$. Indeed, if $f= \pm g$, then \pm Id would be an isometry that maps $f$ in $g$. Otherwise, let $Y=\operatorname{span}\{f, g\}$. Define an isometry $T \in \operatorname{Isom}(Y)$ such that $T(f)=g$ and extend it linearly to $H=Y \oplus Y^{\perp}$ using the identity in $Y^{\perp}$. Denote by $\tilde{T}$ this extension. Then, for every $h=(u, v) \in Y \oplus Y^{\perp}$ we have

$$
\begin{aligned}
\|\tilde{T}(u, v)\|^{2} & =\langle\tilde{T}(u+v), \tilde{T}(u+v)\rangle \\
& =\langle T u+v, T u+v\rangle \\
& =\langle T u, T u\rangle+\langle v, v\rangle \\
& =\langle u, u\rangle+\langle v, v\rangle \\
& =\langle u+v, u+v\rangle \\
& =\|u+v\|^{2}
\end{aligned}
$$

i.e., $\tilde{T} \in \operatorname{Isom}(H)$. In [Ban32, p. 242] Banach attributes to Mazur the following question: Question 4.13 (Mazur's rotation problem). Let $X$ be a separable infinite dimensional space such that, for every $x, y \in S_{X}$ there exists $T \in \operatorname{Isom}(X)$ with $T x=y$. Is $X$ isometric to $H$ ? 
In modern terminology, Mazur asks if every separable infinite dimensional transitive Banach space is isometric to $L_{2}[0,1]$. A norm $\|\cdot\|$ in $X$ is called transitive if for every $x, y \in X$ there exists $T \in \operatorname{Isom}(X)$ such that $T x=y$. In other words, $\|\cdot\|$ is transitive if the orbit of a point $x_{0} \in S_{X}$ (or equivalently, of every point $x \in S_{X}$ ) by the group action of $\operatorname{Isom}(X)$

$$
\mathcal{O}\left(x_{0}\right)=\operatorname{Isom}(X) x_{0}=\left\{T x_{0} ; T \in \operatorname{Isom}(X)\right\}
$$

is equal to the set $S_{X}$. When $\mathcal{O}\left(x_{0}\right)$ is only a dense subset of $S_{X}$, we say that the norm $\|\cdot\|$ is almost transitive.

Mazur's rotation problem motivates many works about the existence of transitive or almost transitive renormings of Banach spaces (see, e.g., [FR13]).

Proposition 4.5 gives us an example of SOT-discrete isometry group that has a distinguished point $x_{0} \in S_{X}$. In particular, the orbit $\mathcal{O}\left(x_{0}\right)$ is not dense in $S_{X}$. A natural question that arises from this fact is the following:

Question 4.14. Does there exist a Banach space $X$ such that $\operatorname{Isom}(X)$ is SOT-discrete but whose orbit for some $x_{0} \in S_{X}$ is dense in $S_{X}$ ?

We will give a partial answer to this question in Proposition 4.16, if such space does exist, then its isometry group cannot be abelian. This an immediate consequence of the following lemma:

Lemma 4.15. Let $X$ be a Banach space such that $\operatorname{Isom}(X)$ acts almost-transitively on $S_{X}$. If there exists a distinguished family $\left\{x_{1}, \ldots, x_{n}\right\} \subset S_{X}, n \geqslant 2$ for $\operatorname{Isom}(X)$, then $\operatorname{Isom}(X)$ is not abelian.

Proof. Let $\varepsilon>0$ be such that

$$
\inf _{T \neq \mathrm{Id}}\left\{\max _{1 \leqslant i \leqslant n}\left\|T x_{i}-x_{i}\right\|\right\} \geqslant \varepsilon .
$$

Since

$$
\mathcal{O}\left(x_{1}\right)=\left\{T x_{1} ; T \in \operatorname{Isom}(X)\right\}
$$

is a dense subset of $S_{X}$, there exists $T_{1} \in \operatorname{Isom}(X)$ such that $\left\|T_{1} x_{1}-x_{1}\right\|<\frac{\varepsilon}{4}$. Hence,

$$
\left\|T_{1} x_{i}-x_{i}\right\| \geqslant \varepsilon
$$

for some $1<i \leqslant n$. Let $T_{1}, \ldots, T_{n} \in \operatorname{Isom}(X)$ such that $\left\|T_{i} x_{1}-x_{i}\right\|<\frac{\varepsilon}{4}$. Then,

$$
\left\|T_{i} T_{1} x_{1}-x_{i}\right\| \leqslant\left\|T_{i} T_{1} x_{1}-T_{i} x_{1}\right\|+\left\|T_{i} x_{1}-x_{i}\right\|<\frac{\varepsilon}{2} .
$$

If $\operatorname{Isom}(X)$ was abelian, we would have for every $1 \leqslant i \leqslant n$,

$$
\left\|T_{1} x_{i}-x_{i}\right\| \leqslant\left\|T_{1} x_{i}-T_{1} T_{i} x_{1}\right\|+\left\|T_{i} T_{1} x_{1}-x_{i}\right\|<\varepsilon,
$$

which contradicts 4.2 . 
Proposition 4.16. If $\operatorname{Isom}(X)$ is SOT-discrete and acts almost-transitively in $S_{X}$, then $\operatorname{Isom}(X)$ is not abelian.

Proof. It follows from Lemma 4.15 and the fact that if $G$ is an SOT-discrete group, then $X$ has a distinguished family $\left\{x_{1}, \ldots, x_{n}\right\}$ for $G$, with $n \geqslant 1$. This implies also that $X$ has a distinguished family $\left\{x_{1}, \ldots, x_{n}\right\}$ for $G$ with $n \geqslant 2$, because if $F$ is distinguished for $G$ then $F \cup\{x\}$ is also distinguished for any $x \in X$.

\section{Questions and comments}

The proof of Proposition 4.11 is a generalization of an initial result of Ferenczi, Grivaux and Rosendal for $r=2$.

The examples presented show that there is no general relation between closed subspaces and their respective isometry groups, in terms of being light, apart from Proposition 4.8, Indeed:

1. $c_{0}$ is a closed subspace of $c, c_{0}$ is light, but $c$ is not;

2. $c$ is isometrically isomorphic to a closed subspace of $C[0,1], c$ is not light but $C[0,1]$ is light.

In view of Proposition 4.16, we may restate Question 4.14 in the following way:

Question 4.17. Does there exist a Banach space $X$ such that $\operatorname{Isom}(X)$ abelian, SOTdiscrete but whose orbit for some $x_{0} \in S_{X}$ is dense in $S_{X}$ ?

Finally, whether the converse to Megreleshvili's result holds remains an open question:

Question 4.18. Does a Banach space $X$ have the PCP if and only if all bounded subgroups of $G L(X)$ are light?

The answer is positive when $X$ has an unconditional basis: this follows from the following facts:

1. if $X$ is a separable Banach space containing an isomorphic copy of $c_{0}$, then $G L(X)$ contains a non-light bounded subgroup $G$;

2. an unconditional basis whose span does not contain $c_{0}$ must be boundedly complete;

3. separable dual spaces have the RNP and therefore the PCP. 
For further references, we reproduce below the classification of light groups that were cited in this thesis:

\begin{tabular}{|c|c|c|}
\hline Group or property & $\begin{array}{l}\text { Light/non- } \\
\text { light }\end{array}$ & Reference \\
\hline$\overline{G \leqslant G L(X) \text { bounded, } X \text { reflexive }}$ & Light & Meg01 \\
\hline$G \leqslant G L(X)$ bounded, $X$ separable dual space & Light & Meg01 \\
\hline $\operatorname{Isom}\left(C[-1,1]^{2}\right)$ & Non-light & Meg01 \\
\hline $\begin{array}{l}\text { Isom }(C(K)) \text {, if } K \text { contains an } n \text {-dimensional } \\
\text { region, } n \geqslant 2\end{array}$ & Non-light & AFGR17] \\
\hline $\operatorname{Isom}\left(\ell_{\infty}\right)$ & Non-light & Proposition 1.10 \\
\hline $\operatorname{Isom}\left(L_{1}[0,1]\right)$ & Non-light & Proposition 1.13 \\
\hline $\operatorname{Isom}\left(C\left(\{0,1\}^{\mathbb{N}}\right)\right)$ & Non-light & Proposition 1.11 \\
\hline SOT-compact groups & Light & Proposition 1.19 \\
\hline $\operatorname{Isom}(C[0,1] \times F), F$ finite & Light & Proposition 1.16 \\
\hline $\operatorname{Isom}\left(C_{0}(\mathbb{R})\right)$ & Light & Proposition 1.18 \\
\hline $\operatorname{Isom}\left(X_{\mathcal{S}_{n}}\right), n \in \mathbb{N}$ & Light & Proposition 2.7 \\
\hline $\begin{array}{l}G \leqslant G L(X) \text {, if } X \text { admits a } G \text {-invariant dense } \\
\text { LUR renorming }\end{array}$ & Light & Theorem 3.3 \\
\hline $\operatorname{Isom}\left(c_{0}\right)$ & Light & Proposition 3.5 \\
\hline $\begin{array}{l}G \leqslant G L(X) \text {, if } G \text { has a distinguished point but } \\
\text { has dual action SOT-indiscrete }\end{array}$ & Non-light & Theorem 4.4 \\
\hline $\operatorname{Isom}(c)$ & Non-light & Corollary 4.7 \\
\hline
\end{tabular}

Table 1 - Light/non-light groups 



\section{Polyhedrality of combinatorial spaces}

Let $X$ be a topological vector space and let $A \subset X$. We say that $\mathrm{A}$ is a convex polytope if

$$
A=\overline{\mathrm{co}}(F),
$$

for some finite subset $F \subset X$, where $\overline{\mathrm{co}}(F)$ denotes the closed convex hull of $F$. We say that a finite dimensional vector space $X$ is polyhedral if its closed unit ball $B_{X}$ is a convex polytope.

The concept of polyhedrality has many applications for finite dimensional spaces, which led several authors to expand this definition for infinite dimensional spaces. Fonf and Viselý collect in [FV04] eight of these definitions:

(I) $\left(\operatorname{ext} B_{X^{*}}\right)^{\prime} \subset\{0\}$, where

$$
A^{\prime}=\left\{f \in X^{*}: f \in \overline{A \backslash\{f\}}^{w^{*}}\right\}, \quad A \subset X^{*}
$$

(II) $\left(\operatorname{ext} B_{X^{*}}\right)^{\prime} \subset r B_{X^{*}}$, for some $0<r<1$;

(III) $\left(\operatorname{ext} B_{X^{*}}\right)^{\prime} \subset B_{X^{*}}^{0}$, where $B_{X}^{0}$ is the open unit ball of $X$;

(IV) $f(x)<1$, whenever $x \in S_{X}$ and $f \in\left(\operatorname{ext} B_{X^{*}}\right)^{\prime}$

(V) $\sup \left\{f(x): f \in \operatorname{ext} B_{X^{*}} \backslash D(x)\right\}<1$, for each $x \in S_{X}$, where

$$
D(x)=\left\{f \in S\left(X^{*}\right): f(x)=1\right\}
$$

(VI) every $x \in S_{X}$ has a neighborhood $V$ such that, for each $y \in V \cap S_{X}$, the segment $[x, y]$ lies entirely in $S_{X}$;

(VII) the set $M_{v}=\left\{x \in S_{X}: \max \langle D(x), v\rangle \leqslant 0\right\}$ is open in $S_{X}$ for each direction $v \in S_{X}$;

(VIII) the unit ball of every finite-dimensional subspace of $X$ is a polytope.

These definitions are non-equivalent. Indeed, Fonf and Viselý prove that

$$
(\mathrm{I}) \Longrightarrow(\mathrm{II}) \Longrightarrow(\mathrm{III}) \Longrightarrow(\mathrm{IV}) \Longrightarrow(\mathrm{V}) \Longrightarrow(\mathrm{VI}) \Longrightarrow(\mathrm{VII}) \Longrightarrow(\mathrm{VIII})
$$

and none of these implications can be reversed. The oldest and standard definition of polyhedrality is the definition (VIII), due to Klee, in [Kle60], motivated by the problem of existence of universal spaces for some classes of spaces, especially the existence of a universal space for the reflexive separable spaces (which was answered negatively by 
Szlenk later, in [Szl68]). Klee proved that $c_{0}$ is polyhedral and it is universal for all finite-dimensional polyhedral Banach spaces and asks whether there exists an infinitedimensional Banach space reflexive and polyhedral.

Lindenstrauss proved in [Lin66] that every infinite-dimensional space has a twodimensional quotient space whose unit ball is not a polygon. A consequence of this theorem is that no infinite-dimensional dual space (including reflexive spaces) is polyhedral. Indeed, if $X=Z^{*}$ is infinite dimensional, there exists $Y \leqslant Z$ such that $\operatorname{dim}(Z / Y)=2$ and $Z / Y$ is not polyhedral. Since $(Z / Y)^{*}$ is isometric to $Y^{\perp}=\left\{\varphi \in Z^{*}: \varphi(y)=0, \forall y \in Y\right\} \leqslant Z^{*}=X$ (see $\left[\mathrm{FHH}^{+} 11\right.$, Proposition 2.6]) and $\operatorname{dim}(Z / Y)^{*}=2$, it follows that $X$ is not polyhedral.

Recall that $B_{X}$ is weakly compact if $X$ is reflexive. Since the weak and the norm closures coincide in convex subsets of normed spaces, the Krein-Milman Theorem implies that

$$
B_{X}=\overline{\operatorname{co}}\left(\operatorname{ext} B_{X}\right)
$$

if $X$ is reflexive. This and the previous observation led Lindenstrauss to ask the following question:

Question 5.1 (Lindenstrauss, Lin66]). Does there exist a polyhedral infinite-dimensional Banach space whose unit ball is the closed convex hull of its extreme points?

Obviously $c_{0}$ is not a solution for this question, since it has no extreme points, although it is polyhedral. However, De Bernardi gave in [DB17] the first solution for Lindenstrauss's problem, using a renorming of $c_{0}$. The construction is the following: let $\left(\gamma_{n}\right) \in c_{0}$, with $0<\gamma_{n}<\frac{1}{2}$ for every $n$ and let $\delta_{n}=2 \gamma_{n}$. For each $n \in \mathbb{N}$, let

$$
A_{n}=\left\{\left(1-\delta_{n}\right) \varepsilon_{1} e_{1}+\cdots+\left(1-\delta_{n}\right) \varepsilon_{n} e_{n}+\left(1+\gamma_{n}\right) \varepsilon_{n+1} e_{n+1}: \varepsilon_{1}, \ldots, \varepsilon_{n+1} \in\{ \pm 1\}\right\},
$$

where $e_{1}, e_{2}, \ldots$ is the usual basis of $c_{0}$. Put $A=\bigcup_{n=1}^{\infty} A_{n}$ and $B=\overline{\operatorname{co}}(A)$. Since $\left(\gamma_{n}\right) \in c_{0}$, then $\left\{ \pm e_{1} \pm e_{2} \pm \cdots \pm e_{n}: n \in \mathbb{N}\right\} \subset B$, which implies that $B_{c_{0}} \subset B$. Since $A$ is bounded, $B$ is the closed unit ball of an equivalent norm $\|\cdot\|$. Define $X=\left(c_{0},\|\cdot\|\right)$. De Bernardi proved that $X$ is $(V)$-polyhedral, i.e., it satisfies the fifth definition of [FV04], which is stronger than Klee's definition, and also showed that $\overline{\operatorname{co}}\left(\operatorname{ext}\left(B_{X}\right)\right)=B_{X}$.

The purpose of this chapter is to provide new examples of spaces that solve Question 5.1. We will prove in Theorem 5.12 that every combinatorial space $X_{\mathcal{F}}$ is $(\mathrm{V})$ polyhedral. In particular, the Schreier spaces of countable order $X_{\mathcal{S}_{\alpha}}$ are new solutions to Lindenstrauss's problem. We also prove (Corollary 5.10) that the dual space of every combinatorial space has the convex series representation property (CSRP), i.e., every point of the unit ball of $X_{\mathcal{F}}^{*}$ can be expressed as an infinite convex combination of extreme points of $X_{\mathcal{F}}^{*}$. 


\section{$5.1 \lambda$-property and CSRP}

The $\lambda$-property was defined by Aron and Lohman in [AL87] as follows: let $X$ be a normed space and let $x \in B_{X}$. If $e \in \operatorname{ext}\left(B_{X}\right),\|y\| \leqslant 1,0<\lambda<1$ and $x=\lambda e+(1-\lambda) y$ we say the ordered triple $(e, y, \lambda)$ is amenable to $x$. In this case, we define

$$
\lambda(x)=\sup \{\lambda:(e, y, \lambda) \text { is amenable to } x\}
$$

(the function $\lambda$ measures how close a point of the ball is close to being an extreme point of the ball). A Banach space $X$ is said to have the $\lambda$-property if each $x \in B_{X}$ admits an amenable triple. If $X$ has the $\lambda$-property and, in addition, satisfies

$$
\inf \left\{\lambda(x): x \in B_{X}\right\}>0
$$

we say $\mathrm{X}$ has the uniform $\lambda$-property.

Aron and Lohman prove that many classical Banach spaces have the $\lambda$-property or the uniform $\lambda$-property. For example, they prove that every finite dimensional normed space has the uniform $\lambda$-property. Also, if $X$ is a strictly convex normed space, $T$ is a compact metric space and $C_{X}(T)$ is the space of continuous $X$-valued functions on $T$ endowed with the sup norm, then:

1. $C_{X}(T)$ has the uniform $\lambda$-property, if $X$ is an infinite dimensional space;

2. $C_{X}[0,1]$ has the uniform $\lambda$-property, if $X$ is a real space with $\operatorname{dim} X \geqslant 2$;

3. $C_{X}[0,1]$ does not have the $\lambda$-property, if $X$ is a real space with $\operatorname{dim} X=1$;

4. $\ell_{1}(X)$ has the $\lambda$-property but not the uniform $\lambda$-property;

5. $\ell_{\infty}(X)$ has the uniform $\lambda$-property;

6. $c(X)$ has the uniform $\lambda$-property, for $X$ infinite-dimensional;

Among other useful geometric implications for these properties, the authors prove the following:

Theorem 5.2 ([AL87, Theorem 3.3). Let $X$ be a Banach space satisfying the $\lambda$-property. Then,

$$
B_{X}=\overline{\operatorname{co}}\left(\operatorname{ext}\left(B_{X}\right)\right)
$$

They also observe that if $X$ has the uniform $\lambda$-property, then each $x \in B_{X}$ admits an expansion as an infinite convex combination of members of $\operatorname{ext}\left(B_{X}\right)$, i.e., there exist 
a sequence of positive numbers $\left(\lambda_{k}\right)$ with $\sum_{k=1}^{\infty} \lambda_{k}=1$ and a sequence of extreme points $\left(e_{k}\right)$ of $B_{X}$ such that

$$
x=\sum_{k=1}^{\infty} \lambda_{k} e_{k} .
$$

This property is called convex series representation property $(C S R P$ in [Loh89]. On the other hand, it is not hard to see that the CSRP implies the uniform $\lambda$-property. Actually, in ALS91 the authors prove that the CSRP implies the $\lambda$-property and, hence, both properties are equivalent.

In [ST90], Shura and Trautman prove that the Schreier space $X_{\mathcal{S}_{1}}$ has the $\lambda$ property. Beanland and Chu extended this result in $\mathrm{ABC19}$ :

Theorem 5.3 (Theorem 3.1, [ABC19]). Let $\alpha$ be a non-zero countable ordinal. Then:

1. The space $X_{\mathcal{S}_{\alpha}}$ has the $\lambda$-property.

2. For $p \in(1, \infty)$, the space $X_{\mathcal{S}_{\alpha}}^{p}$ has the uniform $\lambda$-property, where $X_{\mathcal{S}_{\alpha}}^{p}$ denotes the p-convexification of $X_{\mathcal{S}_{\alpha}}$, i.e., the completion of $c_{00}$ under the norm

$$
\|x\|_{X_{\mathcal{S}_{\alpha}}^{p}}=\sup _{F \in \mathcal{S}_{\alpha}}\left(\sum_{i \in F}\left|x_{i}\right|^{p}\right)^{\frac{1}{p}}
$$

By Theorem 5.2, it follows that:

Corollary 5.4. For every non-zero countable ordinal $\alpha$,

$$
B_{X_{\mathcal{S}_{\alpha}}}=\overline{\operatorname{co}}\left(\operatorname{ext}\left(B_{X_{\mathcal{S}_{\alpha}}}\right)\right)
$$

Hence, the Schreier spaces of countable order are possible candidates to give a positive answer to 5.1, different than the one provided by De Bernardi. In fact, in Theorem 5.12 we will prove that every combinatorial space is (V)-polyhedral.

\subsection{Characterization of the dual of combinatorial spaces}

We will prove in Theorem 5.12 that for every regular family $\mathcal{F}$ the combinatorial space $X_{\mathcal{F}}$ is a $(V)$-polyhedral space. In order to prove Theorem 5.12 we need a characterization for the extreme points of the dual of $X_{\mathcal{F}}$. Gowers [Gow09] states in his blog the following:

(...) a long time ago I proved a result I quite liked, motivated by the question of showing that explicit spaces contained $c_{0}$ or $\ell_{p}$. However, the result isn't quite what you would expect, because it includes a lot of spaces that aren't even definable. Let us call a space combinatorial if it is defined by means of a system $\mathcal{A}$ of finite sets in the following simple way: $\|x\|=\sup _{A \in \mathcal{A}} \sum_{n \in A}\left|x_{n}\right|$. I showed that every combinatorial space 
contains $c_{0}$ or $\ell_{1}$. (We need to assume that $\mathcal{A}$ contains all singletons just to make sure we've got a norm and without loss of generality if $A \in \mathcal{A}$ and $B \subset A$ then $B \in \mathcal{A}$.) I also generalized this observation somewhat. I never published the result, because I gave a talk about it at a conference and someone pointed out to me that it was known that spaces for which the unit ball of the dual space has only countably many extreme points must contain $c_{0}$. My result was an easy consequence of that: if you have a nested sequence $A_{1} \subset A_{2} \subset \ldots$ of sets in $\mathcal{A}$, then the basis vectors in the union of the $A_{i}$ generate $\ell_{1}$, and if you don't have such a sequence, then every element of $\mathcal{A}$ is a subset of some maximal element $A$, and all the extreme points of the dual space are sequences that take the value \pm 1 on a maximal set and 0 outside it. So I had proved (by a similar method) a special case of a known result.

In Theorem 5.9 we will give a proof for this characterization of $\operatorname{ext}\left(B_{X_{\mathcal{F}}^{*}}\right)$ stated by Gowers but before that we will provide a characterization of the whole unit ball $B_{X_{\mathcal{F}}^{*}}$. It is a well-known fact that the unit ball of dual spaces can be characterized by norming sets. A set $\Lambda \subset B_{X^{*}}$ is called a norming set for $X$ if

$$
\|x\|=\sup _{\varphi \in \Lambda}|\varphi(x)|
$$

for every $x \in X$.

Proposition 5.5. $\Lambda \subset B_{X^{*}}$ is a norming set for $X$ if, and only if,

$$
B_{X^{*}}=\overline{\mathrm{co}}^{w *}(\Lambda)
$$

where $\overline{\mathrm{co}}^{w *}(\Lambda)$ denotes the weak*-closed convex hull of $\Lambda$.

Proof. Suppose that $\Lambda \subset B_{X^{*}}$ is a norming set for $X$ and suppose by contradiction that $B_{X^{*}} \neq \overline{\mathrm{CO}}^{w *}(\Lambda)$. Let $f \in B_{X^{*}} \backslash \overline{\mathrm{CO}}^{w *}(\Lambda)$. By the Hahn-Banach separation theorem, there exists $\Phi \in\left(X^{*}, w^{*}\right)^{*}$ such that

$$
\sup \left\{|\Phi(g)|: g \in \overline{\mathrm{cO}}^{w *}(\Lambda)\right\}<\Phi(f) .
$$

Since $\left(X^{*}, w^{*}\right)^{*}$ can be identified with $X$ (see [ $\mathrm{FHH}^{+} 11$, Proposition 3.22]), there exists $x \in X$ such that

$$
\sup \left\{|g(x)|: g \in \overline{\mathrm{co}}^{w *}(\Lambda)\right\}<f(x)=|f(x)| \leqslant\|f\| \cdot\|x\|=\|x\|,
$$

which contradicts the fact that $\Lambda$ is a norming set. On the other hand, suppose that $B_{X^{*}}=\overline{\mathrm{CO}}^{w *}(\Lambda)$ and suppose that $\Lambda$ is not a norming set. Then, there exists $x \in X$ such that

$$
\sup _{\varphi \in \Lambda}|\varphi(x)|<\|x\| .
$$

Hence, for every $\varphi_{1}, \ldots, \varphi_{n} \in \Lambda$ and every $a_{1}, \ldots, a_{n} \geqslant 0$, with $\sum_{i=1}^{n} a_{i}=1$, we have

$$
\left|\sum_{i=1}^{n} a_{i} \varphi_{i}(x)\right| \leqslant \sum_{i=1}^{n} a_{i}\left|\varphi_{i}(x)\right| \leqslant \sup _{\varphi \in \Lambda}|\varphi(x)|
$$


and therefore

$$
\sup _{\psi \in \operatorname{co}(\Lambda)}|\psi(x)|<\|x\|=\max _{\xi \in B_{X^{*}}}|\xi(x)|
$$

which contradicts the $w^{*}$-density of $\operatorname{co}(\Lambda)$ in $B_{X^{*}}$.

We will use Proposition 5.5 to give a characterization of the ball of dual of combinatorial spaces:

Theorem 5.6. Let $X_{\mathcal{F}}$ be a combinatorial space and let

$$
W_{\mathcal{F}}=\left\{g=\sum_{k \in F} \pm e_{k}^{*}: F \in \mathcal{F}\right\} .
$$

Then,

$$
B_{X_{\mathcal{F}}^{*}}=\left\{f=\sum_{i=1}^{\infty} \lambda_{i} f_{i}: f_{i} \in W_{\mathcal{F}}, \lambda_{i} \geqslant 0 \text { and } \sum_{i=1}^{\infty} \lambda_{i} \leqslant 1\right\} .
$$

Proof. Notice that for every $x \in X_{\mathcal{F}} \cap c_{00}$ we have

$$
\|x\|_{X_{\mathcal{F}}}=\sup _{F \in \mathcal{F}} \sum_{k \in F}\left|x_{k}\right|=\sup _{f \in W_{\mathcal{F}}}|f(x)|
$$

and by density of $c_{00}$ in $X_{\mathcal{F}}$ the same holds for any $x \in X_{\mathcal{F}}$, i.e., $W_{\mathcal{F}}$ is a norming set for $X_{\mathcal{F}}$. By Proposition 5.5 it follows that

$$
B_{X_{\mathcal{F}}^{*}}=\overline{\operatorname{co}}^{w *}\left(W_{\mathcal{F}}\right) .
$$

Thus, we need to show that

$$
\overline{\mathrm{CO}}^{w *}\left(W_{\mathcal{F}}\right)=\left\{f=\sum_{i=1}^{\infty} \lambda_{i} f_{i}: f_{i} \in W_{\mathcal{F}}, \lambda_{i} \geqslant 0 \text { and } \sum_{i=1}^{\infty} \lambda_{i} \leqslant 1\right\} .
$$

Let $f \in \overline{\mathrm{co}}^{w *}\left(W_{\mathcal{F}}\right)$ and let $\left(f_{n}\right)_{n=1}^{\infty}$ be a sequence in $\operatorname{co}\left(W_{\mathcal{F}}\right)$ such that

$$
f_{n} \stackrel{w *}{\longrightarrow} f
$$

i.e., $f_{n}$ converges pointwise to $f$. Each $f_{n}$ can be written as

$$
f_{n}=\sum_{m=1}^{d_{n}} \lambda_{n, m} f_{n, m}
$$

with $\lambda_{n, m} \geqslant 0, \sum_{m=1}^{d_{n}} \lambda_{n, m}=1, d_{n} \geqslant 1$ and $f_{n, m} \in W_{\mathcal{F}}$. Also, each $f_{n, m}$ has the form

$$
f_{n, m}=\sum_{k \in F_{n, m}} \varepsilon_{n, m}^{(k)} e_{k}^{*}
$$

where $F_{n, m}=\left\{k_{1}<k_{2}<\cdots<k_{t}\right\} \in \mathcal{F}$ and $\varepsilon_{n, m}=\left(\varepsilon_{n, m}^{\left(k_{1}\right)}, \ldots, \varepsilon_{n, m}^{\left(k_{t}\right)}\right) \in\{-1,1\}^{t}$. Then, 


$$
\begin{aligned}
f_{1} & =\lambda_{1,1} \sum_{k \in F_{1,1}} \varepsilon_{1,1}^{(k)} e_{k}^{*}+\lambda_{1,2} \sum_{k \in F_{1,2}} \varepsilon_{1,2}^{(k)} e_{k}^{*}+\cdots+\lambda_{1, d_{1}} \sum_{k \in F_{1, d_{1}}} \varepsilon_{1, d_{1}}^{(k)} e_{k}^{*} \\
f_{2} & =\lambda_{2,1} \sum_{k \in F_{2,1}} \varepsilon_{2,1}^{(k)} e_{k}^{*}+\lambda_{2,2} \sum_{k \in F_{2,2}} \varepsilon_{2,2}^{(k)} e_{k}^{*}+\cdots+\lambda_{2, d_{2}} \sum_{k \in F_{2, d_{2}}} \varepsilon_{2, d_{2}}^{(k)} e_{k}^{*} \\
& \vdots \\
f_{n} & =\lambda_{n, 1} \sum_{k \in F_{n, 1}} \varepsilon_{n, 1}^{(k)} e_{k}^{*}+\lambda_{n, 2} \sum_{k \in F_{n, 2}} \varepsilon_{n, 2}^{(k)} e_{k}^{*}+\cdots+\lambda_{n, d_{n}} \sum_{k \in F_{n, d_{n}}} \varepsilon_{n, d_{n}}^{(k)} e_{k}^{*} \\
& \vdots
\end{aligned}
$$

We want to show that

$$
f=w^{*} \lim _{n \rightarrow \infty} f_{n}=\lambda_{1} \sum_{k \in F_{1}} \varepsilon_{1}^{(k)} e_{k}^{*}+\lambda_{2} \sum_{k \in F_{2}} \varepsilon_{2}^{(k)} e_{k}^{*}+\lambda_{3} \sum_{k \in F_{3}} \varepsilon_{3}^{(k)} e_{k}^{*}+\ldots
$$

for some $\lambda_{i}>0, F_{i} \in \mathcal{F}, \varepsilon_{i}^{(k)} \in\{-1,1\}$ for every $i \in \mathbb{N}$ and $k \in F_{i}$ and $\sum_{i=1}^{\infty} \lambda_{i} \leqslant 1$.

Let

$$
\mathcal{N}=\left\{t \in \mathbb{N} ; f\left(e_{t}\right) \neq 0\right\}
$$

and denote by $\mathcal{P}$ the class of pairs

$$
\mathcal{P}=\left\{(F, \varepsilon): F=\left\{k_{1}<k_{2}<\cdots<k_{j}\right\} \in \mathcal{F} \text { and } \varepsilon=\left(\varepsilon^{\left(k_{1}\right)}, \varepsilon^{\left(k_{2}\right)}, \ldots, \varepsilon^{\left(k_{j}\right)}\right) \in\{-1,1\}^{j}\right\}
$$

In $\mathcal{P}$, we will say that $(G, \delta)$ is an extension of $(F, \varepsilon)$ and we will denote

$$
(F, \varepsilon) \sqsubseteq(G, \delta)
$$

if $F=\left\{k_{1}<\cdots<k_{i}\right\} \subseteq G=\left\{l_{1}<\cdots<l_{j}\right\}$ and $\varepsilon^{\left(k_{t}\right)}=\delta^{\left(k_{t}\right)}$ for every $k_{t} \in F$.

For each $j \in \mathbb{N}$, define $\mathcal{P}_{j}$ to be the class of pairs $(F, \varepsilon)$ of $\mathcal{P}$ such that:

1. $F \subset \mathcal{N}$

2. $\operatorname{card}(F)=j$;

3. there exists the limit $\mu_{(F, \varepsilon)}=\lim _{n \rightarrow \infty}\left(\sum_{\substack{1 \leqslant m \leqslant d_{n} \\(F, \varepsilon) \subseteq\left(F_{n, m}, \varepsilon_{n, m}\right)}} \lambda_{n, m}\right)$;

4. $\mu_{(F, \varepsilon)}>0$.

We will say that $\left(F_{n, m}, \varepsilon_{n, m}\right)$ is a maximal extension of $(F, \varepsilon)$ and we will denote

$$
(F, \varepsilon) \stackrel{\max }{\sqsubseteq}\left(F_{n, m}, \varepsilon_{n, m}\right)
$$

if: 
1. $(F, \varepsilon) \sqsubseteq\left(F_{n, m}, \varepsilon_{n, m}\right)$;

2. $(F, \varepsilon) \in \mathcal{P}_{j} \Longrightarrow(G, \delta) \nsubseteq\left(F_{n, m}, \varepsilon_{n, m}\right)$, for any $(G, \delta) \in \mathcal{P}_{j+1}$.

The elements $(F, \varepsilon) \in \bigcup_{j=1}^{\infty} \mathcal{P}_{j}$ will be used to define the sets $F_{i}$ and the signs $\varepsilon_{i}^{(k)}$ in (5.1). It still remains to define the coefficients $\lambda_{i}$. This will be done as follows: let $\left(F_{i}, \varepsilon_{i}\right)_{i=1}^{\infty}$ be an enumeration of $\bigcup_{i=1}^{\infty} \mathcal{P}_{i}$. Let

$$
\lambda_{\left(F_{1}, \varepsilon_{1}\right)}=\limsup _{n}\left(\sum_{\substack{1 \leqslant m \leqslant d_{n} \\(F, \varepsilon) \stackrel{\text { max }}{\sqsubseteq}\left(F_{n, m}, \varepsilon_{n, m}\right)}} \lambda_{n, m}\right)=\lim _{\substack{n \rightarrow \infty \\ n \in N_{1}}}\left(\sum_{\substack{1 \leqslant m \leqslant d_{n} \\(F, \varepsilon) \stackrel{\text { max }}{\sqsubseteq}\left(F_{n, m}, \varepsilon_{n, m}\right)}} \lambda_{n, m}\right),
$$

where $N_{1}$ is an infinite subset of $\mathbb{N}$. In the same way we define $\lambda_{\left(F_{2}, \varepsilon_{2}\right)}, \lambda_{\left(F_{2}, \varepsilon_{2}\right)}, \ldots$, taking infinite subsets $N_{2}, N_{3}, \ldots$ of $\mathbb{N}$ such that

$$
N_{1} \supseteq N_{2} \supseteq N_{3} \supseteq \cdots
$$

Notice that

$$
\sum_{i=1}^{\infty} \lambda_{\left(F_{i}, \varepsilon_{i}\right)} \leqslant 1
$$

Indeed, if $\sum_{i=1}^{\infty} \lambda_{\left(F_{i}, \varepsilon_{i}\right)}>1$, there would exist $r \in \mathbb{N}$ such that $\sum_{i=1}^{r} \lambda_{(F, \varepsilon)}>1$. Hence, for sufficiently large $n \in N_{r}, \sum_{1 \leqslant m \leqslant d_{n}} \lambda_{n, m}>1$, since each $\left(F_{n, m}, \varepsilon_{n, m}\right)$ is a maximal extension of at most one pair $\left(F_{i}, \varepsilon_{i}\right)$, which contradicts the fact that $\sum_{m=1}^{d_{n}} \lambda_{n, m}=1$.

For each $i \in \mathbb{N}$ define

$$
g_{\left(F_{i}, \varepsilon_{i}\right)}=\sum_{k \in F_{i}} \varepsilon_{i}^{(k)} e_{k}^{*}
$$

Let

$$
g=\sum_{i=1}^{\infty} \lambda_{\left(F_{i}, \varepsilon_{i}\right)} g_{\left(F_{i}, \varepsilon_{i}\right)} .
$$

We claim that $f\left(e_{t}\right)=g\left(e_{t}\right)$ for every $t \in \mathcal{N}$ and hence

$$
f=g \text {. }
$$

Indeed, suppose by contradiction that $f\left(e_{t}\right)=M$ and $g\left(e_{t}\right)=L$, with $M \neq L$, for some $t \in \mathcal{N}$. Let

$$
M^{+}=\limsup _{n}\left(\sum_{\substack{1 \leqslant m \leqslant d_{n} \\ t \in F_{n, m} \\ \varepsilon_{n, m}^{(t)}=1}} \lambda_{n, m}\right)=\lim _{\substack{n \rightarrow \infty \\ n \in N^{\prime}}}\left(\sum_{\substack{1 \leqslant m \leqslant d_{n} \\ t \in F_{n, m} \\ \varepsilon_{n, m}^{(t)}=1}} \lambda_{n, m}\right),
$$

where $N^{\prime}$ is an infinite subset of $\mathbb{N}$, and let

$$
M^{-}=M-M^{+}=\lim _{\substack{n \rightarrow \infty \\ n \in N^{\prime}}}\left(\sum_{\substack{1 \leqslant m \leqslant d_{n} \\ t \in F_{n} \\ \varepsilon_{n, m}^{(t)}=-1}} \lambda_{n, m}\right)
$$


Hence,

$$
f\left(e_{t}\right)=\lim _{n \rightarrow \infty} f_{n}\left(e_{t}\right)=\lim _{n \rightarrow \infty}\left(\sum_{\substack{1 \leqslant m \leqslant d_{n} \\ t \in F_{n, m}}} \lambda_{n, m} \varepsilon_{n, m}^{(t)}\right)=M^{+}-M^{-} .
$$

On the other hand,

$$
g\left(e_{t}\right)=\sum_{i=1}^{\infty} \lambda_{\left(F_{i}, \varepsilon_{i}\right)} g_{\left(F_{i}, \varepsilon_{i}\right)}\left(e_{t}\right)=\sum_{\substack{1 \leqslant i<\infty \\ t \in F_{i}}} \lambda_{\left(F_{i}, \varepsilon_{i}\right)} \varepsilon_{i}^{(t)}=L^{+}-L^{-},
$$

where $L^{+}=\sum_{\substack{1 \leqslant i<\infty \\ t \in F_{i} \\ \varepsilon_{i}^{(t)}=1}} \lambda_{\left(F_{i}, \varepsilon_{i}\right)}$ and $L^{-}=\sum_{\substack{1 \leqslant i<\infty \\ t \in F_{i} \\ \varepsilon_{i}^{(t)}=-1}} \lambda_{\left(F_{i}, \varepsilon_{i}\right)}$.

Without loss of generality, suppose that $M^{+} \neq L^{+}$and let

$$
\left|L^{+}-M^{+}\right|=\zeta>0 \text {. }
$$

For sufficiently large $r \in \mathbb{N}$, we have

$$
\left|L^{+}-\sum_{\substack{1 \leqslant i \leqslant r \\ t \in F_{i} \\ \varepsilon_{i}^{(t)}=1}} \lambda_{\left(F_{i}, \varepsilon_{i}\right)}\right|<\frac{\zeta}{4}
$$

Also, for sufficiently large $n \in N^{\prime}$,

$$
\left|\sum_{\substack{1 \leqslant i \leqslant r \\ t \in F_{i} \\ \varepsilon_{i}^{(t)}=1}} \lambda_{\left(F_{i}, \varepsilon_{i}\right)}-\sum_{\substack{1 \leqslant i \leqslant r \\ 1 \leqslant m \leqslant d_{n} \\ \max }} \lambda_{n, m}\right|<\frac{\zeta}{4} .
$$

Hence,

$$
\begin{aligned}
& \left|L^{+}-\quad \sum_{\substack{1 \leqslant i \leqslant r \\
1 \leqslant m \leqslant d_{n} \\
\max }} \lambda_{n, m}\right|<\frac{\zeta}{2} \\
& \left(F_{i}, \varepsilon_{i}\right) \stackrel{\max }{\sqsubseteq}\left(F_{n, m}, \varepsilon_{n, m}\right)
\end{aligned}
$$

and then

$$
\left|M^{+}-\sum_{\substack{1 \leqslant i \leqslant r \\ 1 \leqslant m \leqslant d_{n} \\ \max }} \lambda_{n, m}\right| \geqslant \frac{\zeta}{2},
$$

i.e.,

$$
\left|\lim _{\substack{k \rightarrow \infty \\ k \in N^{\prime}}}\left(\sum_{\substack{1 \leqslant l \leqslant d_{k} \\ t \in F_{k, l} \\ \varepsilon_{k, l}^{(t)}=1}} \lambda_{k, l}\right)-\sum_{\substack{1 \leqslant i \leqslant r \\ 1 \leqslant m \leqslant d_{n} \\ \max \left(F_{i}, \varepsilon_{i}\right) \stackrel{(5}{\sqsubseteq}\left(F_{n, m}, \varepsilon_{n, m}\right)}} \lambda_{n, m}\right| \geqslant \frac{\zeta}{2},
$$

for every $r \in \mathbb{N}$ and $n \in N^{\prime}$ sufficiently large. It means that for every $k$ sufficiently large we can find sets $F_{k, l_{1}}, \ldots, F_{k, l_{u}}$ such that $t \in F_{k, l_{j}}, \varepsilon_{k, l_{j}}^{(t)}=1$ and $\sum_{j=1}^{u} \lambda_{k, l_{j}} \geqslant \frac{\zeta}{2}$, which are not maximal extensions of any $\left(F_{i}, \varepsilon_{i}\right)$. This is a contradiction, because in the worst case they would be a maximal extension of $(\{t\}, 1)$. 
Notice that

$$
W_{\mathcal{F}^{\max }}=\left\{g=\sum_{k \in F} \pm e_{k}^{*}: F \in \mathcal{F}^{\max }\right\} .
$$

is also a norming set for $X_{\mathcal{F}}^{*}$ and hence $B_{X_{\mathcal{F}}^{*}}=\overline{\mathrm{CO}}^{w *}\left(W_{\mathcal{F}^{\max }}\right)$. For this reason, we may ask:

Question 5.7. Can we replace $W_{\mathcal{F}}$ by $W_{\mathcal{F}^{\max }}$ in Theorem 5.6?

We will provide a positive answer to this question in next corollary. An important consequence of this result is that the dual of any combinatorial space has the CSRP, as we will see in Corollary 5.10.

Corollary 5.8. Let $X_{\mathcal{F}}$ be a combinatorial space. Then,

$$
B_{X_{\mathcal{F}}^{*}}=\left\{f=\sum_{i=1}^{\infty} \lambda_{i} f_{i}: f_{i} \in W_{\mathcal{F}^{\max }}, \lambda_{i} \geqslant 0 \text { and } \sum_{i=1}^{\infty} \lambda_{i} \leqslant 1\right\} .
$$

Proof. Let $f \in B_{X_{\mathcal{F}}^{*}}$. By Theorem $5.6 f$ can be written as

$$
f=\sum_{i=1}^{\infty} \lambda_{i} f_{i}
$$

with $F_{i} \in \mathcal{F}, \lambda_{i}>0, \sum_{i=1}^{\infty} \lambda_{i} \leqslant 1$ and

$$
f_{i}=\sum_{k \in F_{i}} \varepsilon_{i}^{(k)} e_{k}^{*}
$$

with $\varepsilon_{i}^{(k)} \in\{-1,1\}$. For each $i$, take $G_{i} \in \mathcal{F}$ such that $G_{i} \cap F_{i}=\emptyset$ and

$$
H_{i}=F_{i} \cup G_{i} \in \mathcal{F}^{\max }
$$

Then,

$$
f_{i}=\frac{1}{2}\left(\sum_{k \in F_{i}} \varepsilon_{i}^{(k)} e_{k}^{*}+\sum_{k \in G_{i}} e_{k}^{*}\right)+\frac{1}{2}\left(\sum_{k \in F_{i}} \varepsilon_{i}^{(k)} e_{k}^{*}+\sum_{k \in G_{i}}-e_{k}^{*}\right) .
$$

For each $i \in \mathbb{N}$, define

$$
\tilde{f}_{2 i-1}=\sum_{k \in F_{i}} \varepsilon_{i}^{(k)} e_{k}^{*}+\sum_{k \in G_{i}} e_{k}^{*}, \quad \tilde{f}_{2 i}=\sum_{k \in F_{i}} \varepsilon_{i}^{(k)} e_{k}^{*}+\sum_{k \in G_{i}}-e_{k}^{*}
$$

and

$$
\tilde{\lambda}_{2 i-1}=\tilde{\lambda}_{2 i}=\frac{\lambda_{i}}{2}
$$

Therefore,

$$
f=\sum_{i=1}^{\infty} \tilde{\lambda}_{i} \tilde{f}_{i}
$$

with $\tilde{\lambda}_{i} \geqslant 0, \sum_{i=1}^{\infty} \tilde{\lambda}_{i} \leqslant 1$ and $\tilde{f}_{i} \in W_{\mathcal{F}^{\max }}$ for every $i \in \mathbb{N}$.

Finally, we are able to prove Gowers's statement: 
Theorem 5.9. Let $X_{\mathcal{F}}$ be a combinatorial space. Then,

$$
\operatorname{ext}\left(B_{X_{\mathcal{F}}^{*}}\right)=\left\{\sum_{i \in F} \varepsilon_{i} e_{i}^{*}: F \in \mathcal{F}^{\max } \text { and } \varepsilon_{i} \in\{-1,1\}\right\} \text {. }
$$

Proof. Let $f \in \operatorname{ext}\left(B_{X_{\mathcal{F}}^{*}}\right)$ and suppose that $f \notin\left\{\sum_{i \in F} \varepsilon_{i} e_{i}^{*}: F \in \mathcal{F}^{\max }\right.$ and $\left.\varepsilon_{i} \in\{-1,1\}\right\}$. Notice that

$$
\|x\|_{X_{\mathcal{F}}}=\sup _{f \in W_{\mathcal{F}}}|f(x)| \quad \text { for every } x \in X_{\mathcal{F}}
$$

By Proposition 5.5, it follows that

$$
f \in \overline{\mathrm{CO}}^{w *}\left(W_{\mathcal{F}}\right)
$$

We will consider three cases:

1. If $f \in W_{\mathcal{F}}$, then $f=\sum_{i \in F} \varepsilon_{i} e_{i}^{*}$, with $F \in \mathcal{F} \backslash \mathcal{F}^{\text {max }}$ and $\varepsilon_{i} \in\{ \pm 1\}$ for every $i \in F$. Let $i_{0} \in \mathbb{N} \backslash F$ such that $F \cup\left\{i_{0}\right\} \in \mathcal{F}$. Notice that $f \pm e_{i_{0}}^{*} \in B_{X_{\mathcal{F}}^{*}}$ and

$$
f=\frac{1}{2}\left[\left(f+e_{i_{0}}^{*}\right)+\left(f-e_{i_{0}}^{*}\right)\right]
$$

which contradicts $f \in \operatorname{ext}\left(B_{X_{\mathcal{F}}^{*}}\right)$.

2. If $f \in \operatorname{co}\left(W_{\mathcal{F}}\right), f=\sum_{i=1}^{n} \lambda_{i} f_{i}$, with $f_{i} \in W_{\mathcal{F}}, f_{i}$ not all the same, $\lambda_{i}>0$ and $\sum_{i=1}^{n} \lambda_{i}=1$, then obviously $f \notin \operatorname{ext}\left(B_{X_{\mathcal{F}}^{*}}\right)$.

3. If $f \in \overline{\mathrm{co}}^{w *}\left(W_{\mathcal{F}}\right) \backslash \operatorname{co}\left(W_{\mathcal{F}}\right)$, by Theorem 5.6 $f=\sum_{i=1}^{\infty} \lambda_{i} f_{i}$, with $\lambda_{i}>0, \sum_{i=1}^{\infty} \lambda_{i} \leqslant 1$ and $f_{i} \in W_{\mathcal{F}}$ are not all the same. Then,

$$
f=\lambda_{1} f_{1}+\left(1-\lambda_{1}\right)\left(\frac{\lambda_{2}}{1-\lambda_{1}} f_{2}+\frac{\lambda_{3}}{1-\lambda_{1}} f_{3}+\ldots\right)
$$

and $\left(\frac{\lambda_{2}}{1-\lambda_{1}} f_{2}+\frac{\lambda_{3}}{1-\lambda_{1}} f_{3}+\ldots\right) \in B_{X_{\mathcal{F}}^{*}}$, since $\sum_{i=2}^{\infty} \frac{\lambda_{i}}{1-\lambda_{1}} \leqslant 1$. Hence, in this case we also have $f \notin \operatorname{ext}\left(B_{X_{\mathcal{F}}^{*}}\right)$.

This proves that $\operatorname{ext}\left(B_{X_{\mathcal{F}}^{*}}\right) \subseteq\left\{\sum_{i \in F} \varepsilon_{i} e_{i}^{*}: F \in \mathcal{F}^{\max }\right.$ and $\left.\varepsilon_{i} \in\{-1,1\}\right\}$. On the other hand, let $f=\sum_{i \in F} \varepsilon_{i} e_{i}^{*}$ with $F \in \mathcal{F}^{\text {max }}$ and $\varepsilon_{i} \in\{-1,1\}$. Suppose that $f \notin \operatorname{ext}\left(B_{X_{\mathcal{F}}^{*}}\right)$. Let $g, h \in S\left(X_{\mathcal{F}}^{*}\right)$ such that $g \neq h$ and $f=\frac{g+h}{2}$. Notice that

$$
g\left(e_{i}\right)=h\left(e_{i}\right)=f\left(e_{i}\right) \text {, for every } i \in F .
$$

Indeed, if we had, for example, $\varepsilon_{i}=1$ and $g\left(e_{i}\right)>h\left(e_{i}\right)$ for some $i \in F$, then

$$
f\left(e_{i}\right)=1=\frac{g\left(e_{i}\right)+h\left(e_{i}\right)}{2} \Longrightarrow g\left(e_{i}\right)>1 \Longrightarrow g \notin S\left(X_{\mathcal{F}}^{*}\right) .
$$


Suppose now that $g\left(e_{i_{0}}\right) \neq 0$ for some $i_{0} \notin F$. Let $x=\sum_{i \in F} a_{i} e_{i}$ such that $f(x)=\sum_{i \in F}\left|a_{i}\right|=1$ and $\left|a_{i}\right| \neq 0$ for every $i \in F$. Let $\eta=\min \left\{\left|a_{i}\right| ; i \in F\right\}$ and let $y=x+\frac{\eta}{2} e_{i_{0}}$. Notice that $f(y)=f(x)=1$ and $\|y\| \geqslant 1$. In fact, we will show that $\|y\|=1$. To prove this, let $G \in \mathcal{F}$.

1. If $i_{0} \in G$, then $\sum_{i \in G}\left|y_{i}\right| \leqslant \sum_{i \in G \cap F}\left|a_{i}\right|+\left|y\left(i_{0}\right)\right|$. However, $G \cap F \subsetneq F$, because otherwise we would have $F \cup\left\{i_{0}\right\} \in S_{\alpha}$. Thus,

$$
\sum_{i \in G}\left|y_{i}\right|<\sum_{i \in G \cap F}\left|a_{i}\right|+\left|y\left(i_{0}\right)\right|<1-\eta+\frac{\eta}{2}=1-\frac{\eta}{2} .
$$

2. If $i_{0} \notin G$, then $\sum_{i \in G}\left|y_{i}\right| \leqslant \sum_{i \in F}\left|a_{i}\right|=1$.

Hence, $\|y\| \leqslant 1$ which implies that $\|y\|=1$. However,

$$
g(y) \geqslant f(x)+g\left(e_{i_{0}}\right)=1+\frac{\eta}{2}
$$

which contradicts the fact that $\|g\|=1$. Therefore, $f \in \operatorname{ext}\left(B_{X_{\mathcal{F}}^{*}}\right)$.

Corollary 5.10. The dual of every combinatorial space has the CSRP.

Proof. It follows immediately from Corollary 5.8 and Theorem 5.9 .

\subsection{Polyhedrality of combinatorial spaces}

Fonf showed in [Fon81] that a polyhedral space must be $c_{0}$-saturated (that is, every infinite dimensional subspace has a further subspace isomorphic to $c_{0}$ ). In addition, for each countable $\alpha<\omega_{1}$ the space $X_{\mathcal{S}_{\alpha}}$ embeds isometrically in a $C(K)$ for an appropriately chosen countable compact Hausdorff space $K$ (see, for example, [CG91] or [Ros03]), which is polyhedral. Therefore each $X_{\mathcal{S}_{\alpha}}$ is a polyhedral Banach space.

In this section we will prove a stronger result: every combinatorial Banach space $X_{\mathcal{F}}$ is $(\mathrm{V})$-polyhedral. We will use the existence of an $\varepsilon$-gap for the sets in $\mathcal{F}$ that do not attain the norm of $x$ in $X_{\mathcal{F}}$, proved by Beanland, Duncan, Holt and Quigley:

Lemma 5.11 (BDHQ18, Lemma 2.5). Let $X_{\mathcal{F}}$ be a combinatorial space and let $x \in$ $S\left(X_{\mathcal{F}}\right)$. Then there exists an $\varepsilon_{x}>0$ so that

$$
\sum_{i \in F}\left|x_{i}\right|<1-\varepsilon_{x}
$$

for every $F \in \mathcal{F}$ such that $\sum_{i \in F}\left|x_{i}\right|<1$.

Theorem 5.12. Every combinatorial space $X_{\mathcal{F}}$ is (V)-polyhedral. 
Proof. Let $x \in S\left(X_{\mathcal{F}}\right)$ and $f \in \operatorname{ext}\left(B_{X_{\mathcal{F}}^{*}}\right)$ be such that $f(x)<1$. By Theorem 5.9, there exists $F \in \mathcal{F}^{\max }$ such that $f=\sum_{i \in F} \varepsilon_{i} e_{i}^{*}$, with $\varepsilon_{i} \in\{ \pm 1\}$ for every $i \in F$. Let $G=\left\{i \in F ; \varepsilon_{i}=\operatorname{sgn}\left(x_{i}\right)\right\}$ and $H=\left\{i \in F ; \varepsilon_{i}=-\operatorname{sgn}\left(x_{i}\right)\right\}$. Notice that

$$
\sum_{i \in G}\left|x_{i}\right| \neq 1
$$

Indeed, if $H=\emptyset$, then $f(x)=\sum_{i \in G}\left|x_{i}\right|<1$. On the other hand, if $H \neq \emptyset$, then

$$
\sum_{i \in G}\left|x_{i}\right|<\sum_{i \in F}\left|x_{i}\right| \leqslant\|x\|=1
$$

By Lemma 5.11, there exists $\varepsilon_{x}>0$ such that $\sum_{i \in G}\left|x_{i}\right| \leqslant 1-\varepsilon_{x}$. Hence,

$$
f(x)=\sum_{i \in G}\left|x_{i}\right|-\sum_{i \in H}\left|x_{i}\right| \leqslant 1-\varepsilon_{x}
$$

which proves the Theorem, since $\varepsilon_{x}$ depends only of $x$.

From Theorem 5.12 and Corollary 5.4 , it follows immediately:

Corollary 5.13. For every countable $\alpha, X_{\mathcal{S}_{\alpha}}$ is $(V)$-polyhedral and

$$
B_{X_{\mathcal{S}_{\alpha}}}=\overline{\mathrm{co}}\left(\operatorname{ext}\left(B_{X_{\mathcal{S}_{\alpha}}}\right)\right)
$$

In particular, $X_{\mathcal{S}_{\alpha}}$ is a solution to Lindenstrauss's problem.

\section{Questions and comments}

A new proof of Theorem 5.6 was given by Causey, using the fact that the extreme points of $C\left(K_{\mathcal{F}}\right)^{*}$, where $K_{\mathcal{F}}=\left\{\sigma \in\{-1,0,1\}^{\mathbb{N}}\right.$ : supp $\left.\sigma \in \mathcal{F}\right\}$, can be identified with $\left\{\varepsilon \delta_{\sigma}: \sigma \in K_{\mathcal{F}}, \varepsilon \in\{-1,1\}\right\}$. For this proof, see [ABC19, Proposition 4.1].

We cannot expect to obtain a (IV)-polyhedral space $X$ such that $B_{X}=$ $\overline{\mathrm{co}}\left(\operatorname{ext}\left(B_{X}\right)\right)$, since (IV)-polyhedral spaces do not have extreme points ([FV04, Theorem 3.6]). So, as the example provided by De Bernardi, our result is optimal. 



\section{Bibliography}

[AA92] Dale E. Alspach and Spiros A. Argyros. Complexity of weakly null sequences. Dissertationes Math. (Rozprawy Mat.), 321:44, 1992. URL: https://eudml org/doc/219311. 39

[ABC19] Leandro Antunes, Kevin Beanland, and Hùng Việt Chu. On the geometry of higher order Schreier spaces. arXiv e-prints, Mar 2019. Submitted. arXiv: 1903.03492, 21, 42, 74, 83

[AFGR17] Leandro Antunes, Valentin Ferenczi, Sophie Grivaux, and Christian Rosendal. Light groups of isomorphisms of Banach spaces and invariant LUR renormings. arXiv e-prints, November 2017. To appear in Pacific J. Math. arXiv:1711. 03482. 21, 37, 69

[AL87] Richard M. Aron and Robert H. Lohman. A geometric function determined by extreme points of the unit ball of a normed space. Pacific J. Math., 127(2):209231, 1987. URL: http://projecteuclid.org/euclid.pjm/1102699559. 73

[ALS91] Richard M. Aron, Robert H. Lohman, and Antonio Suárez. Rotundity, the C.S.R.P., and the $\lambda$-property in Banach spaces. Proc. Amer. Math. Soc., 111(1):151-155, 1991. doi:10.2307/2047873. 74

[Bae72] Albert Baernstein, II. On reflexivity and summability. Studia Math., 42:91-94, 1972. doi:10.4064/sm-42-1-91-94. 39

[Ban32] Stefan Banach. Théorie des opérations linéaires. Monografje Matematyczne. Warszawa, 1932. Tom I. 33, 42, 54, 66

[BDHQ18] Kevin Beanland, Noah Duncan, Michael Holt, and James Quigley. Extreme points for combinatorial Banach spaces. Glasgow Mathematical Journal, page 1-14, 2018. doi:10.1017/S0017089518000319, 40, 82

[Bea79] Bernard Beauzamy. Banach-Saks properties and spreading models. Math. Scand., 44(2):357-384, 1979. doi:10.7146/math.scand.a-11818. 39

[Bel86] Steven F. Bellenot. Banach spaces with trivial isometries. Israel J. Math., 56(1):89-96, 1986. doi:10.1007/BF02776242. 36, 59

[BL84] Bernard Beauzamy and Jean-Thierry Lapresté. Modèles étalés des espaces de Banach. Travaux en Cours. Hermann, Paris, 1984. URL: https://eudml. org/doc/273470. 39 
[Bou80] Jean Bourgain. Dentability and finite-dimensional decompositions. Studia Math., 67(2):135-148, 1980. doi:10.4064/sm-67-2-135-148. 27, 28

[BS30] Stefan Banach and Stanisław Saks. Sur la convergence forte dans le champ $L^{p}$. Studia Math., 2:51-57, 1930. URL: http://matwbn.icm.edu.pl/ksiazki/ or/or $2 /$ or $2111 . p d f .39$

[Cau17] Ryan M. Causey. Concerning the Szlenk index. Studia Math., 236(3):201-244, 2017. doi:10.4064/sm8197-10-2016. 40

[CG91] Jesús M. F. Castillo and Manuel González. An approach to Schreier's space. Extracta Math., 6(2-3):166-169, 1991. URL: https://eudml.org/doc/39945. 82

[Cha68] Srishti D. Chatterji. Martingale convergence and the Radon-Nikodym theorem in Banach spaces. Math. Scand., 22:21-41, 1968. doi:10.7146/math.scand a-10868. 28

[CS89] Peter G. Casazza and Thaddeus J. Shura. Tsirelson's space, volume 1363 of Lecture Notes in Mathematics. Springer-Verlag, Berlin, 1989. With an appendix by J. Baker, O. Slotterbeck and R. Aron. 47

[Day55] Mahlon M. Day. Strict convexity and smoothness of normed spaces. Trans. Amer. Math. Soc., 78:516-528, 1955. doi:10.2307/1993079, 22, 53

[DB17] Carlo Alberto De Bernardi. Extreme points in polyhedral Banach spaces. Israel J. Math., 220(2):547-557, 2017. doi:10.1007/s11856-017-1539-2.23. 72

[DGZ93] Robert Deville, Gilles Godefroy, and Václav Zizler. Smoothness and renormings in Banach spaces, volume 64 of Pitman Monographs and Surveys in Pure and Applied Mathematics. Longman Scientific \& Technical, Harlow; copublished in the United States with John Wiley \& Sons, Inc., New York, 1993. 49, 50, 51, 53, 54

[Die75] Joseph Diestel. Geometry of Banach spaces, volume 485 of Lecture Notes in Mathematics. Springer-Verlag, Berlin, 1975. 54

[DUJ77] Joseph Diestel and John Jerry Uhl Jr. Vector measures. Number 15 in Mathematical Surveys. American Mathematical Society, Providence, 1977. 28

[EW84] Gerald A. Edgar and Robert F. Wheeler. Topological properties of Banach spaces. Pacific J. Math., 115(2):317-350, 1984. URL: http://projecteuclid org/euclid.pjm/1102708251, 28, 32 
[FG10] Valentin Ferenczi and Elói Medina Galego. Countable groups of isometries on Banach spaces. Trans. Amer. Math. Soc., 362(8):4385-4431, 2010. doi: 10.1090/S0002-9947-10-05034-8, 22, 59

[FHH $\left.{ }^{+} 11\right]$ Marián Fabian, Petr Habala, Petr Hájek, Vicente Montesinos, and Václav Zizler. Banach space theory. CMS Books in Mathematics/Ouvrages de Mathématiques de la SMC. Springer, New York, 2011. doi:10.1007/ 978-1-4419-7515-7, 28, 63, 72, 75,

[FJ03] Richard J. Fleming and James E. Jamison. Isometries on Banach spaces: function spaces, volume 129 of Chapman $\&$ Hall/CRC Monographs and Surveys in Pure and Applied Mathematics. Chapman \& Hall/CRC, Boca Raton, FL, 2003. 33, 34, 35

[FLP01] Vladimir P. Fonf, Joram Lindenstrauss, and Robert R. Phelps. Infinite dimensional convexity. In William B. Johnson and Joram Lindenstrauss, editors, Handbook of the geometry of Banach spaces, pages 599-670, Amsterdam, 2001. North-Holland Publishing Co. v. 1. 27

[Fon81] Vladimir P. Fonf. Polyhedral Banach spaces. Mat. Zametki, 30(4):627634, 638, 1981. URL: https://link.springer.com/content/pdf/10.1007/ BF01137813.pdf. 82

[FR11] Valentin Ferenczi and Christian Rosendal. Displaying Polish groups on separable Banach spaces. Extracta Math., 26(2):195-233, 2011. 22, 52, 59, 64

[FR13] Valentin Ferenczi and Christian Rosendal. On isometry groups and maximal symmetry. Duke Math. J., 162(10):1771-1831, 2013. doi:10.1215/ 00127094-2322898. 67

[FV04] Vladimir P. Fonf and Libor Veselý. Infinite-dimensional polyhedrality. Canad. J. Math., 56(3):472-494, 2004. doi:10.4153/CJM-2004-022-7. 71, 72, 83

[GM14] Eli Glasner and Michael Megrelishvili. Representations of dynamical systems on Banach spaces. In Recent progress in general topology. III, pages 399-470. Atlantis Press, Paris, 2014. doi:10.2991/978-94-6239-024-9_9. 26

[Gow09] William T. Gowers. Must an "explicitly defined" Banach space contain $c_{0}$ or $\ell_{p}$ ?, Feb. 17, 2009. Gowers's Weblog: Mathematics related discussions. URL: https://gowers.wordpress.com/2009/02/17/ must-an-explicitly-defined-banach-space-contain-c_0-or-ell_p/. 40, 74 
[Hel80] Dietrich Helmer. Joint continuity of affine semigroup actions. Semigroup Forum, 21(2-3):153-165, 1980. doi:10.1007/BF02572546. 30

[Hol92] Melvin Randall Holmes. The universal separable metric space of Urysohn and isometric embeddings thereof in Banach spaces. Fund. Math., 140(3):199-223, 1992. URL: http://matwbn.icm.edu.pl/ksiazki/fm/fm140/fm14031.pdf. 37

[Jar88] Krzysztof Jarosz. Any Banach space has an equivalent norm with trivial isometries. Israel J. Math., 64(1):49-56, 1988. doi:10.1007/BF02767369. 36. 59

[JR85] John E. Jayne and Claude A. Rogers. Borel selectors for upper semicontinuous set-valued maps. Acta Math., 155(1-2):41-79, 1985. doi:10.1007/ BF02392537. 29.

[Kad59] Mikhail $\breve{I}$. Kadec'. Spaces isomorphic to a locally uniformly convex space. Izv. Vyš̌. Učebn. Zaved. Matematika, 1959(6 (13)):51-57, 1959. (In Russian). URL: http://testuvannya.com.ua/M. I.Kadets/PDF/10.pdf. 22, 51

[Kle60] Victor Klee. Polyhedral sections of convex bodies. Acta Math., 103:243-267, 1960. doi:10.1007/BF02546358. 71

[KPR84] Nigel J. Kalton, Newton T. Peck, and James W. Roberts. An F-space sampler, volume 89 of London Mathematical Society Lecture Note Series. Cambridge University Press, Cambridge, 1984. doi:10.1017/CB09780511662447. 37

[Lan93] Gilles Lancien. Dentability indices and locally uniformly convex renormings. Rocky Mountain J. Math., 23(2):635-647, 1993. doi:10.1216/rmjm/ 1181072581. 22, 51

[Lin66] Joram Lindenstrauss. Notes on Klee's paper: "Polyhedral sections of convex bodies". Israel J. Math., 4:235-242, 1966. doi:10.1007/BF02771638. 23, 72

[Loh89] Robert H. Lohman. The $\lambda$-function in Banach spaces. In Banach space theory (Iowa City, IA, 1987), volume 85 of Contemp. Math., pages 345-354. Amer. Math. Soc., Providence, RI, 1989. doi:10.1090/conm/085/983393. 74

[Meg98] Robert E. Megginson. An introduction to Banach space theory, volume 183 of Graduate Texts in Mathematics. Springer-Verlag, New York, 1998. doi: 10.1007/978-1-4612-0603-3. 25, 32

[Meg01] Michael G. Megrelishvili. Operator topologies and reflexive representability. In Nuclear groups and Lie groups (Madrid, 1999), volume 24 of Res. Exp. Math., pages 197-208. Heldermann, Lemgo, 2001. 21, 25, 26, 29, 30, 69 
[Meg03] Michael Megrelishvili. Fragmentability and representations of flows. In Proceedings of the 17th Summer Conference on Topology and its Applications, volume 27, pages 497-544, 2003. 53

[Nam67] Isaac Namioka. Neighborhoods of extreme points. Israel J. Math., 5:145-152, 1967. doi:10.1007/BF02771100. 27

[NW63] Togo Nishiura and Daniel Waterman. Reflexivity and summability. Studia Math., 23:53-57, 1963. doi:10.4064/sm-23-1-53-57. 39

[Pie84] Jean-Paul Pier. Amenable locally compact groups. Pure and Applied Mathematics (New York). John Wiley \& Sons, Inc., New York, 1984. A WileyInterscience Publication. 27

[Pie07] Albrecht Pietsch. History of Banach spaces and linear operators. Birkhäuser, Boston, 2007. 27

[Rai69] John Rainwater. Local uniform convexity of Day's norm on $c_{0}(\Gamma)$. Proc. Amer. Math. Soc., 22:335-339, 1969. doi:10.2307/2037051. 53

[Ros03] Haskell P. Rosenthal. The Banach spaces $C(K)$. In Handbook of the geometry of Banach spaces, Vol. 2, pages 1547-1602. North-Holland, Amsterdam, 2003. doi:10.1016/S1874-5849(03)80043-8. 82

[Rud87] Walter Rudin. Real and complex analysis. McGraw-Hill Book Co., New York, 3rd edition, 1987. 32

[Sch30] Józef Schreier. Ein gegenbeispiel zur theorie der schwachen konvergenz. Studia Math., 2:58-62, 1930. URL: https://eudml.org/doc/217264. 39

[ST90] Thaddeus J. Shura and David Trautman. The $\lambda$-property in Schreier's space $S$ and the Lorentz space $d(a, 1)$. Glasgow Math. J., 32(3):277-284, 1990. doi: 10.1017/S0017089500009368, 74

[Szl68] Wiesław Szlenk. The non-existence of a separable reflexive Banach space universal for all separable reflexive Banach spaces. Studia Math., 30:53-61, 1968. doi:10.4064/sm-30-1-53-61. 72 



\section{Index}

Convex polytope, 71

Dentability index, 51

Distinguished

family, 59

point, 59

Extension relation, 77

Extreme point, 42

Fragmented set, 29

Group

amenable, 27

bounded, 26

light, 26

orbit-wise light, 53

unitary, 26

Isometry group, 29

Maximal extension relation, 77

Maximal set, 40

Norm

$G$-invariant, 51

almost transitive, 67

equivalent, 50

locally uniformly convex (LUC), 49

locally uniformly rotund (LUR), 49

rotund, 49

strictly convex, 49

topology, 25

transitive, 67

Norming set, 75

Orbit, 67

Property

Banach-Saks property, 39 convex point of continuity property

(CPCP), 27

convex series representation property

(CSRP), 74

Kadec-Klee property (KKP), 50

point of continuity property (PCP),

27

Radon-Nikodym property (RNP), 28

uniform $\lambda$-property, 73

$\lambda$-property, 73

Regular family, 40

Renorming, 50

isometry invariant, 51

Schreier

family, 39

family of order $\alpha, 40$

set, 39

space, 39

spaces of higher order, 39

Space

combinatorial, 40

light, 29

polyhedral, 71

Spread, 41

Strong operator topology (SOT), 25

Support of a vector, 42

Unitary operator, 26

(V)-polyhedral space, 72

Weak operator topology (WOT), 25 